Florida International University FIU Digital Commons

$2-25-2008$

\title{
A Micro-Opto-Electro-Mechanical System (MOEMS) for Microstructure Manipulation
}

Jose Antonio Martinez

Florida International University, josemarteng@yahoo.com

DOI: $10.25148 /$ etd.FI10022533

Follow this and additional works at: https://digitalcommons.fiu.edu/etd

\section{Recommended Citation}

Martinez, Jose Antonio, "A Micro-Opto-Electro-Mechanical System (MOEMS) for Microstructure Manipulation" (2008). FIU Electronic Theses and Dissertations. 206.

https://digitalcommons.fiu.edu/etd/206 


\section{FLORIDA INTERNATIONAL UNIVERSITY}

Miami, Florida

\section{A MICRO-OPTO-ELECTRO-MECHANICAL SYSTEM (MOEMS) FOR MICROSTRUCTURE MANIPULATION}

A dissertation submitted in partial fulfillment of the

requirements for the degree of

DOCTOR OF PHILOSOPHY

in

ELECTRICAL ENGINEERING

by

Jose Antonio Martinez

2008 
To: Interim Dean Amir Mirmiran

College of Engineering and Computing

This dissertation, written by Jose Antonio Martinez, and entitled A Micro-Opto-ElectroMechanical System (MOEMS) for Microstructure Manipulation, having been approved in respect to style and intellectual content, is referred to you for judgment.

We have read this dissertation and recommend that it be approved.

Chunlei Wang

Grover Larkins, Jr.

Yuriy A. Vlassov

Roberto Panepucci, Major Professor

Date of Defense: February 25, 2008

The dissertation of Jose Antonio Martinez is approved.

Interim Dean Amir Mirmiran
College of Engineering and Computing

Florida International University, 2008 
(C) Copyright 2008 by Jose Antonio Martinez

All rights reserved. 


\section{DEDICATION}

To my wife Neelima, for all her love, patience and support. To my parents Nelly and Julio, for all their love, support, and constant encouragement. To my sisters Susana and Daniela, and my brother Jorge. Last but not least, to the memory of my dear papá Julio. 


\section{ACKNOWLEDGMENTS}

I would like to thank Dr. Roberto R. Panepucci for giving me the great opportunity to conduct this research under his guidance and support. I would also like to thank Dr. Chunlei Wang, Dr. Grover L. Larkins, Jr., and Dr. Yuriy A. Vlassov for being part of my committee, and for all their valuable collaboration.

I would like to acknowledge the Nanophotonics Research Lab team for all their support, technical discussions, and specially their friendship. Special thanks to Tao Liu for all his collaboration in this research work with his photonics expertise, and Patricia Fievre for all her valuable and kind help. Thanks also to Professor Anthony McGoron and Yuan Tang for providing the biological cells employed in this work as well as access to their lab's equipment, and to Professor Wei-Chiang Lin for letting me use his optical spectrum analyzer. Thanks to Neal Ricks for his training for the tools utilized in the Motorola Nanofabrication Research Facility.

This work was supported in part by the Air Force Office of Scientific Research (AFOSR) under grant FA9550-05-1-0232. 


\title{
ABSTRACT OF THE DISSERTATION \\ A MICRO-OPTO-ELECTRO-MECHANICAL SYSTEM (MOEMS) \\ FOR MICROSTRUCTURE MANIPULATION
}

\author{
by
}

Jose Antonio Martinez

Florida International University, 2008

Miami, Florida

\section{Professor Roberto Panepucci, Major Professor}

Microstructure manipulation is a fundamental process to the study of biology and medicine, as well as to advance micro- and nano-system applications. Manipulation of microstructures has been achieved through various microgripper devices developed recently, which lead to advances in micromachine assembly, and single cell manipulation, among others. Only two kinds of integrated feedback have been demonstrated so far, force sensing and optical binary feedback. As a result, the physical, mechanical, optical, and chemical information about the microstructure under study must be extracted from macroscopic instrumentation, such as confocal fluorescence microscopy and Raman spectroscopy.

In this research work, novel Micro-Opto-Electro-Mechanical-System (MOEMS) microgrippers are presented. These devices utilize flexible optical waveguides as gripping arms, which provide the physical means for grasping a microobject, while simultaneously enabling light to be delivered and collected. This unique capability allows extensive optical characterization of the structure being held such as transmission, reflection, or fluorescence. The microgrippers require external actuation which was 
accomplished by two methods: initially with a micrometer screw, and later with a piezoelectric actuator. Thanks to a novel actuation mechanism, the "fishbone", the gripping facets remain parallel within 1 degree. The design, simulation, fabrication, and characterization are systematically presented. The devices mechanical operation was verified by means of 3D finite element analysis simulations. Also, the optical performance and losses were simulated by the 3D-to-2D effective index (finite difference time domain FDTD) method as well as 3D Beam Propagation Method (3D-BPM). The microgrippers were designed to manipulate structures from submicron dimensions up to approximately $100 \mu \mathrm{m}$. The devices were implemented in SU-8 due to its suitable optical and mechanical properties. This work demonstrates two practical applications: the manipulation of single SKOV-3 human ovarian carcinoma cells, and the detection and identification of microparts tagged with a fluorescent "barcode" implemented with quantum dots. The novel devices presented open up new possibilities in the field of micromanipulation at the microscale, scalable to the nano-domain. 


\section{TABLE OF CONTENTS}

CHAPTER

PAGE

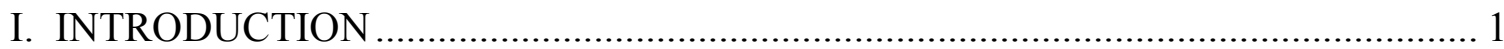

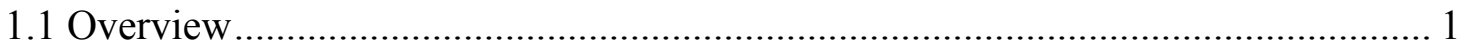

1.2 Current work

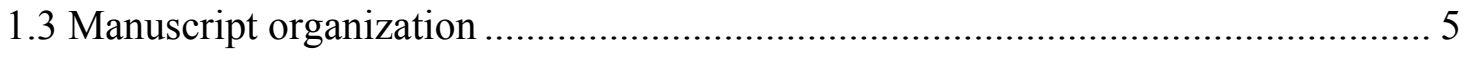

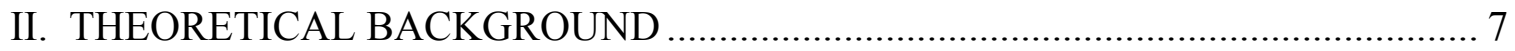

2.1 Micro-Electromechanical Systems (MEMS) ………...................................... 7

2.1.1 Fabrication techniques and issues .......................................................... 8

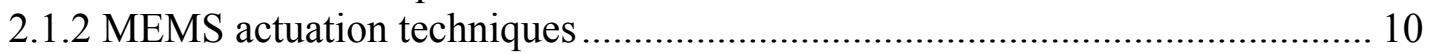

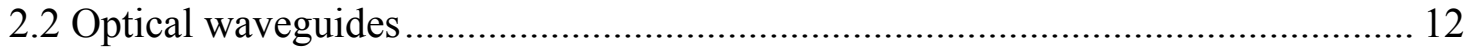

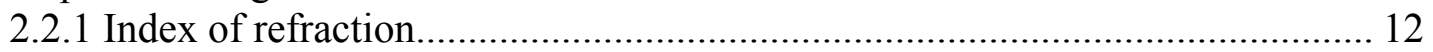

2.2.2 Snell's law and total internal reflection (TIR) .............................................. 13

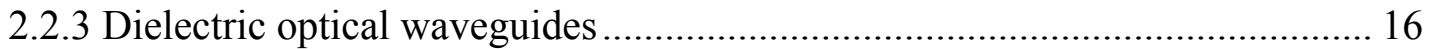

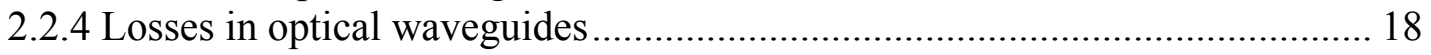

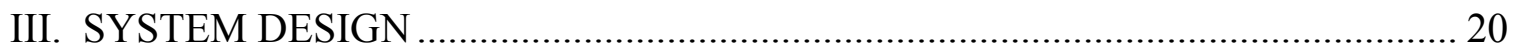

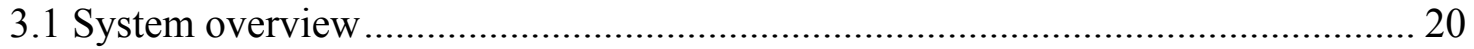

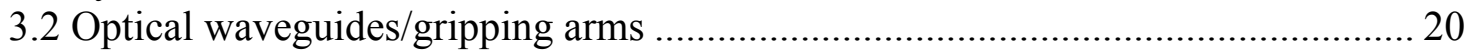

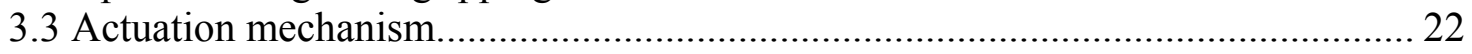

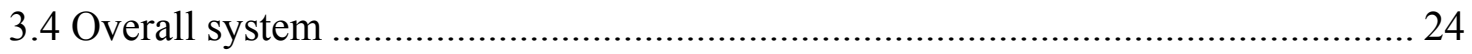

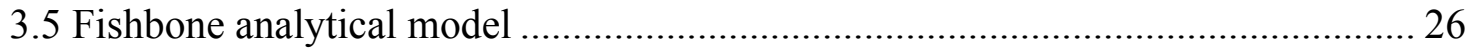

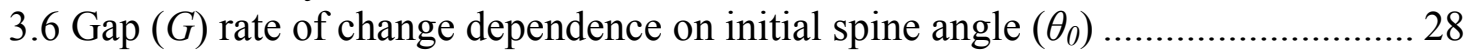

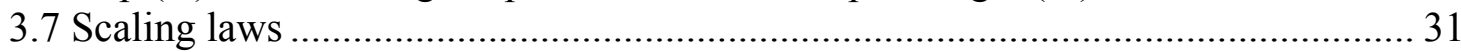

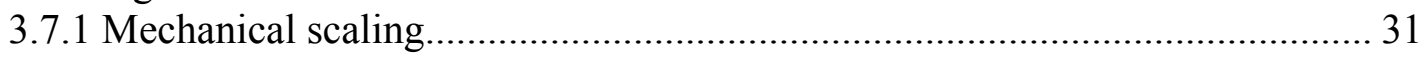

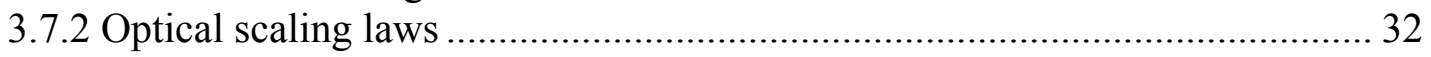

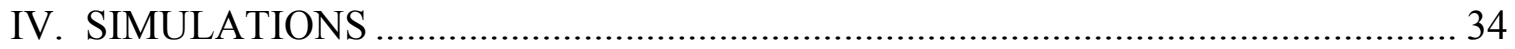

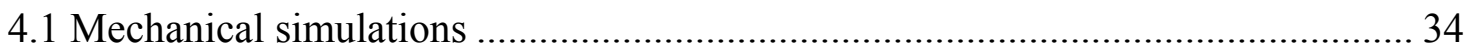

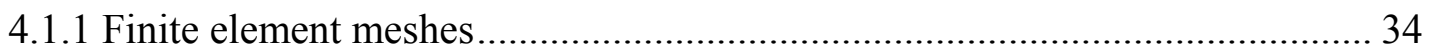

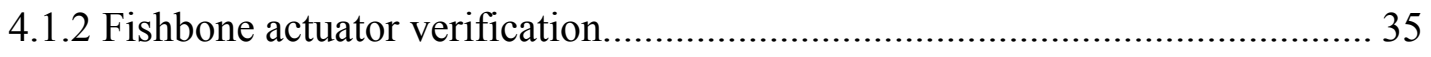

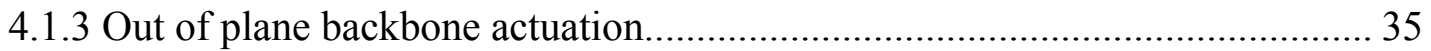

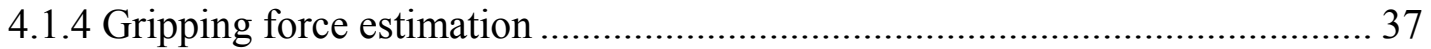

4.2 Optical waveguides/Gripping arms simulation..................................................... 38

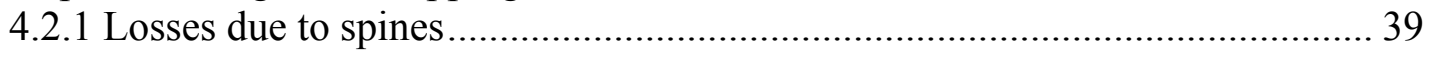

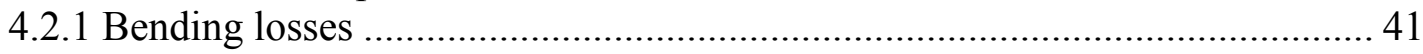

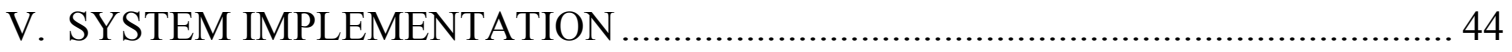

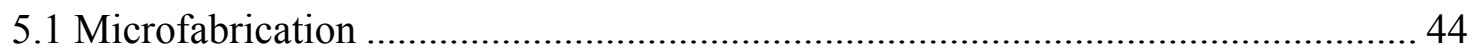

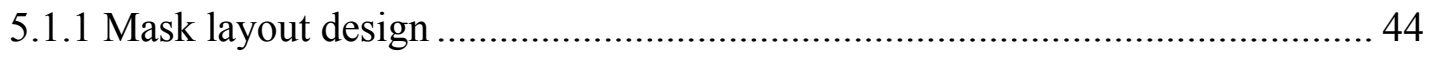

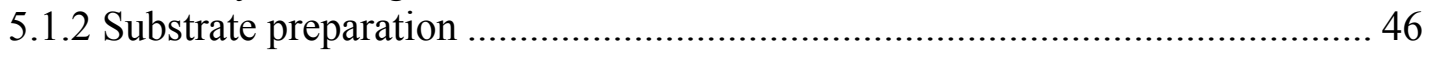

5.1.3 Photolithography ………………………………...................................... 47 


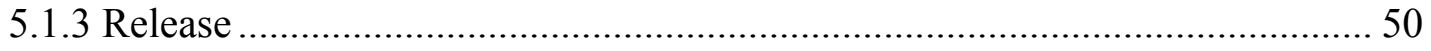

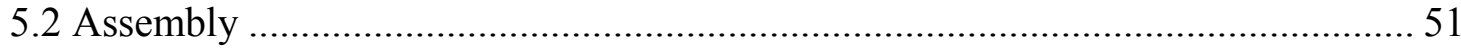

VI. MECHANICAL CHARACTERIZATION AND RESULTS ……………...............55

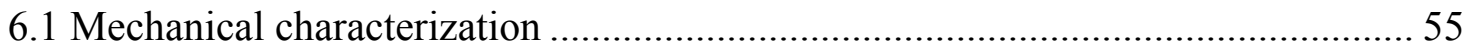

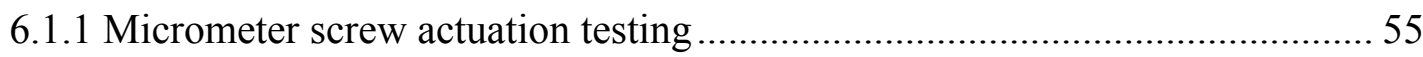

6.1.2 Piezoelectric actuation testing.................................................................... 58

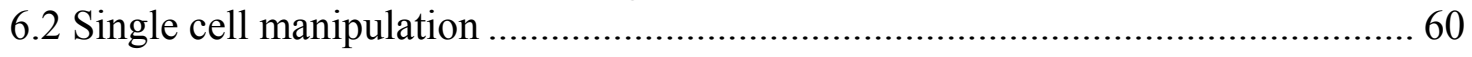

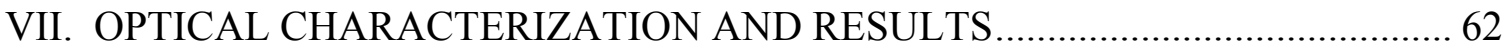

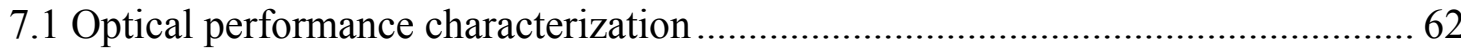

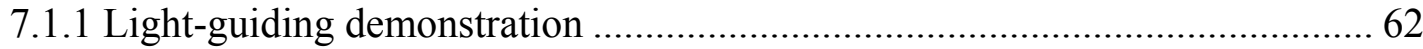

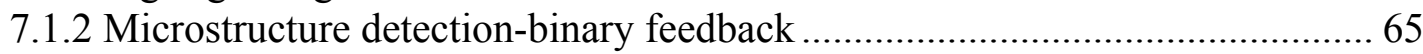

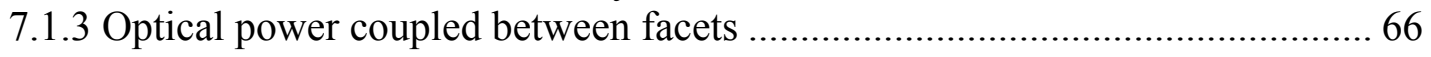

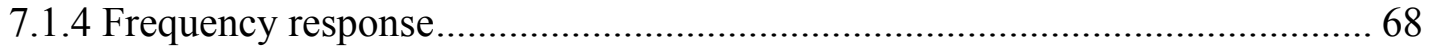

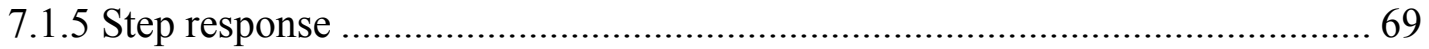

7.2 Quantum dot tagged micro parts identification .................................................. 70

VIII. SUMMARY, CONCLUSIONS, AND FUTURE WORK ……………………........ 74

8.1 Summary and conclusions ........................................................................... 74

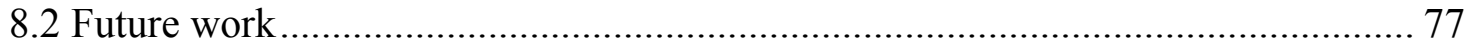

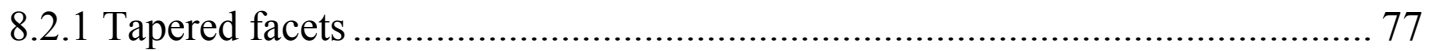

8.2.2 Fluorescence detection optimization........................................................... 78

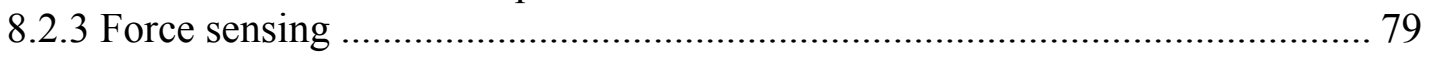

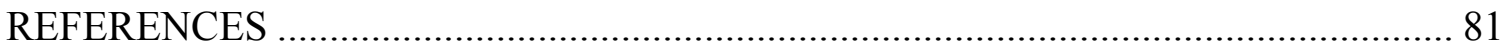

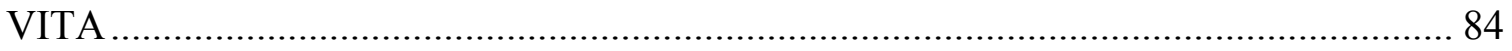




\section{LIST OF FIGURES}

FIGURE

PAGE

2.1 Wet release sequence utilized in surface micromachining. After the sacrificial material has been etched and rinsed by a given liquid, the device is dried. Bottom left: a successfully released freestanding structure; bottom right: collapsed structure due to stiction. 10

2.2 When a ray arrives to a boundary between two mediums, it is reflected at an angle $\theta_{r}$ equal to the incident angle $\theta_{i}$. The portion of the beam that passes across the boundary is deflected to make an angle $\theta_{t}$ with respect to the normal (also called refracted).

2.3 Three cases of a light beam traveling from glass (higher index) to air (lower index): a) the beam is incident at a smaller angle than $\theta_{c}$ which causes some of the light to be reflected, and some refracted or transmitted, b) the beam is incident at $\theta_{c}$ which prevents any light to be refracted, and c) the beam is incident at an angle larger than $\theta_{c}$, so total internal

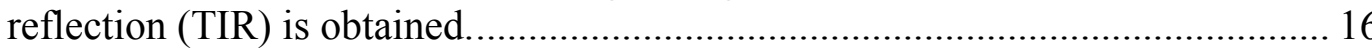

2.4 Two rays entering a dielectric waveguide. Ray $A$ enters at an angle $\alpha>\alpha_{\max }$ which causes $\theta<\theta_{c}$ at the cladding, and consequently allowing ray $A$ to be refracted into the cladding and eventually to escape the waveguide. Ray $B$ enters the waveguide at an angle $\alpha<\alpha_{\max }$ which causes $\theta>\theta_{c}$ at the cladding, causing total internal reflection (modified from [24]).

3.1 Conceptual diagram of MOEMS microgrippers. The fundamental elements are the flexible waveguides which also perform as gripping arms, and the actuation mechanism in charge of controlling the separation between the gripping arms to grasp a microobject.

3.2 Fishbone actuation mechanism for MOEMS microgrippers. a) relaxed structure, b) gripping arms closing by pulling the backbone (- $x$ direction), and c) gripping arms opening by pushing the backbone $(+x$ direction). Notice that the gripping arms remain parallel and their motion is limited to only the $\pm y$ direction.

3.3 MOEMS microgripper showing its different components. Notice that the gripping elements are also optical waveguides so not only do they provide the mechanical means to manipulate microstructures, but also carry light to and from the microstructure to be manipulated.

3.4 Analytical modeling of fishbone mechanism. a) microgripper with relaxed fishbone showing the initial gap $g_{0}$, initial spine angle $\theta_{0}$, and spine length 
$L$. b) Geometrical representation of gap variation $\Delta g$ by moving the backbone by $\Delta S$.

3.5 Theoretical gap range vs. shaft displacement dependence on initial spine angle $\theta_{0}$ predicted by equation 3.5. Curves are plotted up to the maximum theoretical gap value.

3.6 Relation between the gap and the displacement induced by the shaft for a fixed gripping arm-backbone separation, and spines at $22.5^{\circ}, 45^{\circ}$, and $67.5^{\circ}$. Notice that higher angles provide smaller rates of change, and therefore more control over the gap dimensions.

4.1 Meshes generated by COMSOL Multiphysics, used for finite element based simulation corresponding to the 50 (left) and $100 \mu \mathrm{m}$ (right) microgrippers. The meshes are composed of 13951 and 11720 elements respectively.

4.2 3D Simulated microgrippers actuation (birds eye view) for an $8 \mu \mathrm{m}$ backbone pull closing the grippers and opened to $\sim 54 \mu \mathrm{m}$ after a $20 \mu \mathrm{m}$ backbone push. On the left: $50 \mu \mathrm{m}$ (top) and $100 \mu \mathrm{m}$ (bottom) gripper closed. On the right: $50 \mu \mathrm{m}$ (top) and $100 \mu \mathrm{m}$ (bottom) gripper opened. Color scale represents levels of Von Mises stress post actuation. The lower limit of the scale (blue) represents minimum levels while top (red) represents maximum levels 36

4.3 $50 \mu \mathrm{m}$ arm microgripper effective spring constant determination through simulations. Pulling the backbone beyond the microgripper closing point produces reaction forces between the facets, by which spring-like behavior is observed. a) backbone pulled $30 \mu \mathrm{m}$ beyond closure, b) Reaction forces at the facet vs. backbone additional displacement.

4.4 Staircase like transmission due to the four $20 \mu \mathrm{m}$ spines attached to the output waveguide, for microgrippers waveguide widths of 10, 20,30, 40, 50 , and $100 \mu \mathrm{m}$. Notice that the losses introduced on the $10 \mu \mathrm{m}$ waveguide are significant, and therefore it goes rapidly out of the graph scale.

4.5 Light propagating along the waveguide bends corresponding to the 10, 20, and $30 \mu \mathrm{m}$ waveguides bent at a radius of $270 \mu \mathrm{m}$. The input light was distributed among the first 10 modes (fundamental plus modes 1 through 9) of each waveguide with TE polarization.

4.6 Continuation from previous figure. Light propagating along the waveguide bends corresponding to the 40,50 , and $100 \mu \mathrm{m}$ waveguides bends. 
5.1 Layout of frame supporting 12 microgrippers of 10, 20, 30, 40, 50, and $100 \mu \mathrm{m}$ gripping arm width, two of each, designed to be utilized for mechanical testing only. Notice that the microgripper arms are flush with the backbone.

5.2 Layout of frame supporting microgrippers in their final design form, with elongated gripping arms to be bonded to optical fibers. The microgrippers are attached to a large frame by means of thin stubs strong enough to hold each microgripper in place during microfabrication, but weak enough to break once the microgripper has been bonded to the hollow cylinder, actuator and optical fibers.

5.3 Fabrication process steps: 1: Silicon substrate with $2 \mu \mathrm{m}$ of $\mathrm{SiO}_{2}, 2$ : SU-8 Spin-coat, 3: UV exposure, 4: Develop, 5: Complete structure release in BOE. 48

5.4 Exposure matrix results for SU-8 at $30 \mu \mathrm{m}$ thickness, monitoring the profile of the $10 \mu \mathrm{m}$ arm microgripper, since it has the smallest critical dimension. $8-24$ seconds exposures corresponding to $100-300 \mathrm{~mJ} / \mathrm{cm}^{2}$ doses. The best profile was observed with the 24 second dose.

5.5 (a) SEM micrograph of released main frame supporting MOEMS microgrippers, (b) Close-up detail of $30 \mu \mathrm{m}$ arm width microgripper supported by 4 stubs.

5.6 a) Completion of microgripper assembly for mechanical operationcharacterization only. Notice that there are no optical fibers present. b) Microgripper diagram showing its different components

5.7 Piezoelectric actuator/microgripper assembled system. The arrow points to the microgripper

5.8 Assembly process sequence. 1) Shaft bonding to backbone, 2) first fiber alignment-bonding process, 3) second fiber is bonded with the same procedure, 4) Microgripper is detached from the main frame.

6.1 Microgripper (50 $\mu \mathrm{m}$ arm) actuated mechanically with a micrometer screw showing different levels of actuation. (a) $2.4 \mu \mathrm{m}$, (b) $5 \mu \mathrm{m}$, (c) $10 \mu \mathrm{m}$, (d) $20 \mu \mathrm{m}$, (e) $30 \mu \mathrm{m}$, and (f) $40 \mu \mathrm{m}$.

6.2 Microgripper (50 $\mu \mathrm{m}$ arm) actuated mechanically with a micrometer screw. a) Actuated to $\sim 130 \mu \mathrm{m}, \mathrm{b}$ ) Device failure when attempted to open to 140 $\mu \mathrm{m}$, fractures occurred.

6.3 Microcrack present at the "V" junctions pointed by white arrow. Even though the device is expected to fracture at these points under extensive 
actuation, device failure (fracture) could happen prematurely at these points due to microcrack propagation

6.4 Measured microgripper gap (100 $\mu \mathrm{m}$ arm) for different voltages. Voltage was increased in steps of 10 volts from 0 to 120 volts and then lowered back to 0 volts as indicated by the arrows showing hysteresis. After some time, the gap returns to the starting value.

6.5 Manipulation of a single SKOV-3 cell utilizing a $50 \mu \mathrm{m}$ wide arm microgripper. 1) microgripper closed, 2) gripper opens, 3) cell is approached and grasped, 4) cell is moved and isolated.

7.1 System diagram showing setup for MOEMS microgripper testing. Laser light is coupled to the microgripper by means of an optical fiber. The light coupled to through the facets is monitored by means of a photo detector or a spectrometer.

7.2 Coupling light from a $128 \mathrm{~mW}, 532 \mathrm{~nm}$ laser to the input fiber. Output power was monitored by connecting the output fiber to a photo-detector. 1a) SMF28 fiber with approximately $10 \mu \mathrm{m}$ core was used to couple light into the $100 \mu \mathrm{m}$ waveguide arm of the microgripper. 2a) $50 \mu \mathrm{m}$ multimode fiber was used to couple light into a $50 \mu \mathrm{m}$ waveguide arm, allowing a better matching of modes and uniform illumination. Figures $1 \mathrm{~b}$ and $2 \mathrm{~b}$ are $1 \mathrm{a}$ and $1 \mathrm{~b}$ under a filter that eliminates $532 \mathrm{~nm}$, showing the fluorescence of the SU-8 material

7.3 Comparison between simulated and observed light propagation at the $100 \mu \mathrm{m}$ waveguide bends: a) Simulation from launching the first 10 modes, b) Experimental observation of modes reflecting at the polymer-air interface, notice ray-like propagation which resembles the simulation.

7.4 SEM Micrograph showing a section of SU-8 optical waveguide. The top smoothness comes from spin-coating the polymer, while the side roughness is due to the photolithographic fabrication process which chemically defines the sidewalls.

7.5 Microobject detection with binary feedback. a) the microgripper with nothing between the facets, where light is coupled from the left to the right arm, and b) the microgrippers grasping a $20 \mu \mathrm{m}$ thick aluminum slab. Notice the significant reduction of light in the left arm, with corresponding increase in illumination at the input arm do to the high reflectivity of the slab.

7.6 Optical power monitored at the output of the microgripper's opening is increased from its initial state by $\sim 5 \mu \mathrm{m}$. 
7.7 Calculated transmitted power at a wavelength of $532 \mathrm{~nm}$ for a SU-8 - air SU- 8 cavity as the cavity length is increased by $5 \mu \mathrm{m}$.

7.8 Frequency response from $50 \mu \mathrm{m}$ arm microgripper measured by monitoring the coupled light modulation induced by varying the gap between the facets (opening and closing the microgripper). The $-3 \mathrm{~dB}$ frequency is approximately $500 \mathrm{~Hz}$.

7.9 Normalized curves corresponding to the power coupled across the facets in response to steps of different magnitudes. The time it takes for the optical power coupled to drop from $10 \%$ to $90 \%$ was measured to be 1.6 $\mathrm{ms}$ for the 30 Volts step (incrementing the gap by $2 \mu \mathrm{m}$ ) and $2.5 \mathrm{~ms}$ for the 120 volts step (incrementing the gap by $14 \mu \mathrm{m}$ ).

7.10 Micropart identification through fluorescence detection. a) Microgripper approaching a QD tagged SU-8 slab with (laser off), b) Microgripper grasping the SU-8 slab, collecting the emitted fluorescence.

7.11 Fluorescence detected from SU-8 microparts tagged with QDs with emission at 555, 599, and $612 \mathrm{~nm}$ with an integration time of $500 \mathrm{~ms}$.

7.12 Fluorescence detected from a single SU-8 micropart tagged with QDs with emission at $599 \mathrm{~nm}$ with integration times ranging from $50 \mathrm{~ms}$ up to $1000 \mathrm{~ms}$. Notice that even at the shortest integration times, there is still a high signal to noise ratio.

8.1 Conceptual diagram of a $50 \mu \mathrm{m}$ arm microgripper with tapered gripping arms to produce $10 \mu \mathrm{m}$ wide facets.

8.2 Conceptual diagram showing a modified facet to include a curved metallic aperture which would optimize the fluorescence coupled to the receiving facet.

8.3 Conceptual diagram implementation of force sensing. A Bragg grating is patterned on one of the microgripper's arms. A circulator is utilized to couple the laser required for the microgripper operation, and a tunable source for force sensing. 


\section{INTRODUCTION}

\subsection{Overview}

Humans have the ability to manipulate objects because of the presence of hands, particularly fingers and muscles. Besides grasping an object, humans are able to extract information such as texture, rigidity, temperature, as well as color and shape from it, being this amount of information directly related to the human senses, or feedback. However, as objects become smaller, in order to accomplish manipulation, humans have need of different tools (i.e. tweezers), and, as the objects enter the microscopic level, more sophisticated tools or instruments are necessary. In order to accomplish accurate manipulation, these tools require different types of feedback. The development of these more sophisticated tools is the foundation for the field of micromanipulation.

Micromanipulation has been an area with significant interest by researchers. In general, the goal has been to produce a system that would allow precise and controlled manipulation of structures of different kinds in the micrometer range. With this goal in mind, different Micro-Electro-Mechanical Systems (MEMS) microgrippers have been developed, with applications such microobject manipulation [1], microelectronic devices manipulation [2], assembly of biomedical microdevices [3], single biological cell manipulation [4-7], and micro-assembly of 3-D MEMS structures [8].

Micro-Electro-Mechanical systems (MEMS) microgrippers developed by other researchers have been fabricated using different materials, with different actuation techniques, as well as feedback mechanisms. A material used very often for the implementation of microgrippers is SU-8, which was used, for example, for the 
microgrippers developed by Chronis [5], Honnaty [4], and Nguyen [7], implemented with thermal actuators. Also fabricated in SU-8 are the microgripper presented by Choi [1] which is piezoelectrically actuated, and the one presented by Roch [2] which uses a shape memory alloy actuator. It should be mentioned that, even though the aforementioned microgrippers have demonstrated significant advances in the field of micromanipulation, none of them is equipped with any kind of integrated feedback. More sophisticated, or instrumented microgrippers, have been implemented in silicon, such as the microgripper presented by Beyeler [6] which is equipped with a monolithically fabricated force feedback sensor and is actuated electrostatically by means of a comb actuator, or the hybrid type microgripper presented by Park [9] which is implemented in silicon, actuated piezoelectrically and is equipped with a force sensor. Microgrippers have also been implemented in metal such as the one presented by Carrozza [3] which is implemented in nickel and is instrumented with a semiconductor strain-gauge force sensor. Microgrippers reported by $\mathrm{Lu}[10]$ are implemented in silicon dioxide, electrothermally actuated, and equipped with an optical sensor fabricated underneath the gripping facets. This sensor provides the information of whether a micro-object is present between the facets or not; however, this requires the presence of the substrate containing the sensor in the proximity of the gripping facets, limiting significantly the micromanipulation capabilities of the device.

It is clear that the more feedback mechanisms a microgripper can have, the more accurate its micromanipulation ability will be. Furthermore, different feedback mechanisms can provide different types of information about the microobject being manipulated. The types of feedback implemented in microgrippers are force sensing, 
implemented in silicon [6,9] and nickel [3], and a binary optical feedback implemented in $\mathrm{SiO}_{2}$ through a CMOS process [10]. So, because of the limited feedback implemented so far, there is still physical, mechanical, optical and chemical information about the microstructure under study which requires the use of macroscopic instrumentation, such as confocal fluorescence microscopy, Raman spectroscopy, nanoindentation, among others [11].

\subsection{Current work}

In this work, the development of novel Micro-Opto-Electro-Mechanical System (MOEMS) microgrippers is presented. Flexible optical waveguides are utilized as gripping arms, which not only provide the physical means for grasping a microobject, but also enable light to be delivered and also collected in transmission, reflection or fluorescent mode, allowing extensive optical characterization of the structure being held. Furthermore, inherently, the microgrippers are capable of providing binary feedback to determine if there is a microstructure or not between the gripping arms. The simultaneous application of these functions introduces a new set of possible applications of microgrippers beyond micro manipulation, such as optical fluorescence measurements of biological entities, micro-part identification, and several mechano-functional studies in biology and medicine. An intriguing possibility is that the integrated optical properties could even enable optical feedback where vision and electric feedback methods are not possible. This could be accomplished by optical recognition of an optical fluorescent barcode held by the microgripper. To the best of the author's knowledge, this is the first microgripper implemented in SU-8 with a feedback mechanism. 
The MOEMS microgrippers presented require external actuation which was accomplished by two different methods; initially with a micrometer screw, and later with a piezoelectric actuator, working in conjunction with a novel mechanical structure named "fishbone" due to its shape. This external actuation approach is enabled by the "fishbone" structure which controls the separation between the gripping arms. To the best knowledge of the author the fishbone structure is a unique design, and a first of its kind for microelectro-mechanical-system (MEMS) devices, or Micro-Opto-Electro-Mechanical Systems (MOEMS); its key advantages over other approaches will be discussed in later chapters. The external actuator, along with the fishbone structure eliminate the need of any electrical signal in the vicinity of the microgripper, and due to the mechanical nature of the actuation technique, there is absolutely no increase in temperature at the gripping facets, which significantly increases the biocompatibility of the device. The microgrippers presented in this work are implemented with gripping elements (optical waveguides) widths of 10, 20, 30, 40,50 and $100 \mu \mathrm{m}$. Experimental results are focused on the $50 \mu \mathrm{m}$ and $100 \mu \mathrm{m}$ grippers which were easily assembled and tested, and scaling laws are presented to predict performance into the submicron domain. The overall microgrippers size is $1200 \mu \mathrm{m}$ by $500 \mu \mathrm{m}$; it should be mentioned that the overall dimensions of these microgrippers increases the feasibility of a macroassembly technique of the gripper-micromanipulator-light source system, while smaller gripping elements allow the manipulation of structures with dimensions ranging from submicron, up to $100 \mu \mathrm{m}$. The material of choice for the MOEMS microgrippers fabrication was SU-8, a negative tone, epoxy based, high aspect ratio photoresist. As mentioned before, SU-8 is highly suitable for MEMS fabrication [12], has been widely used for implementation of 
microgrippers, and has also been demonstrated suitable for the production of low loss polymer optical waveguides [13]. Based on the aforementioned facts, SU-8 is perfectly suitable for the implementation of the MOEMS microgrippers. It should be mentioned that SU-8 is highly transparent for wavelengths longer than $360 \mathrm{~nm}$ [14].

In this work, two practical applications are demonstrated: the precise manipulation of single SKOV-3 human ovarian carcinoma cells with size in the range of $10 \mu \mathrm{m}$ to $30 \mu \mathrm{m}$, and the detection and identification of microparts tagged with a fluorescent "barcode" implemented with quantum dots, with emission wavelengths of $555 \mathrm{~nm}, 599 \mathrm{~nm}, 612 \mathrm{~nm}$. This work represents the first implementation of a functional Micro-Opto-ElectroMechanical (MOEMS) microgripper with integrated optical waveguides.

\subsection{Manuscript organization}

This manuscript is divided in eight chapters including the present one. Chapter II provides the necessary background for understanding the theory behind the two main fields involved in this work, and the advantages of combining them, MicroElectromechanical Systems, and optical waveguides, to produce Micro-Opto-ElectroMechanical Systems (MOEMS). Chapter III presents the complete device design process, followed by chapter IV which presents mechanical and optical simulations, and discusses the optical and mechanical scaling laws for the devices under study. Chapter V describes all the steps involved in the surface micromachining process to produce the optical microgrippers, which includes the thin film processing, along with the assembly required to connect the microgripper to the external actuator and optical fibers. Chapters VI and VII present the mechanical and optical characterization respectively of the $50 \mu \mathrm{m}$ and 
$100 \mu \mathrm{m}$ arm microgrippers along with demonstration of practical applications. Chapter VIII presents a summary and conclusions of the research performed, along with suggested future work for the further development of the MOEMS microgrippers. The manuscript ends with a list of references cited, and the author's vita. 


\section{THEORETICAL BACKGROUND}

\subsection{Micro-Electromechanical Systems (MEMS)}

Micro-Electromechanical systems (MEMS) have been under research for more than four decades, but the simple concept behind them remains the same. MEMS are devices and machines which operate mechanically on a microscopic level, and are achieved by the integration of mechanical elements, and actuators, typically on a common substrate.

Historically, the MEMS field of research was originated in December 1959 by Richard P. Feynman with a talk given at the annual meeting of the American Physical Society named "There is Plenty of Room at the bottom" [15]. The main point of this talk was that, for him, the laws of physics posed no limit to miniaturization of systems, and that the only limitation to miniaturization was our ability to make physically smaller things. Feynman investigated the miniaturization of different things that did not require a particular size such as numbers, information storage, and computing, but ended investigating the miniaturization of machinery [16]. His first concrete example of a micromachine was an electric motor which he presented in [17]. A significant factor for the evolution of the field of MEMS was the integrated circuit industry, which uses photographic and chemical etching techniques to "print" small circuits on a substrate. The roots of this evolution took place specially from the mid 1960s to 1980s, when for example, sparse attempts of developing systems of this kind took place, such as to sculpt three dimensional features in a planar silicon substrate by anisotropic etching [18]. Up to now, many practical MEMS devices have been developed and are commercially available such as micro-nozzles used in inkjet cartridges, accelerometers for airbag deployment, 
micromirror arrays for digital light modulation, electromechanical switches, as well as the core MEMS of this research, microgrippers.

\subsubsection{Fabrication techniques and issues}

When considering the extension of integrated circuit (IC) fabrication techniques to micromechanical structures, one fundamental difference is that the IC fabrication domain is essentially two dimensional and for MEMS fabrication, three dimensional sculpting techniques are necessary. In contrast with conventional IC fabrication, the fabrication of MEMS structures, which have unsupported regions, is concerned with mechanical material properties such as Young's modulus, temperature coefficient of expansion, or yield strength [19]. Over the years different fabrication techniques for mechanical structures based on IC manufacturing processes such as bulk and surface micromachining have been developed. Bulk micromachining is based on creating mechanical structures in the silicon substrate bulk by selectively combining highly anisotropic (orientation dependent) etchants with isotropic (non-directional) etchants, and the wafer's crystallographic orientation $[16,20]$. Surface micromachining is based on patterning thin film layers of material deposited on a substrate or wafer; in this technique, wherever an open area or a free standing structure is needed, a thin film material is deposited and is called sacrificial material, or also known as spacer. The material out of which the free standing structure is made is called structural material $[16,20]$. The fabrication process is finished by removing the sacrificial material, leaving a free standing structure. A third fabrication technique worth mentioning is LIGA processing, which in German stands for Lithographie Galvanoformung Abformung (lithographic 
electrodeposition molding). This technique is capable of producing high aspect ratio structures by first creating a mold of the desired structure on the surface of a conductive substrate out of thick x-ray photoresist; this mold is filled and covered with metal by electroplating; the metal structure is then separated from the mold, and can be used repeatedly as a mold insert for injection molding [20,21].

The fabrication yield of MEMS by means of surface micromachining is mainly limited by stiction, a phenomenon in which freestanding structures tend to stick to the substrate or nearby system components. After the structural material has been deposited and patterned on top of the sacrificial material, a release process takes place, in which the sacrificial material is removed to leave a free standing structure. Removal of the sacrificial layer is usually accomplished by wet etching, however, as the liquid etchant removes the sacrificial layer and fills the space between the structure and the substrate, the dominant force is surface tension. Usually, the etchant is rinsed with a liquid, which is then dried. Some times, as the etchant liquid is dried, surface tension forces pull down the structural material, until it makes contact with the substrate, resulting in a nonoperational device [19] (see figure 2.1). One possible solution to overcome stiction is super critical drying technique, in which the etchant is rinsed with a liquid (usually $\mathrm{CO}_{2}$ ) which is then driven into a supercritical phase under high pressure state, at which the liquid does not present surface tension, increasing significantly the probability of obtaining a freestanding structure [21]. Another alternative to overcome stiction is to follow a dry release approach, for example, a $\mathrm{SiO}_{2}$ sacrificial layer can be removed with anhydrous hydrofluoric acid vapor, or a polymer sacrificial layer can be removed with oxygen plasma. 


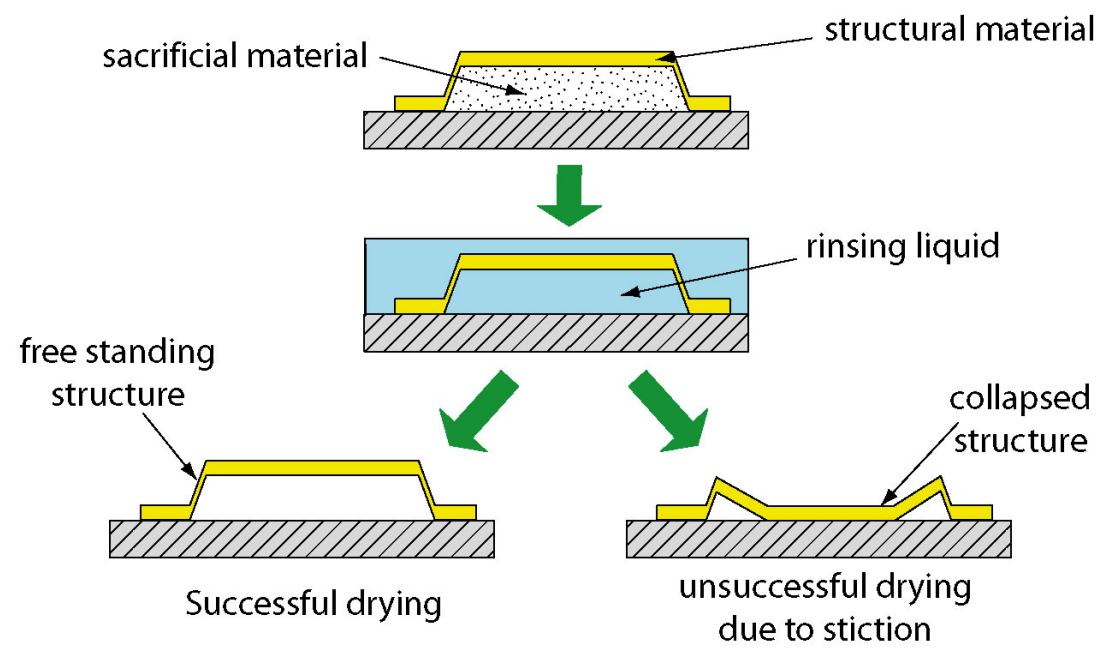

Figure 2.1. Wet release sequence utilized in surface micromachining. After the sacrificial material has been etched and rinsed by a given liquid, the device is dried. Bottom left: a successfully released freestanding structure; bottom right: collapsed structure due to stiction.

As it will become evident in later chapters, the devices developed in this work are produced by means of a surface micromachining fabrication process in which the sacrificial layer is present underneath the complete structure, and not only in specific areas as it is done in conventional MEMS. Upon fabrication completion, instead of having mechanical structures attached to a substrate, the microgrippers in a supporting frame are completely released (detached) from the substrate. So, due to the release procedure nature for the presented microgrippers, stiction does not represent an obstacle for successful fabrication. More details will be provided in Chapter V, which describes in detail the fabrication process.

\subsubsection{MEMS actuation techniques}

In the field of MEMS, actuation refers to the act of effecting or transmitting mechanical motion, forces, and work by a device or system on its surroundings in response to the application of a bias voltage or current, converting one type of energy into 
another $[16,21]$. Throughout the development of this field, different actuation methods have been developed and can be classified according to the type of physical stimulus that causes the actuation, being the most common stimuli electric fields, magnetic fields, and thermal effects. Commonly used actuators include electrostatic, piezoelectric, electromagnetic, thermal, and shape memory alloy [19]. It should be mentioned that depending on the application, some actuators are more suitable than others, depending on the required displacement, how fast of a response is needed, and even if a linear or nonlinear response with respect to the input is required. Different actuation mechanisms are summarized in table 2.1 .

A popular technique of actuation used in microgrippers developed by other researchers has been thermal actuation, which relies on thermal expansion in response to localized heating $[4,5,7]$. Other actuation techniques employed also in microgrippers have been electrostatic [6], piezoelectric [1,9], and shape memory alloy [2]. The microgrippers presented in this work require external actuation, which was accomplished by two different methods: initially with a micrometer screw, and later with a piezoelectric actuator commercially available, in both cases connected to the microgrippers through a shaft. Actuation with the micrometer screw is simply accomplished by turning its knob to displace the shaft. Piezoelectric actuation is accomplished by applying a voltage to a piezoelectric element, which generates a strain linearly proportional to the electric field generated by the voltage [19], expanding the element in size, displacing the shaft. A drawback of piezoelectric actuation is that piezoelectric materials inherently exhibit nonlinear hysteresis, specially when operated in a relatively long range [22, 23], nevertheless, this can be overcome by feedback from strain sensors, in a way that any 
nonlinearity due to hysteresis could be compensated. Another possibility to overcome this drawback is to employ a nonlinear hysteresis model to estimate hysteresis effects and compensating this effects using feed-forward control schemes [23].

Table 2.1. Actuation methods for MEMS summary (from [19])

\begin{tabular}{|c|c|c|c|}
\hline $\begin{array}{c}\text { Actuation } \\
\text { Mechanism }\end{array}$ & Displacement & Response time & $\begin{array}{l}\text { Input/ } \\
\text { output }\end{array}$ \\
\hline Electrostatic & microns & tens of microseconds & nonlinear \\
\hline Piezoelectric & microns & tens of microseconds & linear \\
\hline Magnetostatic & tens of microns & $\begin{array}{l}\text { Hundreds of } \\
\text { microseconds }\end{array}$ & nonlinear \\
\hline Thermal & microns & tens of milliseconds & nonlinear \\
\hline $\begin{array}{l}\text { Shape } \\
\text { memory }\end{array}$ & 100 s of microns & tens of milliseconds & nonlinear \\
\hline
\end{tabular}

\subsection{Optical waveguides}

From a general point of view, an optical waveguide is a medium that is capable of guiding light, or in other words, electromagnetic waves at frequencies corresponding to the optical spectrum. For the purpose of providing the necessary background to understand the optical functionality of the microgrippers presented in this work, it is necessary to describe some fundamental knowledge of optics and more precisely optical waveguides. For this, some basic concepts will be presented in the following subsections.

\subsubsection{Index of refraction}

The index of refraction $n$ of a material can be defined as the ratio of the speed of light traveling in free space to the speed of light traveling in the material (phase velocity $v$ ), as presented in equation 2.1 .

$$
n=\frac{c}{v}
$$


For a light wave traveling in a dielectric material, given that the material is nonmagnetic $\left(\mu_{\mathrm{r}}=1\right)$, the phase velocity can be expressed in terms of the relative permittivity $\varepsilon_{r}$, the absolute permittivity $\varepsilon_{0}$ and absolute permeability $\mu_{0}$ as:

$$
v=\frac{1}{\sqrt{\varepsilon_{r} \varepsilon_{0} \mu_{0}}}
$$

Also, the speed of light can be expressed as:

$$
c=\frac{1}{\sqrt{\varepsilon_{0} \mu_{0}}}=3 \times 10^{8} \frac{\mathrm{m}}{\mathrm{s}}
$$

Thus, combining 2.2 and 2.3, the index of refraction of a dielectric, nonmagnetic material can be expressed in terms of its relative permittivity as:

$$
n=\sqrt{\varepsilon_{r}}
$$

From equation 2.2 it can be seen that light travels at a slower speed than the speed of light in a medium with higher relative permittivity than free space $\left(\varepsilon_{r}=1\right)$. From equation 2.4 it can be seen that that medium will have a higher index of refraction $(n>1)$ than free space. It is important to keep in mind that both $\varepsilon_{r}$, and consequently $n$, are material parameters which depend on frequency. A list of some approximate indexes of refraction for some common materials, including SU-8 is presented in table 2.2 [24].

\subsubsection{Snell's law and total internal reflection (TIR)}

To understand how it is that a medium is capable of confining light, it is convenient to analyze the case of a ray of light traveling in a medium with index of refraction $n_{1}$, and incident to the boundary the medium forms with a second medium with index of refraction $n_{2}$, from a geometrical optics point of view. When the ray arrives to 
Table 2.2. Index of refraction for some common materials.

\begin{tabular}{lc}
\hline Material & $\begin{array}{c}\text { Index of } \\
\text { Refraction }\end{array}$ \\
\hline Air & 1.00 \\
Water & 1.33 \\
Ethyl alcohol & 1.36 \\
Fused silica & 1.46 \\
Silica glass & 1.50 \\
Polystyrene & 1.59 \\
SU-8 & 1.60 \\
Silicon & 3.50 \\
\hline
\end{tabular}

the boundary at a given angle of incidence $\theta_{i}$, it is reflected at and angle $\theta_{r}$ equal to the angle of incidence. Both angles measured with respect to the normal to the boundary, and with values between $0^{\circ}$ and $90^{\circ}$. Furthermore, if a fraction of the ray crosses the boundary, the transmitted ray (also called refracted) will propagate in the second medium at an angle $\theta_{t}$, (see figure 2.2) determined from Snell's law which states the relation between the indexes of refraction of the two mediums and the transmitted and incidence angles. Snell's law [25] is presented in equation 2.5.

$$
n_{1} \cdot \sin \left(\theta_{i}\right)=n_{2} \cdot \sin \left(\theta_{t}\right)
$$

Which can also be expressed as:

$$
\frac{n_{1}}{n_{2}}=\frac{\sin \left(\theta_{t}\right)}{\sin \left(\theta_{i}\right)}
$$

From equation 2.6 it can be seen that when light propagates from a medium with higher index of refraction to a medium with lower index, $\theta_{t}$ will be greater than $\theta_{i}$, and when propagating from lower to higher index mediums, $\theta_{t}$ will be smaller than $\theta_{i}$. As a rule of thumb, light will always be deflected towards the higher index medium. "When light traveling in a certain medium is reflected off an optically denser material (one with a 


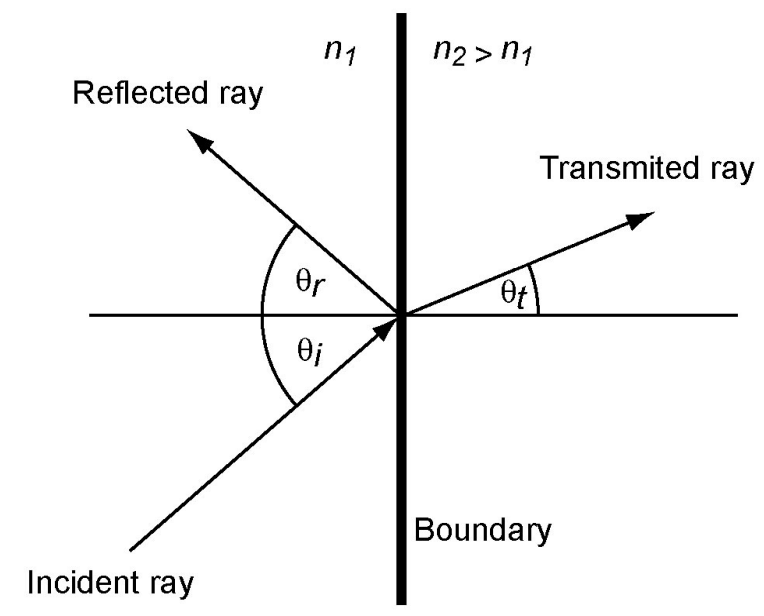

Figure 2.2. When a ray arrives to a boundary between two mediums, it is reflected at an angle $\theta_{r}$ equal to the incident angle $\theta_{i}$. The portion of the beam that passes across the boundary is deflected to make an angle $\theta_{t}$ with respect to the normal (also called refracted).

higher refractive index), the process is referred to as external reflection. Conversely, the reflection of light off of less optically dense material (such as light traveling in a glass being deflected at a glass-to-air interface) is called internal reflection" [26].

Consider the case of light traveling in glass, incident to a boundary with air (figure 2.3a). As the angle of incidence $\theta_{i}$ increases, $\theta_{t}$ increases accordingly, until reaching $90^{\circ}$ (figure $2.3 \mathrm{~b}$ ); at this point no light propagates anymore into the second medium. The incidence angle at which light stops propagating in the lower index material is called critical angle $\theta_{c}$ and can be calculated from equation 2.7, which is derived by making $\theta_{t}=90^{\circ}$ in equation 2.6.

$$
\theta_{c}=\sin ^{-1}\left(\frac{n_{2}}{n_{1}}\right)
$$

Increasing the angle of incidence beyond $\theta_{c}$ causes total internal reflection (TIR), which means that ideally all the incident light will be reflected back into the higher index medium (figure 2.3c), at an angle equal to the angle of incidence. 


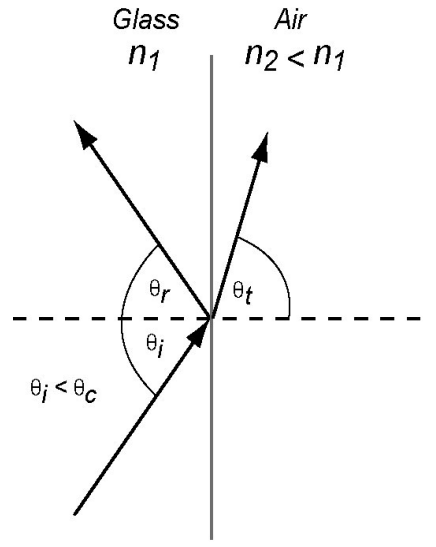

(a)

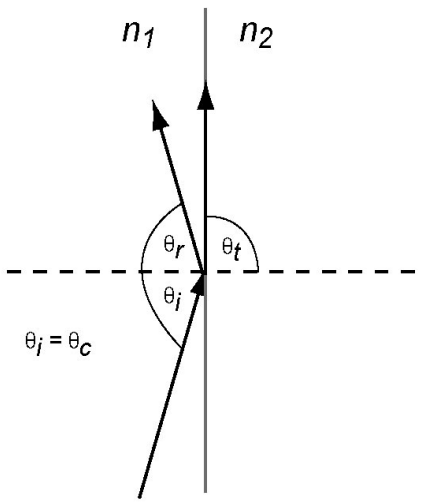

(b)

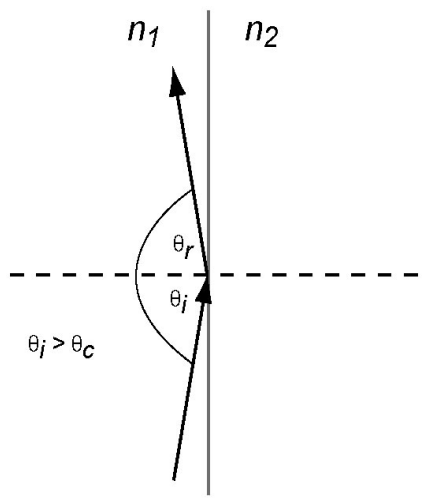

(c)

Figure 2.3. Three cases of a light beam traveling from glass (higher index) to air (lower index): a) the beam is incident at a smaller angle than $\theta_{c}$ which causes some of the light to be reflected, and some refracted or transmitted, b) the beam is incident at $\theta_{c}$ which prevents any light to be refracted, and c) the beam is incident at an angle larger than $\theta_{c}$, so total internal reflection (TIR) is obtained.

\subsubsection{Dielectric optical waveguides}

A dielectric waveguide is composed of a dielectric material (core) surrounded by a second dielectric material (cladding) with lower index of refraction. This structure is capable of confining electromagnetic energy in the form of light, and it guides it in the direction of its axis of symmetry. The ability of a waveguide to collect light incident onto its input facet is determined by its numerical aperture $(N A)$. In analogy to the $N A$ of a lens, it is an indication of the half angle of light that is 'focused' into the guided modes. From Snell's law, and the total internal reflection previously discussed, the following relation is obtained:

$$
n_{0} \sin \left(\alpha_{\max }\right)=n_{1} \sin \left(\theta_{c}\right)=\sqrt{n_{1}^{2}-n_{2}^{2}}
$$

Where $n_{0}$ is the index of refraction of the medium in front of the facet, $n_{l}$ is the index of refraction of the core, $n_{2}$ is the refractive index of the cladding, and $\alpha_{\max }$ is the maximum acceptance angle. The angle of the cone formed by the maximum acceptance angle is 
given by $2 \alpha_{\max }$ and it is referred to as total acceptance angle. The numerical aperture $N A$ of the waveguide can be expressed as:

$$
N A=n_{0} \cdot \sin \left(\alpha_{\max }\right)=\sqrt{n_{1}^{2}-n_{2}^{2}}
$$

The previous discussion can be illustrated with figure 2.4, which depicts two rays entering a dielectric waveguide. Ray $A$ does not propagate along the waveguide, and ray $B$ does propagate along the axis of symmetry of the waveguide. Ray $A$ arrives to the facet at an incident angle greater than $\alpha_{\max }$, which causes its transmitted fraction to travel inside the core of the waveguide for a short distance, until it reaches the cladding at a smaller angle than $\theta_{c}$, not sufficient for TIR, and consequently propagating into the cladding and finally escaping the waveguide. Ray $B$ arrives to the facet at an incident angle smaller than $\alpha_{\max }$, which causes its transmitted fraction to travel inside the core, and to arrive to the cladding at an angle greater than $\theta_{c}$, sufficient to cause TIR, and therefore reflecting the ray back towards the center of the waveguide, and repeating the process every time the cladding is met.

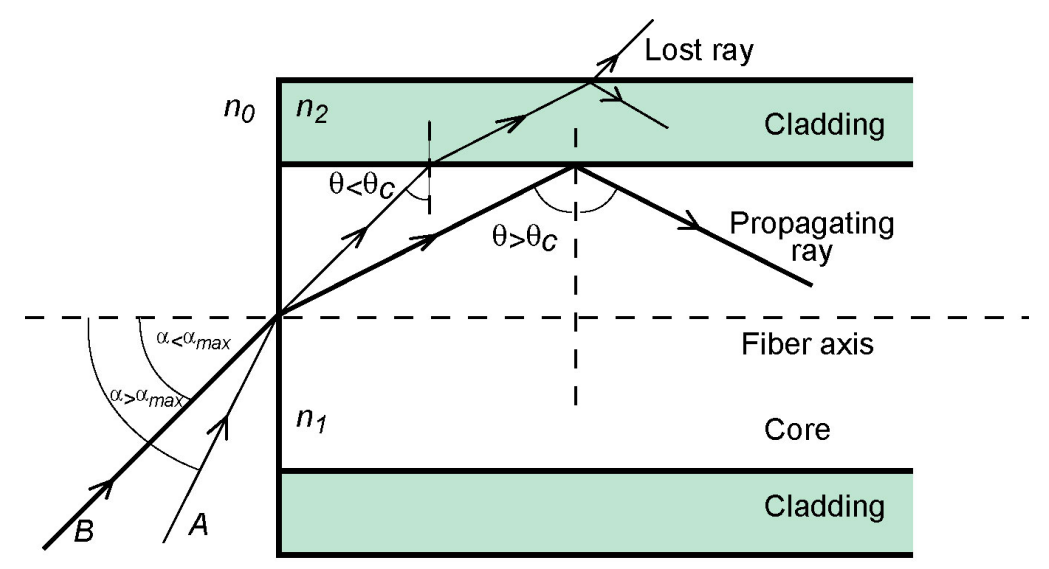

Figure 2.4. Two rays entering a dielectric waveguide. Ray $A$ enters at an angle $\alpha>\alpha_{\max }$ which causes $\theta<\theta_{c}$ at the cladding, and consequently allowing ray $A$ to be refracted into the cladding and eventually to escape the waveguide. Ray $B$ enters the waveguide at an angle $\alpha<\alpha_{\max }$ which causes $\theta>\theta_{c}$ at the cladding, causing total internal reflection (modified from [24]). 
It is also relevant to describe the reflectance and transmittance of normally incident light to a boundary. Reflectance R quantifies the intensity of light reflected with respect to that of the normal incident light, and can be calculated from equation 2.10.

$$
R=\left(\frac{n_{1}-n_{2}}{n_{1}+n_{2}}\right)^{2}
$$

Transmittance $T$ quantifies the intensity of light transmitted with respect to that of the normal incident light, and can be calculated with equation 2.11. Equations 2.10 and 2.11 are both valid for internal and external reflection. Intuitively, the portion of transmitted light and the portion of reflected light must add to unity [24].

$$
T=\frac{4 n_{1} n_{2}}{\left(n_{1}+n_{2}\right)^{2}}
$$

\subsubsection{Losses in optical waveguides}

As mentioned before, the waveguides implemented in this work are fabricated in SU-8, an epoxy like polymer. When dealing with polymer optical waveguides, there are different types of losses present. The losses can be classified as input and output coupling, bending, and propagation losses. Input and output losses are present due to the non ideal coupling between fibers and the waveguides; bending losses take place when the waveguide changes direction or shape since bends host leaky modes instead of perfectly bound modes; finally, propagation losses are present due to material losses and waveguide sidewall roughness, which is highly dependent on the fabrication technique of choice [27]. The microgripper flexible optical waveguides have additional propagation losses introduced by the fishbone actuation mechanism, given that the spines are physically attached to the waveguides. In the case of the microgrippers waveguides, the 
losses that require special attention are the bending losses, and the losses introduced by the spines. Bending losses require attention because of the relatively small bending radius required to implement gripping facets with the waveguides. Spine losses are specific to the microgrippers, and therefore require special attention as well. Bending losses present on the waveguides, as well as losses introduced by the spines will be estimated through simulations in Chapter IV. 


\section{SYSTEM DESIGN}

\subsection{System overview}

To implement a microgripper capable of carrying light to and from a microobject to be manipulated, there are two requirements: a medium to carry the light to and from the microobject to be held, and a mechanical structure that would allow manipulation. The MOEMS microgrippers presented in this work have two fundamental components which suffice the previously mentioned requirements. The fundamental components are: flexible optical waveguides which at the same time perform as gripping arms, and the actuation mechanism in charge of flexing the waveguides and consequently opening and closing the microgripper. Figure 3.1 portrays a conceptual diagram which indicates the arrangement of fundamental components discussed above.

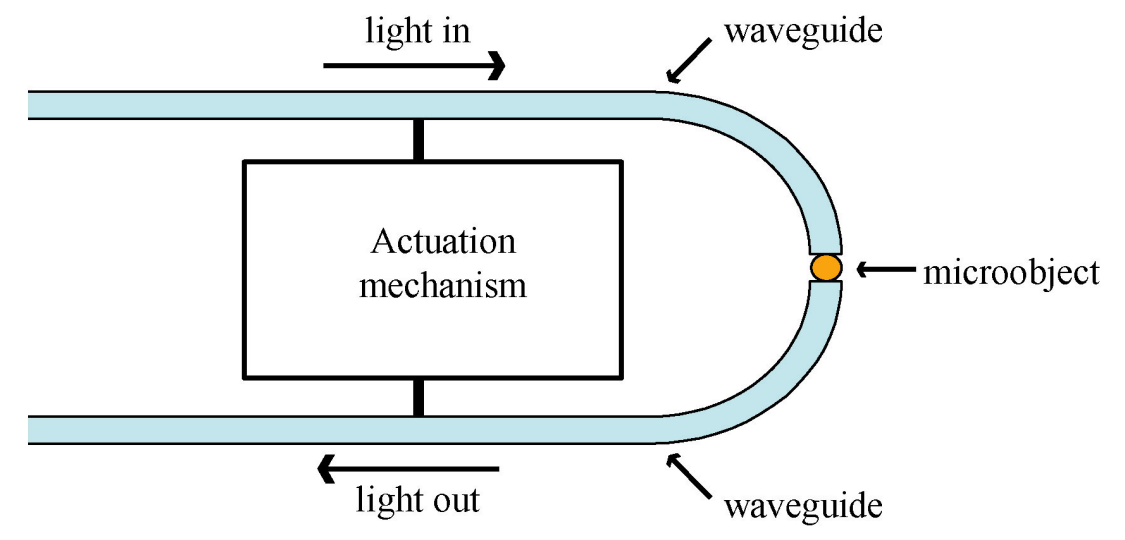

Figure 3.1. Conceptual diagram of MOEMS microgrippers. The fundamental elements are the flexible waveguides which also perform as gripping arms, and the actuation mechanism in charge of controlling the separation between the gripping arms to grasp a microobject.

\subsection{Optical waveguides/gripping arms}

The gripping arms are designed to not only be able to grasp microobjects, but also to perform as flexible optical waveguides so they are capable of carrying light to and from the microstructure being held. The range of microobject sizes that can be manipulated 
with the microgrippers depends on the dimensions of the gripping facets, which at the same are the cross section of the optical waveguides. In order to cover a range of microobjects sizes ranging from submicron to $100 \mu \mathrm{m}$ in diameter, the microgrippers are designed to have gripping arms ranging from $10 \mu \mathrm{m}$ up to $100 \mu \mathrm{m}$ wide, given that different gripping arms/optical waveguides widths are suitable for manipulation of different microstructure sizes. It is evident that the most suitable microgripper for manipulation of submicron structures would be the one with the narrowest gripping arms, provided that a high magnification microscope is used to visually control the process. The thickness of the microgripper arms is defined during the fabrication process by the spin coating speed of SU-8, which was fixed at $30 \mu \mathrm{m}$, producing the smallest waveguide cross section/gripping facet of $10 \mu \mathrm{m} \times 30 \mu \mathrm{m}$ and the largest of $100 \mu \mathrm{m} \times 30 \mu \mathrm{m}$.

The wave guiding properties of the structure are dependent on parameters such as the waveguide width, height, and index of refraction, and also on sidewall characteristics, which are a strongly dependent on the fabrication technique. SU-8 optical waveguides have been demonstrated previously [13], with losses in the range of $0.19 \pm 0.03 \mathrm{~dB} / \mathrm{cm}$ at $638.8 \mathrm{~nm}$ (red) for waveguides with cross-sections of $5 \mu \mathrm{m} \times 5 \mu \mathrm{m}$; such low loss waveguides were fabricated by direct proton beam writing technique, and consequently produced smooth sidewalls. A significant difference between the previously mentioned SU-8 optical waveguides is the cladding; the microgripper optical waveguides do not have a solid cladding, instead, they are surrounded by air. Since the index of refraction of SU-8 $(n=1.6[13])$ is greater that that of air $(n=1.0)$, it is possible to obtain total internal reflection and consequently, light guiding condition is still satisfied (refer back to figure 2.4). 
The ability of the receiving waveguide to collect light incident onto its input facet is determined by the numerical aperture $(N A)$ of the waveguide. Recalling from Chapter II, the numerical aperture of the waveguide can be expressed as:

$$
N A=n_{0} \cdot \sin \left(\alpha_{\max }\right)=\sqrt{n_{1}^{2}-n_{2}^{2}}
$$

Where $n_{0}$ is the index of refraction of the medium in front of the facet, $n_{l}$ is the index of refraction of the core, $n_{2}$ is the refractive index of the cladding, and $\alpha_{\max }$ is the maximum acceptance angle. The case of a SU-8 waveguide core with air cladding leads to the ideal situation of total acceptance angle of $2 \alpha_{\max }=180^{\circ}$, which means that all light incident in the facet is coupled into a guided mode, due to total internal reflection at the sidewalls. It is worth mentioning that, inside liquid media such as water with $n=n_{2}=1.33$, the acceptance angle reduces to $2 \alpha_{\max }=109^{\circ}$, which is still significantly large.

\subsection{Actuation mechanism}

In order to open and close the gripping arms, an actuation mechanism is required (refer to figure 3.1). This mechanism could be implemented in several ways; however, there are certain desirable aspects that need to be considered. First, a symmetrical actuation mechanism, which will induce an identical but opposite displacement upon the gripping arms is desired. Keeping the center of the space between the gripping facets fixed facilitates the positioning of the microgripper with respect to the object to be manipulated; this can be contrasted with an asymmetrical actuation mechanism such as the one presented by Beyeler in [6] in which one of the gripping arms is pushed by a lateral comb actuator until the gripping arms are closed, point at which the gripping force

deflects the second arm. Second, maintaining the parallelism between the gripping facets 
within a given range of separation facilitates the manipulation of different microobject sizes with the same microgripper; this could be compared to an adjustable wrench, which keeps its jaw elements parallel in order to be useful for different nut sizes. A structure that suffices the previously discussed requirements, which the author named "fishbone" because of its shape, is presented in figure 3.2a. The fishbone structure is composed of spines, and a backbone, located in the center of the structure; at the same time, the complete fishbone structure is located between the gripping arms, attached by the ends of the spines.

The fishbone principle of actuation is based on inducing controlled push and pull displacements of the center of the fishbone structure located between the gripping arms, namely the backbone. Figure 3.2 presents a diagram of the fishbone actuation mechanism indicating its parts, named with the terminology followed in this work, and two cases of

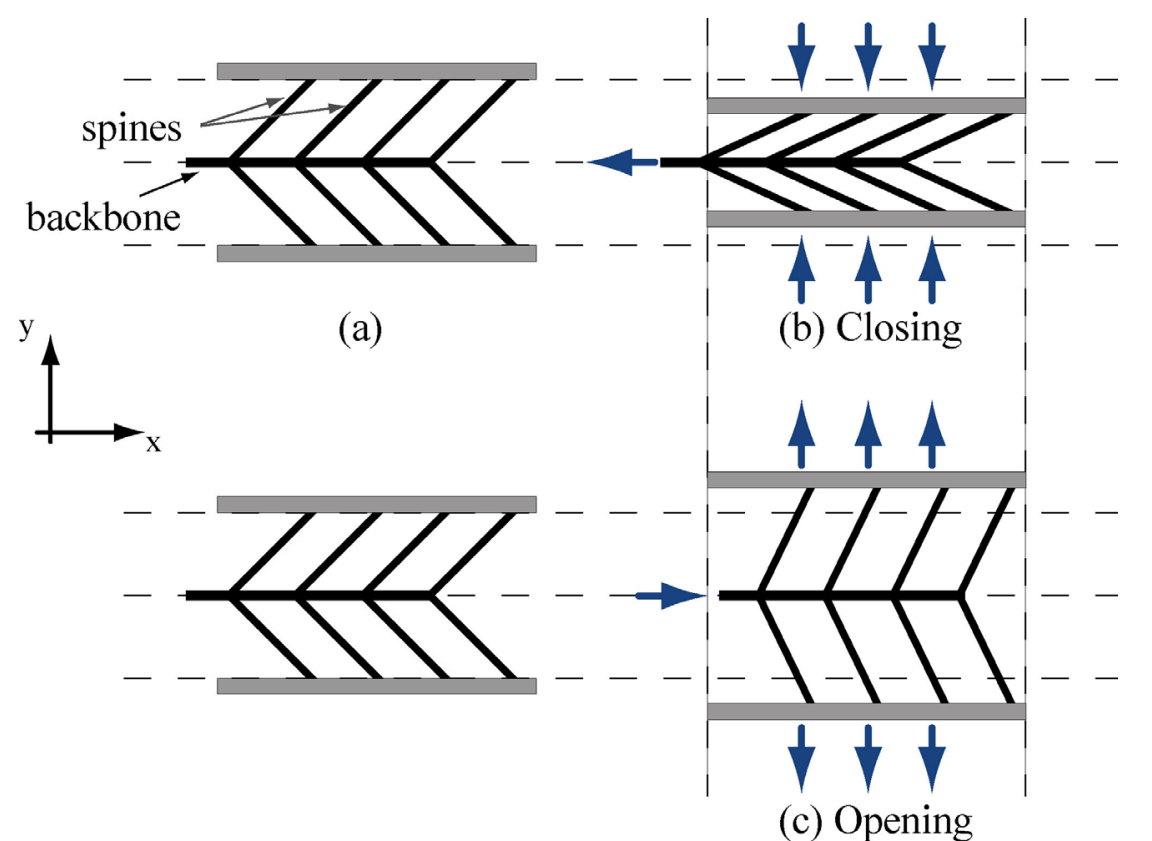

Figure 3.2. Fishbone actuation mechanism for MOEMS microgrippers. a) relaxed structure, b) gripping arms closing by pulling the backbone ( $-x$ direction), and c) gripping arms opening by pushing the backbone $(+x$ direction $)$. Notice that the gripping arms remain parallel and their motion is limited to only the $\pm y$ direction. 
actuation. When the backbone of the fishbone structure is pulled (-x direction), the spines experience tensile stresses, consequently pulling the gripping elements together, bringing the gripper arms closer to each other, and therefore closing the gripper (figure $3.2 \mathrm{~b}$ ). In a similar manner, when the backbone is pushed forward $(+x$ direction), the spines experience compressive forces, pushing the gripping elements apart, and therefore causing the gripper to open (figure 3.2c). The fact that the gripping elements are pushed/pulled apart by four identical pairs of spines that receive the same exact displacement turns to be highly beneficial to the performance of the microgripper because the gripping facets remain parallel at all times, being this a very attractive feature of this unique design. It should be mentioned that this feature allows the manipulation of different microstructure sizes within a wide range, while at all times the gripping facets remain parallel. The degree of parallelism will be estimated from simulations in the following chapter.

\subsection{Overall system}

In order to induce a controlled motion on the backbone, an actuator is attached to it by means of a shaft bonded with UV curable glue. As explained previously, the opening and closing of the gripper is achieved by relative motion between the backbone and the gripping elements; for this the bases of the microgripper arms need to be anchored while the rest of the structure is free to move. The gripping arms are designed to be bonded to the outside wall of a hollow cylinder. In order to couple and collect light to and from the microgripper, two fibers are connected to the optical waveguides (gripping arms), one as input and the other as output. The fibers are also bonded to the outside of the hollow 
cylinder to provide mechanical robustness to the system. The shaft passes through the inside of the cylinder, having one end bonded to the backbone and the other end bonded to the external actuator mechanically fixed in space with the cylindrical support. The system requires an external actuator to be able to induce motion on the shaft which is connected to the backbone. Figure 3.3 presents a diagram of the MOEMS microgripper in which all its components are named using the terminology followed throughout this work.

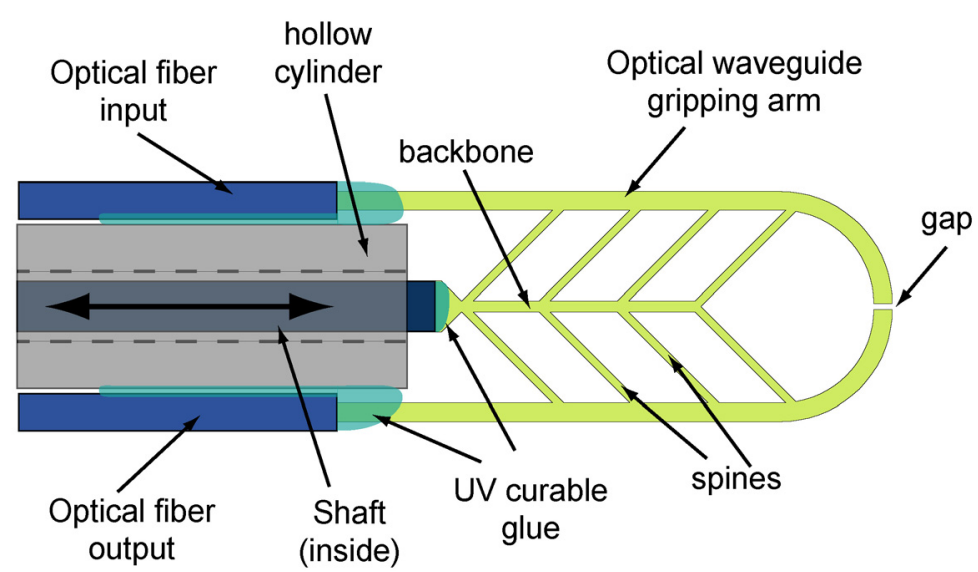

Figure 3.3. MOEMS microgripper showing its different components. Notice that the gripping elements are also optical waveguides so not only do they provide the mechanical means to manipulate microstructures, but also carry light to and from the microstructure to be manipulated.

The micrometer actuator approach requires the turning of a micrometer screw, which provides an accuracy of approximately $1 \mu \mathrm{m}$, however the vibrations introduced by turning the actuator could reduce the precision with which the microgrippers operate. In a case where more accuracy is required, piezo-electric actuation is more suitable, furthermore a higher degree of automation is enabled since it only requires an electric signal to operate. The system arrangement is the same, except that in this case the backbone displacement is induced by the expansion of the piezo-electric actuator. Another advantage of this second actuation approach is that the system can be 
implemented in a more compact manner due to the smaller dimension of the piezoactuator compared to the micrometer screw. It should be mentioned that the micrometer screw provides an easy method of actuation since it provides the possibility of pushing and pulling therefore allowing the microgripper to increase or decrease its initial gap. The piezo-electric actuator is limited to only pushing; this issue can be addressed by assembling the microgripper in a normally closed arrangement, placing the shaft under tension before fixing the actuator in place, in a way that when no voltage is applied, the microgripper is completely closed, and as voltage is applied, the microgripper starts to open.

\subsection{Fishbone analytical model}

Independently of the external actuator of choice, the microgripper opening and closing operation is controlled by the fishbone structure located between the gripping arms. Figure 3.4a portrays a microgripper in its relaxed state in which the design parameters are indicated as: initial gap $g_{0}$, initial spine angle $\theta_{0}$, and spine length $L$.

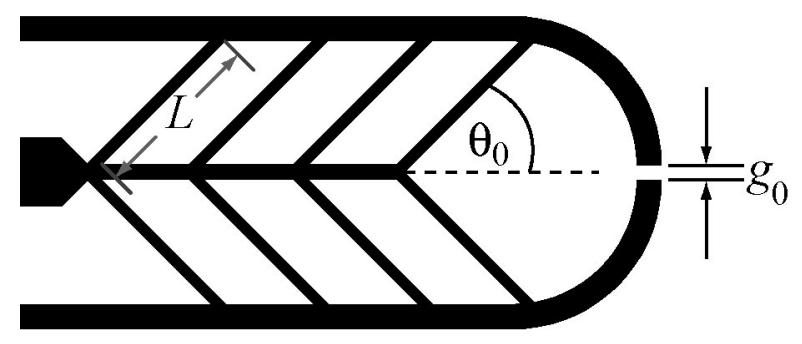

(a)

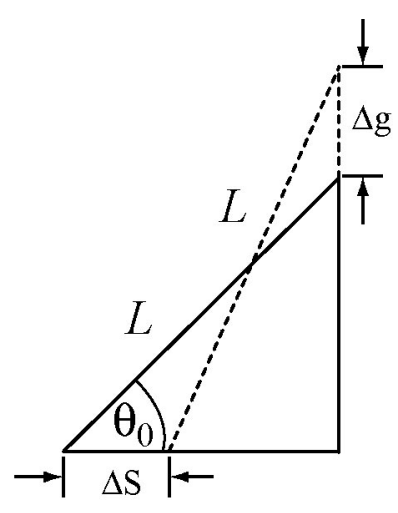

(b)

Figure 3.4. Analytical modeling of fishbone mechanism. a) microgripper with relaxed fishbone showing the initial gap $g_{0}$, initial spine angle $\theta_{0}$, and spine length $L$. b) Geometrical representation of gap variation $\Delta g$ by moving the backbone by $\Delta S$. 
Upon actuation, that is, when inducing a backbone displacement $\Delta S$, the gripping armspine joints move outwards by $\Delta g$ which consequently increases the initial gap by $2 \Delta g$, given that two arms contribute to this variation (figure 3.4b). For either of the two different actuation methods that cause a mechanical displacement of the backbone (micrometer screw or piezo electric), the dimension of the microgripper gap $(G)$ is therefore given by given by:

$$
G=g_{0}+2 \cdot \Delta g
$$

Where:

$\Delta g$ : variation in the microgripper opening gap per arm $g_{0}$ : initial gap separation

By simple geometry (figure $3.4 \mathrm{~b}$ ), it is possible to find an approximate expression for $\Delta g$ as a function of the length of a spine, and the initial angle it makes with the backbone, noticing that since ideally the spine length $L$ does not change, upon actuation, it still forms a right triangle, and therefore the following Pythagorean expression is obtained:

$$
\left(\Delta g+L \sin \left(\theta_{0}\right)\right)^{2}+\left(L \cos \left(\theta_{0}\right)-\Delta S\right)^{2}=L^{2}
$$

Where:

$\Delta s:$ displacement induced by the shaft on the backbone

$L$ : length of a spine

$\theta_{0}$ : initial angle between spines and backbone

From equation $3.2, \Delta g$ can be expressed as:

$$
\Delta g=\sqrt{L^{2}-\left(L \cos \left(\theta_{0}\right)-\Delta S\right)^{2}}-L \sin \left(\theta_{0}\right)
$$


or:

$$
\Delta g=\sqrt{L^{2}\left(1-\cos ^{2}\left(\theta_{0}\right)\right)+2 L \cos \left(\theta_{0}\right) \Delta S-\Delta S^{2}}-L \sin \left(\theta_{0}\right)
$$

which becomes:

$$
\Delta g=\sqrt{L^{2} \cdot \sin ^{2}\left(\theta_{0}\right)+2 \cdot L \cdot \cos \left(\theta_{0}\right) \cdot \Delta s-(\Delta S)^{2}}-L \cdot \sin \left(\theta_{0}\right)
$$

\subsection{Gap $(G)$ rate of change dependence on initial spine angle $\left(\theta_{0}\right)$}

One of the parameters that depends on the initial spine angle is the opening range of the microgripper. Figure 3.5 shows the relation between the gap and the displacement induced by the shaft (predicted for equation 3.5), for a fixed spine length $\mathrm{L}=354 \mu \mathrm{m}$ implemented in this work, at three different initial angles: $22.5^{\circ}, 45^{\circ}$ and $67.5^{\circ}$. It should be mentioned that maintaining the spine length constant requires adjusting the dimensions of the microgrippers arms bending radius to maintain a constant initial gap $g_{0}$. Theoretically, the maximum displacement, or maximum gap is achieved when the angle between the spine and the backbone becomes 90 degrees, which corresponds to a backbone displacement $\Delta s=L \cos \left(\theta_{0}\right)$. The curves presented in figure 3.5 are plotted up to the theoretical maximum displacement value, where a maximum theoretical gap is observed, and it can be observed that the rate of change of the gap approaches zero as $\Delta s$ approaches $L \cos \left(\theta_{0}\right)$. Furthermore, it is expected that increasing or decreasing the initial spine angle $\theta_{0}$ would directly translate into an increase or decrease of the gap $G$ rate of change with respect to the displacement induced on the backbone $(\Delta G / \Delta S)$, or in other words, the degree of precision on the gap variation upon actuation. Consequently, if a higher degree of control is required on the gap dimension, the microgripper could be fabricated with the spines at a higher than 45 degree angle, however this would reduce 
the opening range of the gripper. Furthermore, if a larger gap range is required, the microgripper could be fabricated at a lower than 45 degrees, but as expected, this would reduce the level of control over the gap. The microgrippers presented in this work were fabricated with the spines at $45^{\circ}$ as a good compromise for the range and control, with an initial gap $g_{0}$ of $16 \mu \mathrm{m}$.

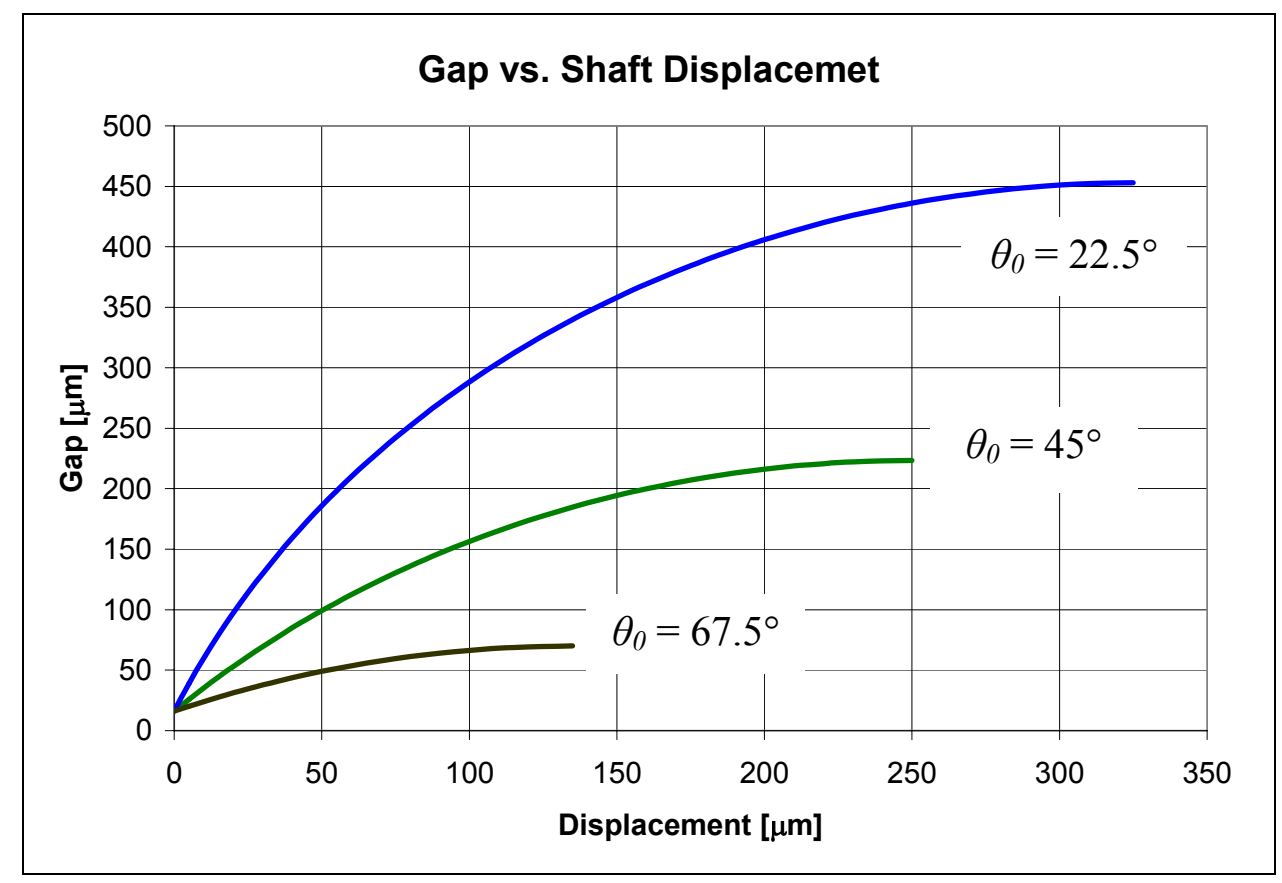

Figure 3.5. Theoretical gap range vs. shaft displacement dependence on initial spine angle $\theta_{0}$ predicted by equation 3.5. Curves are plotted up to the maximum theoretical gap value.

Another interesting variation of the previously discussed case is to study the case of implementing the microgrippers with spines at $22.5^{\circ}, 45^{\circ}$ and $67.5^{\circ}$, however, maintaining the distance between the gripping arms and the backbone constant, avoiding the need to adjust their bending radius; fact that would make it possible to assemble the microgrippers using the same hollow cylinder (refer to figure 3.3). As mentioned before, the spine length implemented in this work (at $45^{\circ}$ ) is $\mathrm{L}=354 \mu \mathrm{m}$. However, if the spine angle is to be modified, given that a constant gripping arm-backbone distance is to be 
maintained, the spine length should be adjusted accordingly. For instance, if the angle between the spines and the backbone is $22.5^{\circ}$ (half of $45^{\circ}$ ), the spines should be $654 \mu \mathrm{m}$ long, and if the angle would be $67.5^{\circ}$ (one and a half times $45^{\circ}$ ) the spines would be $271 \mu \mathrm{m}$ long. Figure 3.6 shows the relation between the gap and the displacement induced by the shaft for a fixed gripping arm-backbone separation, and spines at $22.5^{\circ}$, $45^{\circ}$, and $67.5^{\circ}$. Even though the gap does not vary linearly with the shaft displacement (equation 3.5), for small backbone displacements, the gap rate of change corresponding to these angles can be approximated to $\Delta G / \Delta S=4.5,2.0$, and 0.8 respectively. As mentioned before, it becomes evident that if a higher degree of control is required for more precision on the gap dimension, the microgripper should be fabricated with spines at an angle higher than 45 degrees, at the expense of smaller range.

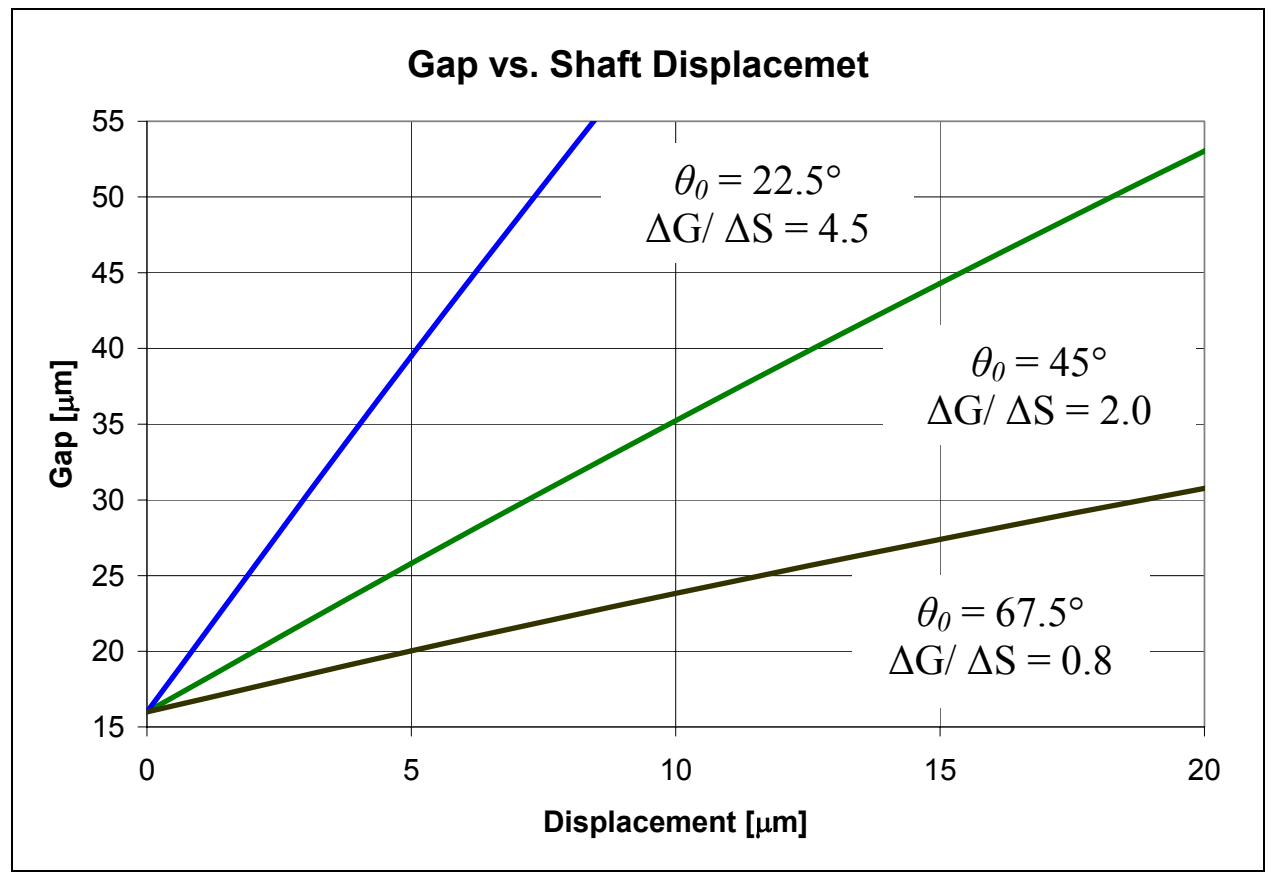

Figure 3.6. Relation between the gap and the displacement induced by the shaft for a fixed gripping armbackbone separation, and spines at $22.5^{\circ}, 45^{\circ}$, and $67.5^{\circ}$. Notice that higher angles provide smaller rates of change, and therefore more control over the gap dimensions. 


\subsection{Scaling laws}

The range of sizes of microstructures that the MOEMS microgrippers are able to grasp, the frequency at which they can operate, as well as the range of forces that the microgrippers apply when grasping a microobject, are parameters that depend on the microgrippers' overall dimensions. Furthermore, optical properties are also dependent on the physical dimensions of the microgrippers. In the following subsections mechanical and optical scaling laws are discussed.

\subsubsection{Mechanical scaling}

Scaling the microgrippers to smaller dimensions would allow the manipulation of smaller microobjects. This scaling would involve a change in the mechanical properties of the microgrippers such as spring constant and frequency response. The scaling law of the microgrippers spring constant can be illustrated with a simple analogous device, a cantilever with one end fixed, noticing that each of the gripping arms can be seen as cantilevers, with the load distributed among the points where the spines are joined to them. The spring constant of a cantilever flexed in the direction normal to its smallest dimension, or in other words its thickness, and parallel to its width, is given by:

$$
k=\frac{E w t^{3}}{4 l^{3}}
$$

where $E$ is the material Young's modulus, $w, t$, and $l$ are the width, thickness, and length of the cantilever respectively. If the microgrippers are scaled in an isomorphic manner, that is, all the dimensions are scaled by a factor $S$, and given that $E$ is ideally a 
dimensional-invariant material property, equation 3.6 could be written as equation 3.7 from where it can be seen that the spring constant is proportional to $S^{1}$.

$$
k=\frac{E S w(S t)^{3}}{4(S l)^{3}} \propto S^{1}
$$

For instance, if the microgrippers are scaled to $10 \%$ of their size $(S=0.1)$ then the spring constant will scale equally to $10 \%$ of its initial value.

The frequency response scaling law of the microgrippers can be determined analyzing the scaling of a simple mass spring system, for which the resonant frequency is given by:

$$
f_{0}=\frac{1}{2 \pi} \sqrt{\frac{k}{m}}
$$

where $k$ is the spring constant, and $m$ is the mass. From equation 3.7 it was determined that the spring constant scales as $S^{1}$, and it can be easily demonstrated that the mass scales as $S^{3}$. Consequently, equation 3.8 can be rewritten as equation 3.9 from where it can be seen that the natural resonant frequency changes as $S^{-1}$.

$$
f_{0}=\frac{1}{2 \pi} \sqrt{\frac{S k}{S^{3} m}} \propto S^{-1}
$$

For instance, if the microgrippers are scaled to $10 \%$ of their size $(S=0.1)$ then the resonant frequency will increase by a factor of 10 .

\subsubsection{Optical scaling laws}

Scaling the microgrippers isomorphically involves the reduction in size of the waveguide-gripping arms, which would cause the number of modes guided by the waveguides to be reduced. This reduction would continue up to the point of transition 
from a multimode waveguide to single mode. For instance, for SU-8, this transition would take place when the width of the waveguides reaches approximately $300 \mathrm{~nm}$ for $\lambda=635 \mathrm{~nm}$. If the microgripper is implemented in silicon, the transition to single mode takes place at similar dimensions for $\lambda$ in the infrared range, given that the index of refraction of silicon is higher than the index of SU-8, but the wavelength is longer.

Maintaining the waveguide widths, but scaling the spines width would have an effect in the optical losses introduced by the spines; in other words, the narrower the spines, the less losses introduced on the waveguides. Similarly, the width of the spines could be kept constant as the width of the waveguides is changed (present work); however in this case, the wider the waveguides, the less losses the spines introduce. Intuitively, the losses introduced by the spines increase significantly when the waveguide width to spine width ratio is less than unity. This statement will be verified through simulations in the following chapter. It should be kept in mind that even though having a large waveguide width to spine width ratio would be more efficient optically, it could compromise the mechanical integrity of the microgrippers, specifically the actuation mechanism. 


\section{SIMULATIONS}

\subsection{Mechanical simulations}

The MOEMS microgrippers mechanical performance was verified by simulations using COMSOL Multiphysics, a finite element analysis simulations suite. The models simulated took into consideration physical properties of SU-8 such as Young's modulus $E=4.02 \mathrm{GPa}$, Poisson coefficient $v=0.22$, and density $\rho=1190 \mathrm{~kg} / \mathrm{m}^{3}[2]$.

\subsubsection{Finite element meshes}

The finite element analysis brakes the structure to be analyzed in several smaller elements forming a mesh, and then calculates the interaction among them, consequently the higher the number of elements in a mesh, the more accurate the solution will be. The meshes generated for the finite element simulation corresponding to the $50 \mu \mathrm{m}$ and $100 \mu \mathrm{m}$ microgrippers are presented in figure 4.1. The meshes are composed of 13951 and 11720 elements respectively; no noticeable increment in accuracy was observed for finer meshes.
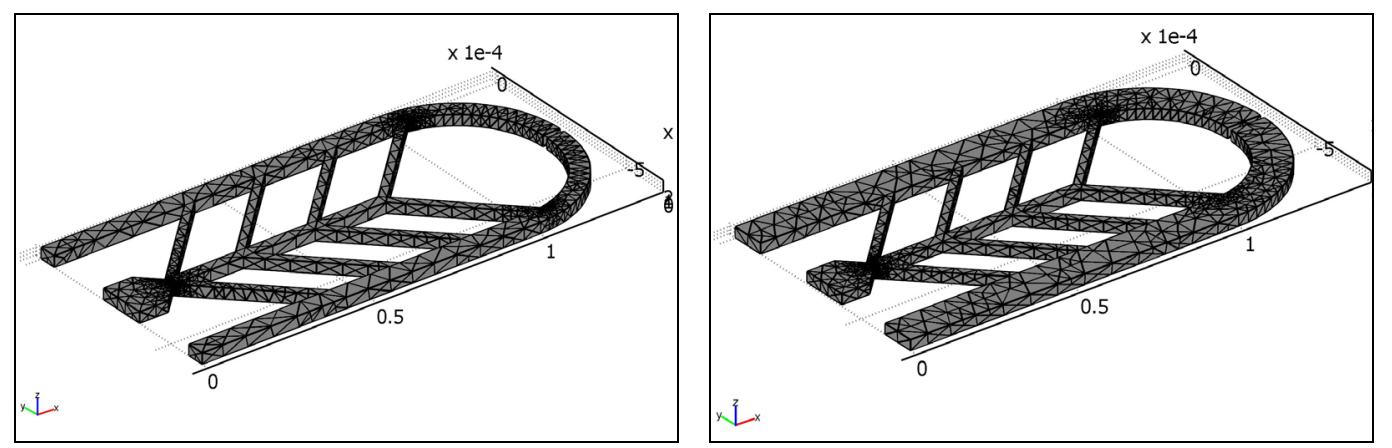

Figure 4.1. Meshes generated by COMSOL Multiphysics, used for finite element based simulation corresponding to the 50 (left) and $100 \mu \mathrm{m}$ (right) microgrippers. The meshes are composed of 13951 and 11720 elements respectively. 


\subsubsection{Fishbone actuator verification}

Simulations of the $50 \mu \mathrm{m}$ and $100 \mu \mathrm{m}$ wide arm microgrippers confirmed the functionality of the fishbone actuator. The behavior was predicted accurately for backbone displacements from $-8 \mu \mathrm{m}$ (pull backbone), at which both microgrippers closed, and for a displacement of $20 \mu \mathrm{m}$ (push backbone) at which both microgrippers opened from an initial gap of $16 \mu \mathrm{m}$ to a gap of $54 \mu \mathrm{m}$. These results are in agreement with the analytical model developed in section 3.5 , specifically with the graph presented in figure 3.6. Figure 4.2 shows the $50 \mu \mathrm{m}$ and $100 \mu \mathrm{m}$ microgrippers in their completely closed position after an $8 \mu \mathrm{m}$ backbone pull and opened to $\sim 54 \mu \mathrm{m}$ after a $20 \mu \mathrm{m}$ backbone push, where the levels of Von Mises stress post actuation are represented in a color scale, representing the lower limit (blue) minimum values while top limit (red) represents maximum levels. Von Mises stress is a parameter commonly used in finite element analysis simulation analysis which describes the total stress at a point, taking into consideration the principal stresses in three dimensions. It can be seen that when the microgripper is actuated, the locations of maximum stress are the "V" junctions that the spines form with gripping arms as well as with the backbone. From these simulations it was observed that the gripping facets remain parallel at all times within 1 degree, which is attributed to the fishbone actuator which imposes a parallel displacement on the gripping arms.

\subsubsection{Out of plane backbone actuation}

Even though the structure could be simulated in 2D, it was necessary to perform 3D simulations to analyze the effect of a non-planar actuation. This was studied by 

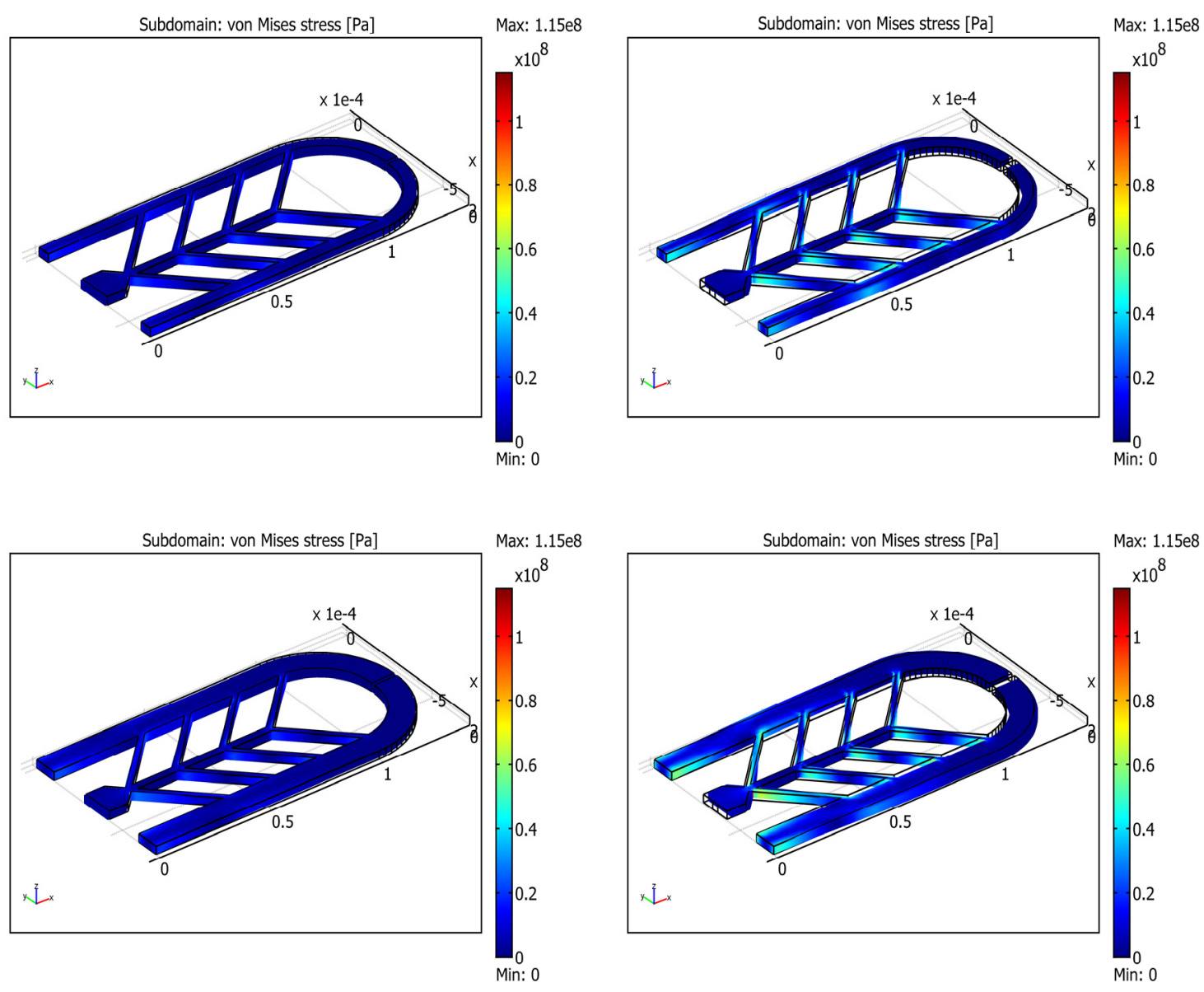

Figure 4.2. 3D Simulated microgrippers actuation (birds eye view) for an $8 \mu \mathrm{m}$ backbone pull closing the grippers and opened to $\sim 54 \mu \mathrm{m}$ after a $20 \mu \mathrm{m}$ backbone push. On the left: $50 \mu \mathrm{m}$ (top) and $100 \mu \mathrm{m}$ (bottom) gripper closed. On the right: $50 \mu \mathrm{m}$ (top) and $100 \mu \mathrm{m}$ (bottom) gripper opened. Color scale represents levels of Von Mises stress post actuation. The lower limit of the scale (blue) represents minimum levels while top (red) represents maximum levels.

adding a component to the displacement vector perpendicular to the actuation plane, applied to the backbone. It was observed that the effect of slight out-of-plane actuation is not critical. This is due to the extra spines attaching the backbone to the gripper arms which restrain the out-of-plane motion, and also because the backbone is displaced a very small percentage of the microgripper total length. This is an important observation since once the actual device is assembled, the actuation might not remain perfectly in plane. 


\subsubsection{Gripping force estimation}

When handling delicate samples such as cells, excessive gripping forces applied by the microgrippers could be damaging, and therefore it is important to determine the range of forces that the MOEMS microgrippers can apply to a microobject being held. The gripping force range was derived from mechanical simulations. When the backbone is pulled beyond the point of closing the microgripper $(\sim 8 \mu \mathrm{m})$, the reaction forces at the facets show a spring like behavior with effective spring constant of $0.118 \mathrm{mN} / \mu \mathrm{m}$ for a $50 \mu \mathrm{m}$ wide arm microgripper. Figure $4.3 \mathrm{~b}$ shows the force vs. additional backbone displacement relation, from where the spring constant was obtained. Fig 4.3a shows the simulation model used to extract this data, in which the backbone is pulled $30 \mu \mathrm{m}$ beyond closure.

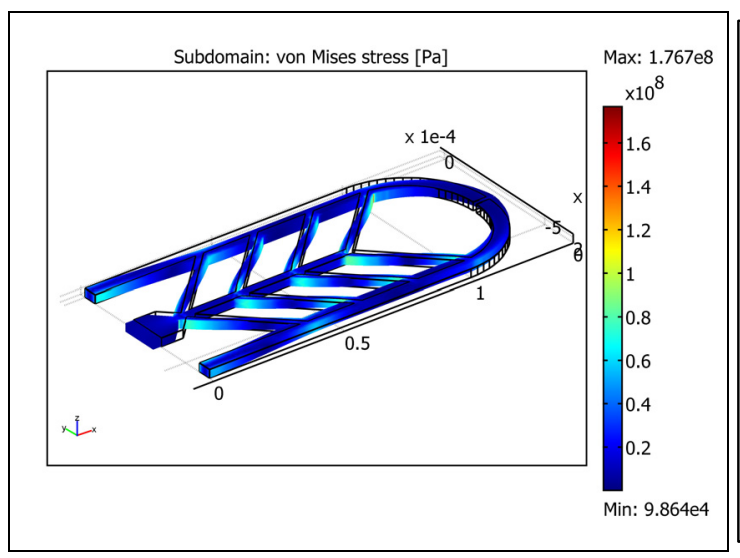

(a)

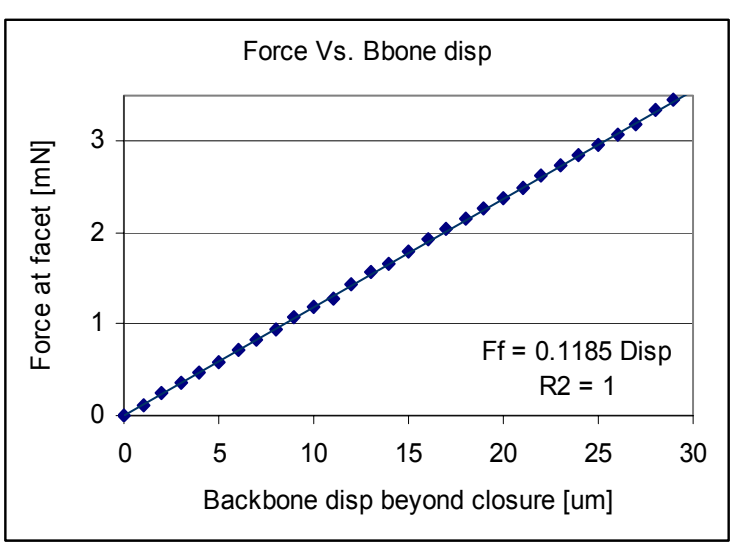

(b)

Figure 4.3. $50 \mu \mathrm{m}$ arm microgripper effective spring constant determination through simulations. Pulling the backbone beyond the microgripper closing point produces reaction forces between the facets, by which spring-like behavior is observed. a) backbone pulled $30 \mu \mathrm{m}$ beyond closure, b) Reaction forces at the facet vs. backbone additional displacement.

The effective spring constant of the microgripper depends on the mechanical properties of SU-8, as well as its geometry. One parameter in the geometry that allows tuning of the spring constant is the initial spine angle, as it affects the relation between 
the displacement of the backbone and the gap variation (figure 3.6). Consequently, the forces applied by the microgripper for a given backbone displacement, can be increased or reduced by modifying the spine angle. For instance, implementing the microgripper with spines at an angle grater than $45^{\circ}$ reduces the rate at which the gap varies for backbone displacements, consequently producing a lower spring constant. This would allow more control of the forces applied by the gripper, facilitating the manipulation of delicate samples such as cells. Furthermore, if the deformation induced on a cell by the gripper could be monitored (i.e. visually), it would be possible to determine its elastomechanical properties which provide information about the presence and progression of possible diseases [28]. For $0.01 \mu \mathrm{m}$ of additional backbone displacement beyond the closed point (corresponding to 0.1 volts increment in the piezo), the gripper applies a force of $1.18 \mu \mathrm{N}$; considering that for the $50 \mu \mathrm{m}$ arm gripper, the facets have an area of $50 \mu \mathrm{m} \times 30 \mu \mathrm{m}=1500 \mu \mathrm{m}^{2}$, the pressure applied is $787 \mathrm{~Pa}$, which is comparable to elastic parameters reported in [11] for cells such as fibroblasts, which is an indicator that the MOEMS microgrippers are suited for biological investigations on single cells.

\subsection{Optical waveguides/Gripping arms simulation}

Optical simulations were performed from which two kinds of losses were numerically estimated through simulations. The losses introduced by the gripping arm bends were simulated by the 3D-to-2D effective index method (finite difference time domain FDTD) and the losses introduced by the spines were estimated through three-dimensional Beam Propagation Method (3D-BPM), both by RSOFT, a photonics simulation suite. For these simulations the SU-8 index of refraction reported in the literature of $n=1.6$ was used, 
with an air cladding $n=1$, since the microgrippers are suspended in air. The resolution of the simulations grid was $100 \mathrm{~nm}$ in all three dimensions. The input light was distributed among the first 10 modes (fundamental plus modes 1 through 9) of each waveguide as it was determined also through simulations that most of the optical power is concentrated in these lower modes. Given that the cladding is air and it is uniformly distributed along the waveguide, the effective indexes for TE or TM polarization are quite similar, making light propagation almost polarization independent. The simulations presented here considered TE polarization.

\subsubsection{Losses due to spines}

The losses due to spines are present in both, the input and output waveguide, since both are connected to four spines. The worst case is observed at the output waveguide, where the spines make an acute angle with respect to the direction of the propagation of light. In order to study the effect of waveguide width, simulations were performed for the output waveguide with widths of $10,20,30,40,50$, and $100 \mu \mathrm{m}$, at a $30 \mu \mathrm{m}$ thickness. In all cases, the waveguide was simulated with four $20 \mu \mathrm{m}$ wide by $30 \mu \mathrm{m}$ thick spines at $45^{\circ}$. The light source was set to $532 \mathrm{~nm}$. Table 4.1 presents a summary of the losses observed for the six different cases; notice that the only case that shows a significant amount of losses is the $10 \mu \mathrm{m}$ waveguide, having $2.42 \mathrm{~dB}$ of losses. This was expected given that the spines are twice as wide as the waveguide, which corresponds to a waveguide width to spine width ratio less than unity, as discussed in Chapter III. However, it is also important to notice that already in the case of the waveguide having the same width as the spines ( $20 \mu \mathrm{m}$ gripper), the losses introduced by the four spines are 
only $0.24 \mathrm{~dB}$. Figure 4.4 presents the transmission losses along the waveguides (not including the bend); the curve corresponding to the $10 \mu \mathrm{m}$ microgripper goes off scale rapidly due to its relatively high losses when compared to the rest.

Table 4.1. Summary of total losses introduces by four $20 \mu \mathrm{m}$ spines at $45^{\circ}$ at the input waveguide.

\begin{tabular}{cc}
\hline $\begin{array}{c}\text { Width } \\
{[\mathrm{um}]}\end{array}$ & $\begin{array}{c}\text { Total losses due to } \\
\text { 20 um spines }[\mathrm{dB}]\end{array}$ \\
\hline 10 & 2.416 \\
20 & 0.242 \\
30 & 0.118 \\
40 & 0.042 \\
50 & 0.026 \\
100 & 0.002 \\
\hline
\end{tabular}

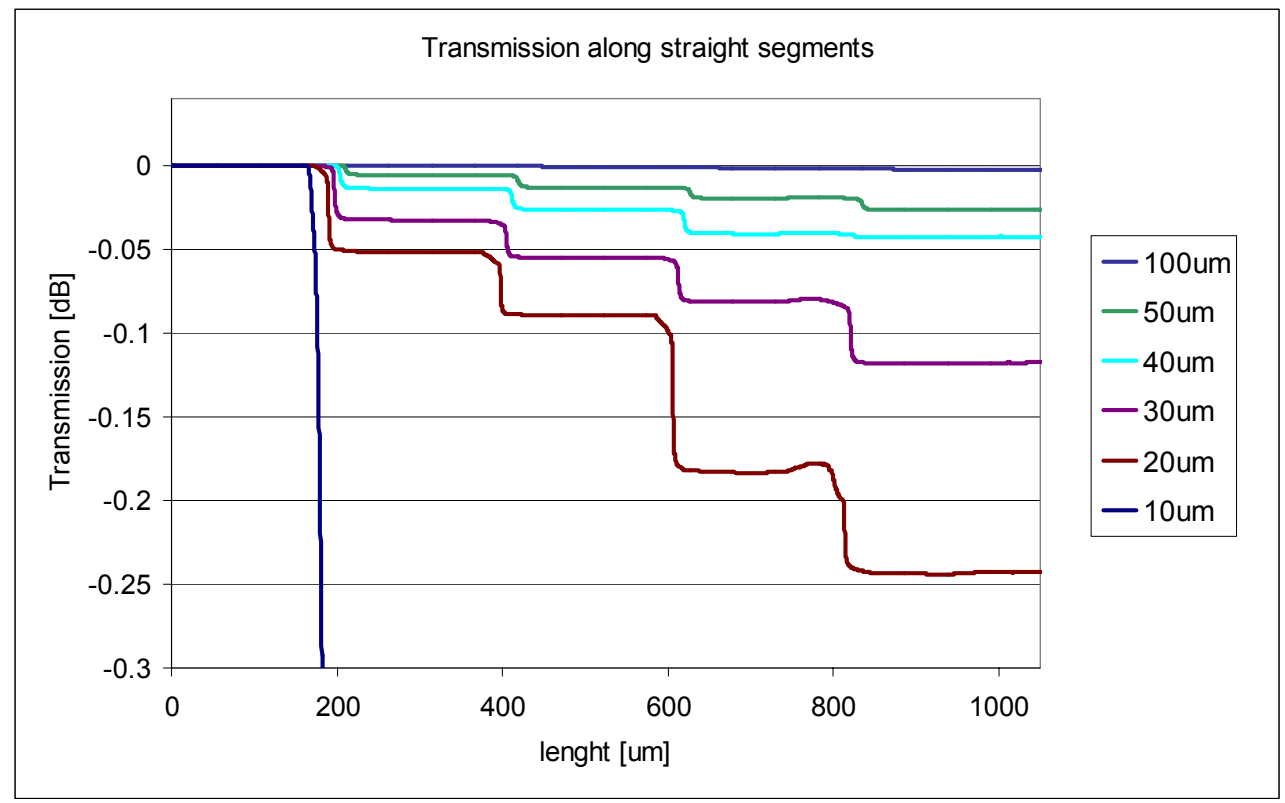

Figure 4.4. Staircase like transmission due to the four $20 \mu \mathrm{m}$ spines attached to the output waveguide, for microgrippers waveguide widths of $10,20,30,40,50$, and $100 \mu \mathrm{m}$. Notice that the losses introduced on the $10 \mu \mathrm{m}$ waveguide are significant, and therefore it goes rapidly out of the graph scale. 


\subsubsection{Bending losses}

Waveguide bends for the $10,20,30,40,50$, and $100 \mu \mathrm{m}$ wide waveguides were simulated. The bending radius for all the cases was $270 \mu \mathrm{m}$, measured from the center of the bend up to the center of the waveguide. Bending losses determined from these simulations are summarized in table 4.2. It should be mentioned that these simulations only predict the losses caused by the change of direction of the waveguide, and do not consider the losses that are present in the waveguide due to side wall roughness. In a later chapter, it will become evident that losses due sidewall roughness are noticeable; the source of this issue will be explained, and possible approaches to mitigate this roughness will be suggested. It should me mentioned that bending losses need to be investigated, given that a small bending radius is required to implement the gripping elements. Notice that larger bending radii would require longer spines, which could compromise the sturdiness of the structure, which would have an effect in its mechanical performance. The simulation results are presented graphically in figures 4.5 and 4.6, which show $532 \mathrm{~nm}$ light propagating along the waveguide bends of the $10,20,30,40,50$ and $100 \mu \mathrm{m}$ waveguides bent at a radius of $270 \mu \mathrm{m}$.

Table 4.2. Summary of bending losses for different waveguide widths at a radius of $270 \mu \mathrm{m}$

\begin{tabular}{cc}
\hline $\begin{array}{r}\text { Waveguide } \\
\text { width [um] }\end{array}$ & Bending losses [dB] \\
\hline 10 & 0.09 \\
20 & 0.11 \\
30 & 0.09 \\
40 & 0.12 \\
50 & 0.13 \\
100 & 0.13 \\
\hline
\end{tabular}




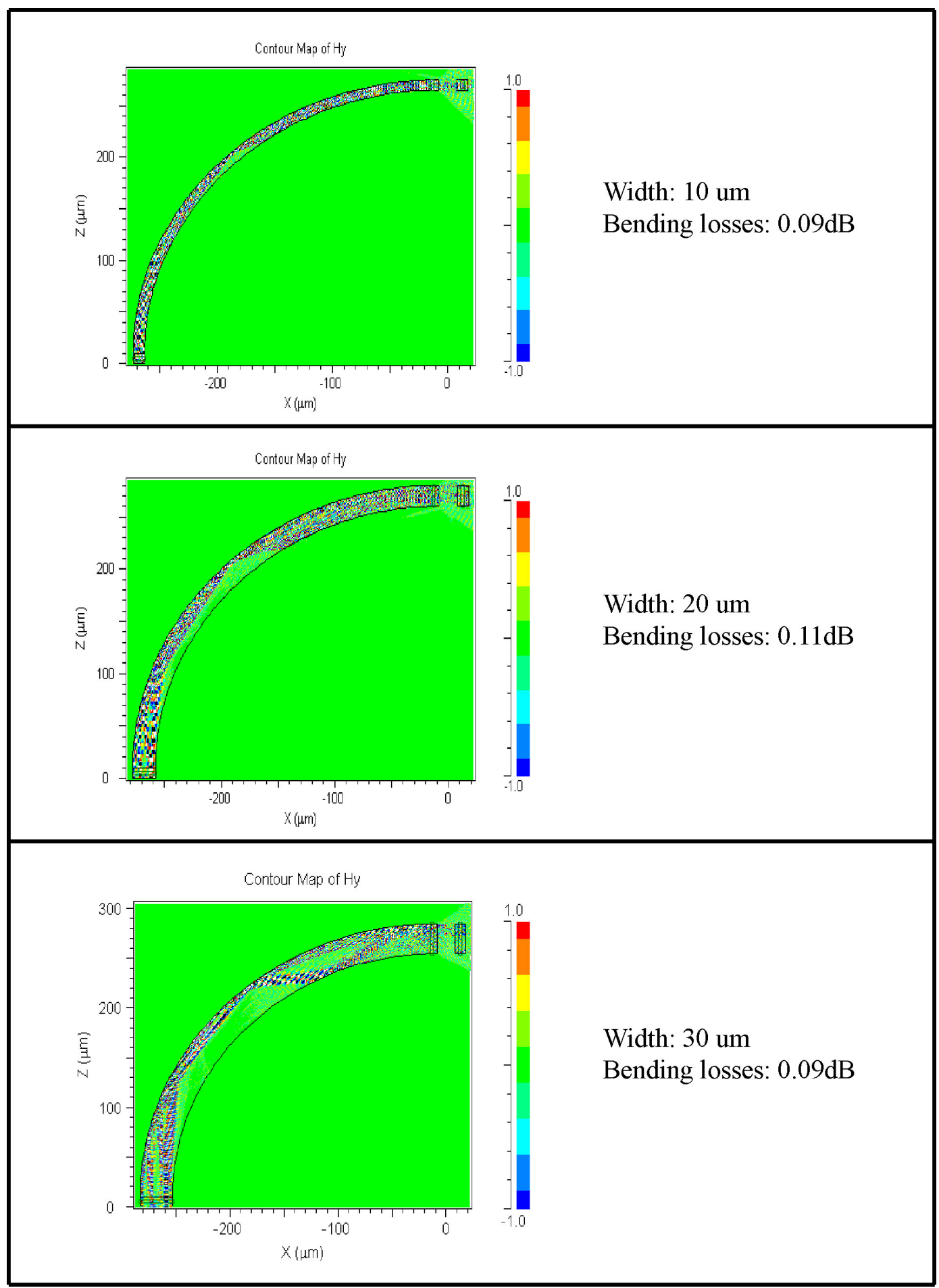

Figure 4.5. Light propagating along the waveguide bends corresponding to the 10,20 , and $30 \mu \mathrm{m}$ waveguides bent at a radius of $270 \mu \mathrm{m}$. The input light was distributed among the first 10 modes (fundamental plus modes 1 through 9) of each waveguide with TE polarization. 


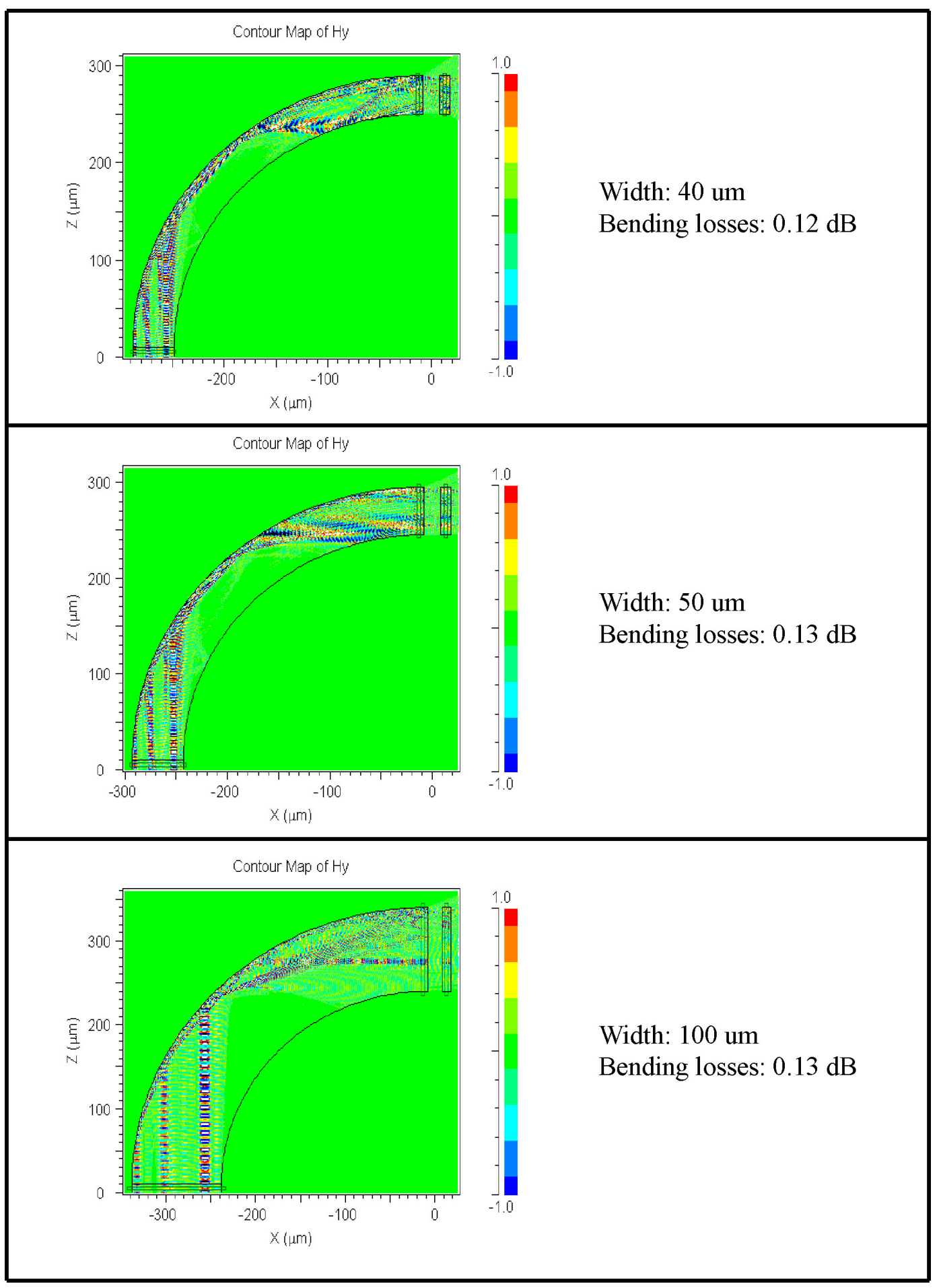

Figure 4.6. Continuation from previous figure. Light propagating along the waveguide bends corresponding to the 40,50 , and $100 \mu \mathrm{m}$ waveguides bends. 


\section{SYSTEM IMPLEMENTATION}

\subsection{Microfabrication}

The device microfabrication process was completely developed at Florida International University's Motorola Nanofabrication Research Facility (MNRF), a class 100/10000 cleanroom environment (http://www.eng.fiu.edu/ameri/). The microfabrication steps are explained in detail in the following subsections.

\subsubsection{Mask layout design}

The structure discussed in previous chapters, composed of flexible waveguides and an actuation mechanism (fishbone), can be physically implemented through different fabrication approaches. Typically, to produce a suspended structure, a sacrificial layer is patterned on a substrate, next the structural material is patterned, and then the sacrificial later is removed, requiring at least two photomasks. If the microgrippers would be implemented through this approach, they would be attached to a substrate, which would have to be cut to leave the microgripper arms protruding from the substrate edge [5], and finally the microgrippers waveguides would have to be bonded to optical fibers. Also, if several microgrippers are fabricated in a common substrate, more dicing would be required. The fabrication process flow followed in this work requires only one photomask, it allows the fabrication of several microgrippers in a common substrate, and does not involve dicing.

The mask layout is comprised of several relatively large frames that support the microgrippers. The pattern in the mask is prepared to allow a fast complete release of the frames supporting the microgrippers, and for this purpose the frames contain an array of 
$50 \mu \mathrm{m}$ by $50 \mu \mathrm{m}$ orifices with a pitch of $150 \mu \mathrm{m}$ leading to approximately $10 \%$ hole coverage, to allow a uniform sacrificial layer removal. Each frame includes microgrippers with gripping arms widths of $10,20,30,40,50$, and $100 \mu \mathrm{m}$, two of each. Each microgripper is attached to the a main frame by means of thin stubs, which are strong enough to maintain each microgripper in place during the microfabrication process, but weak enough to break once the microgripper has been bonded to the actuation part of the system. It is worth mentioning that a non-uniform etch of the sacrificial layer would lead to microgrippers being released much before the main frames, which would increases the chances of the stubs breaking prematurely. The mask includes two sets of frames, one that supports microgrippers designed for mechanical-only operation and characterization purposes, and a second that supports microgrippers in their final design form. The main difference between these two kinds of frames is that the first one includes a set of microgrippers with short gripping arms (flush with the backbone),

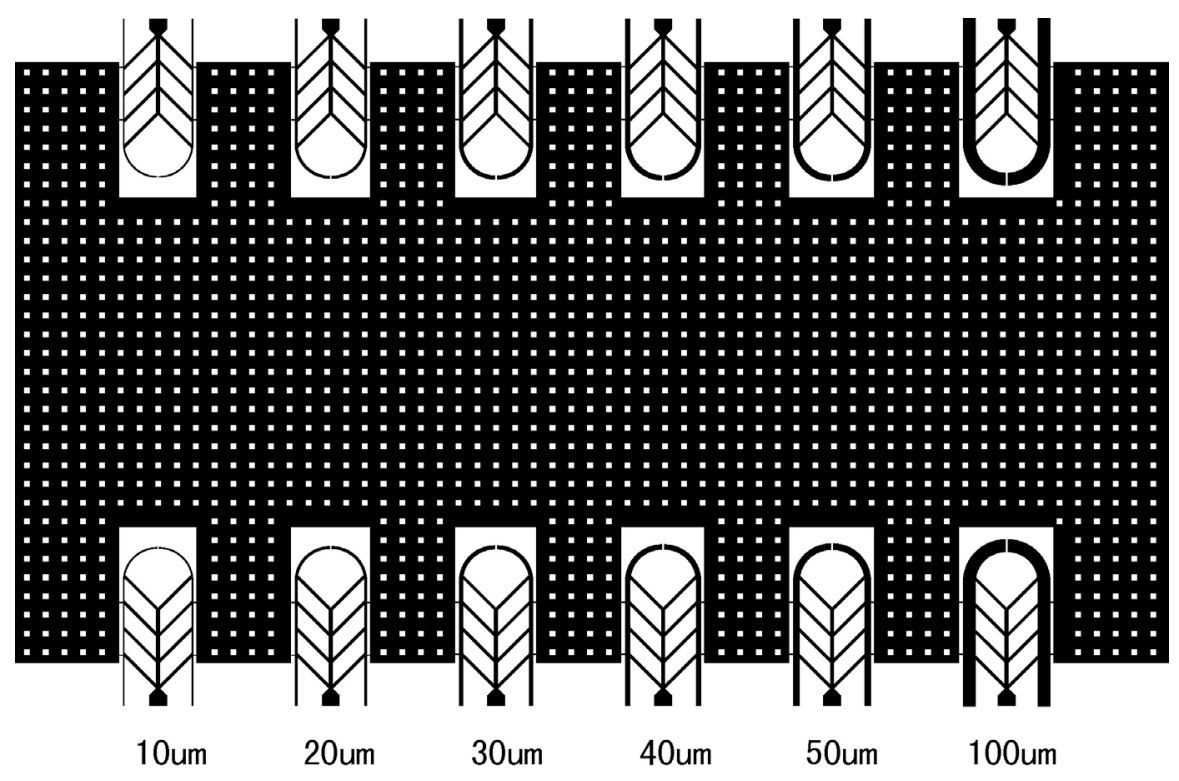

Figure 5.1. Layout of frame supporting 12 microgrippers of 10, 20, 30, 40, 50, and $100 \mu \mathrm{m}$ gripping arm width, two of each, designed to be utilized for mechanical testing only. Notice that the microgripper arms are flush with the backbone. 


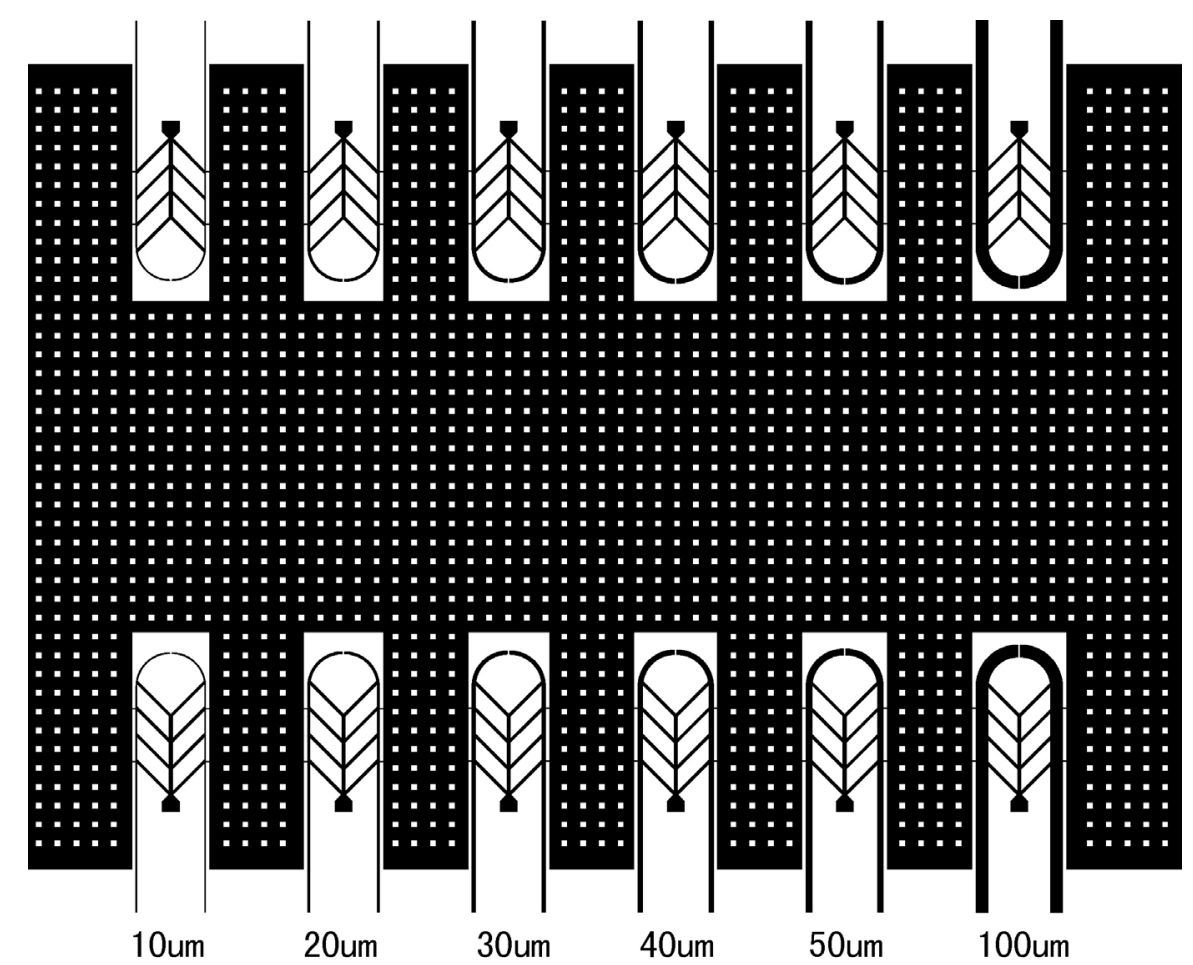

Figure 5.2. Layout of frame supporting microgrippers in their final design form, with elongated gripping arms to be bonded to optical fibers. The microgrippers are attached to a large frame by means of thin stubs strong enough to hold each microgripper in place during microfabrication, but weak enough to break once the microgripper has been bonded to the hollow cylinder, actuator and optical fibers.

and the second kind includes grippers with elongated gripping arms, meant to be bonded to optical fibers; this will become evident later in section 5.2, where the system assembly process is explained in detail. The two types of frames are presented in figures 5.1 and 5.2 respectively. The CAD layout was prepared using LayoutEditor ${ }^{\mathrm{TM}}$ (freeware available online at http://layout.sourceforge.net/), and the photomask was fabricated in house using the uPG101 table top maskless lithography system from Heidelberg.

\subsubsection{Substrate preparation}

The fabrication process is starts with a silicon substrate with $2 \mu \mathrm{m}$ of silicon dioxide, used as a complete sacrificial layer (figure 5.3, step 1). The substrate is first spin-cleaned with acetone followed by isopropanol. In order to promote adhesion 
between SU-8 and the oxide, the substrate is treated for 60 seconds in a reactive ion etching (RIE) oxygen plasma, using 100 Watts of RF power, a pressure of 175 mTorr, and $\mathrm{O}_{2}$ gas flow of $10 \mathrm{sccm}$ (standard cubic centimeter per minute).

\subsubsection{Photolithography}

The substrate is spin-coated at 2000 RPM with SU-8 25 to a thickness of $30 \mu \mathrm{m}$ (figure 5.3, step 2), pre-baked for $13 \mathrm{~min}$ at $95^{\circ} \mathrm{C}$ and exposed with I line illumination in a contact aligner (figure 5.3, step 3) given that wavelengths of 350 to $400 \mathrm{~nm}$ are needed to expose SU-8, followed by a hotplate post-exposure-bake (PEB) at $95^{\circ} \mathrm{C}$ for 16 minutes and developed using PGMEA (propylene glycol monomethyl ether acetate) (Figure 5.3, step 4). Initial experiments were carried out to test direct write with the uPG101, however the $30 \mu \mathrm{m}$ SU-8 film was not sufficiently sensitized at the maximum dose available in the machine; since then the machine's laser was changed increasing the dose available, however it is only enough to expose $\mathrm{SU}-8$ films less than $1 \mu \mathrm{m}$ thick. The specs corresponding to SU-8 25 recommend an exposure dose between 150 to $300 \mathrm{~mJ} / \mathrm{cm}^{2}$ [14]; however, in order to optimize the lithography conditions, an exposure matrix was executed, to obtain the required dose to achieve the needed resolution, and to produce vertical walls which are specially important at the gripping facets. Non-verticalities could impair the ability of the microgripper to grasp microobjects, and would also have an effect on the light coupling between the gripping facets. The mask aligner utilized is equipped with a $12.5 \mathrm{~mW} / \mathrm{cm}^{2}$, hence to obtain doses ranging from 100 up to $300 \mathrm{~mJ} / \mathrm{cm}^{2}$ with increments of $50 \mathrm{~mJ} / \mathrm{cm}^{2}$; the exposure time was varied from 8 to 24 seconds in increments of 4 seconds. SEM images of the obtained results are presented in figure 5.4., 
monitoring the facets of the $10 \mu \mathrm{m}$ wide arm microgripper, given that they require the highest resolution. From these results it was decided that the adequate dose corresponded to 24 seconds, which corresponds to $300 \mathrm{~mJ} / \mathrm{cm}^{2}$. Since this result corresponds to the upper limit of the matrix, higher doses were tested, however, no significant improvement was observed, on the contrary, symptoms of over exposure were detected at these higher doses as the wall profile started to turn positive. It is worth mentioning that the wall profile can be also improved by filtering out excessive energy from the lamp below $350 \mathrm{~nm}[14]$.

1

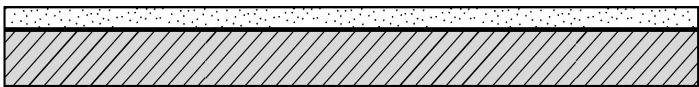

2
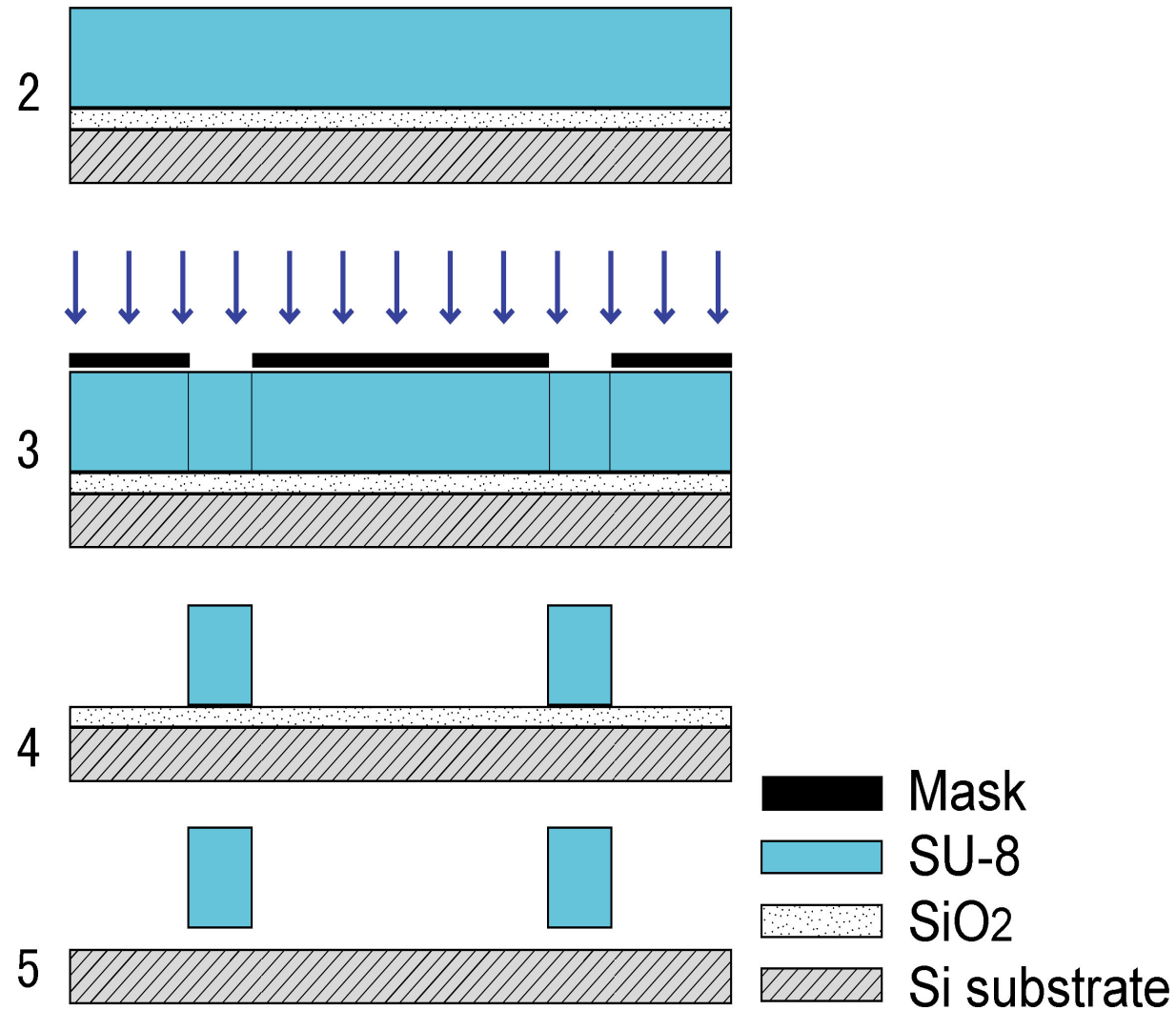

Figure 5.3. Fabrication process steps: 1: Silicon substrate with $2 \mu \mathrm{m}$ of $\mathrm{SiO}_{2}$, 2: SU-8 Spin-coat, 3: UV exposure, 4: Develop, 5: Complete structure release in BOE. 


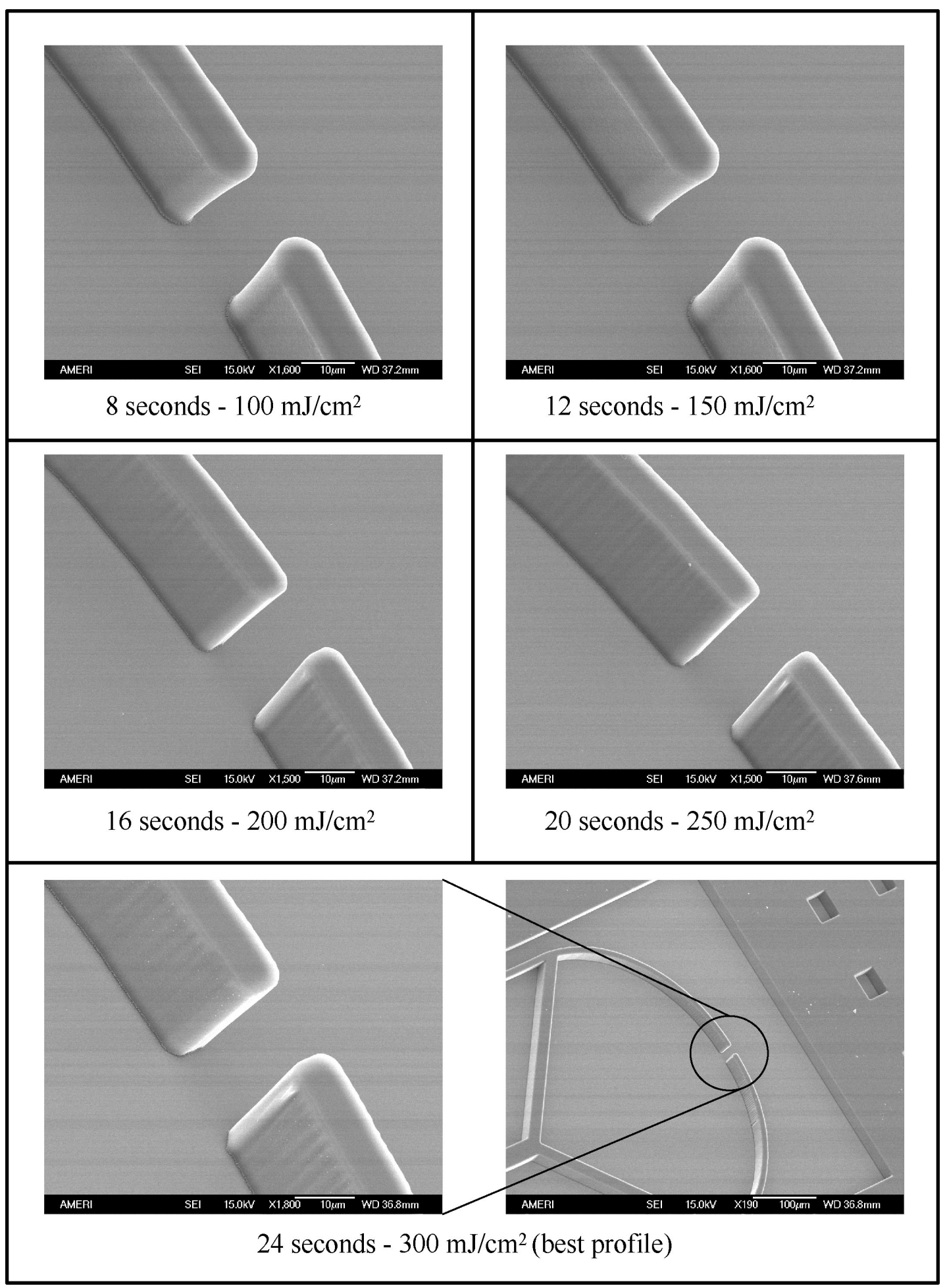

Figure 5.4. Exposure matrix results for SU-8 at $30 \mu \mathrm{m}$ thickness, monitoring the profile of the $10 \mu \mathrm{m}$ arm microgripper, since it has the smallest critical dimension. 8 - 24 seconds exposures corresponding to 100 $300 \mathrm{~mJ} / \mathrm{cm}^{2}$ doses. The best profile was observed with the 24 second dose. 


\subsubsection{Release}

Subsequent to the SU-8 layer patterning and inspection, the complete structure is released by immersing the substrate in buffered hydrofluoric acid (buffered oxide etch, BOE) for approximately 10 minutes (figure 5.3, step 5). The previously described perforated mask enables a uniform silicon dioxide release etch. Once the structure is released, the SU-8 devices come afloat on the acid surface, making it possible to pick them up manually. Each structure is then rinsed in deionized (DI) water and placed in an absorbent tissue; such drying technique is required in this case since blow drying would be destructive. It should be mentioned that the SU-8 microgrippers do not float in either water or isopropanol, however, they do float on BOE. Figure 5.5 presents SEM images of a released frame, showing a close-up of the microgripper with $30 \mu \mathrm{m}$ wide gripping arms. At this point the microgrippers are ready for assembly.

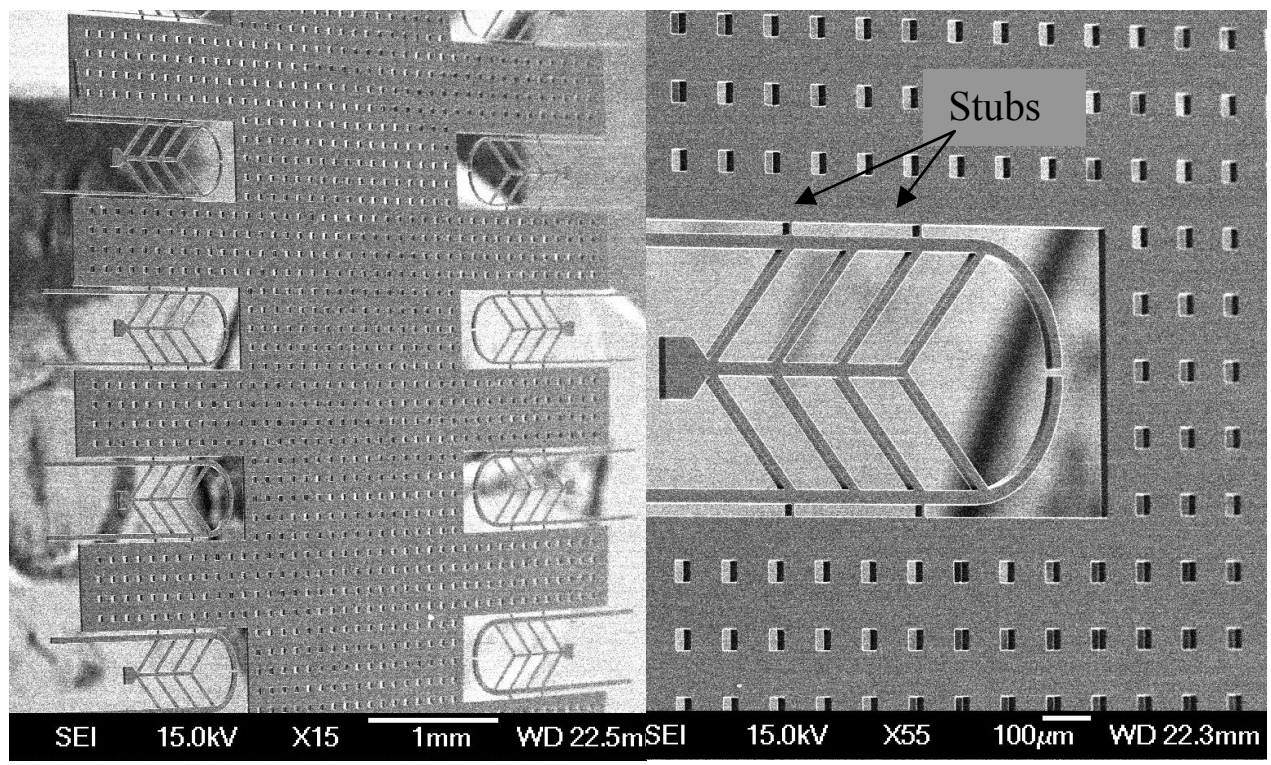

(a)

(b)

Figure 5.5. (a) SEM micrograph of released main frame supporting MOEMS microgrippers, (b) Close-up detail of $30 \mu \mathrm{m}$ arm width microgripper supported by 4 stubs. 


\subsection{Assembly}

The first attempts of assembling the system involved only connecting the released microgrippers to the external actuator (micrometer screw or piezoelectric actuator), with the goal of experimentally verifying the mechanical functionality of the grippers, and evaluating the fishbone actuation mechanism performance [29]. For this, one main frame containing one set of microgrippers is placed on an XYZ stage. Then a $100 \mu \mathrm{m}$ shaft (already attached to the actuator of choice), is visually aligned to the backbone of a microgripper of the desired size under a stereoscopic microscope, with its tip previously coated with UV curable glue. Once the desired alignment is achieved, the backbone is brought in contact with the shaft and UV cured, producing a strong bond. In a similar manner, the microgripper arms are bonded to the end of the hollow cylinder. At this point the microgripper is ready to be detached from the main frame. A slight pull on the mainframe is enough to break the supporting stubs, completing the assembly process. The result is a microgripper attached to a support large enough to be easily manipulated. Figure 5.6 presents a $100 \mu \mathrm{m}$ wide arm microgripper assembled, along with a diagram

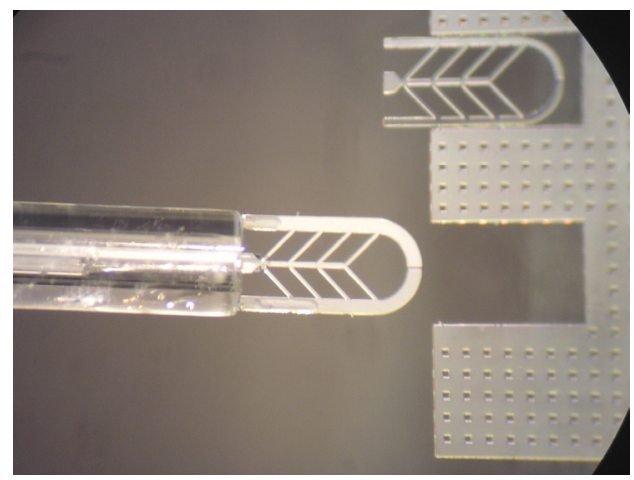

(a)

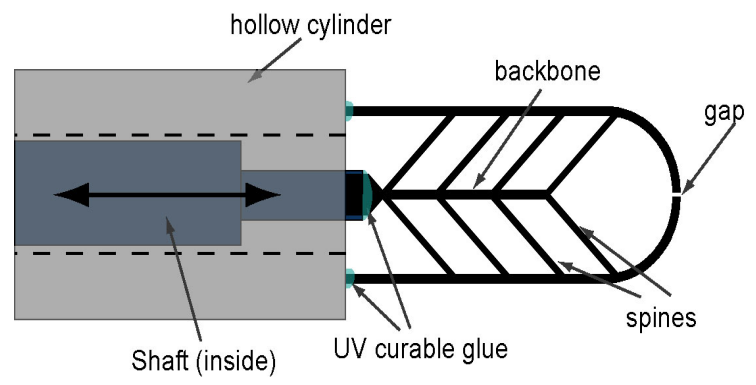

(b)

Figure 5.6. a) Completion of microgripper assembly for mechanical operation-characterization only. Notice that there are no optical fibers present. b) Microgripper diagram showing its different components. 


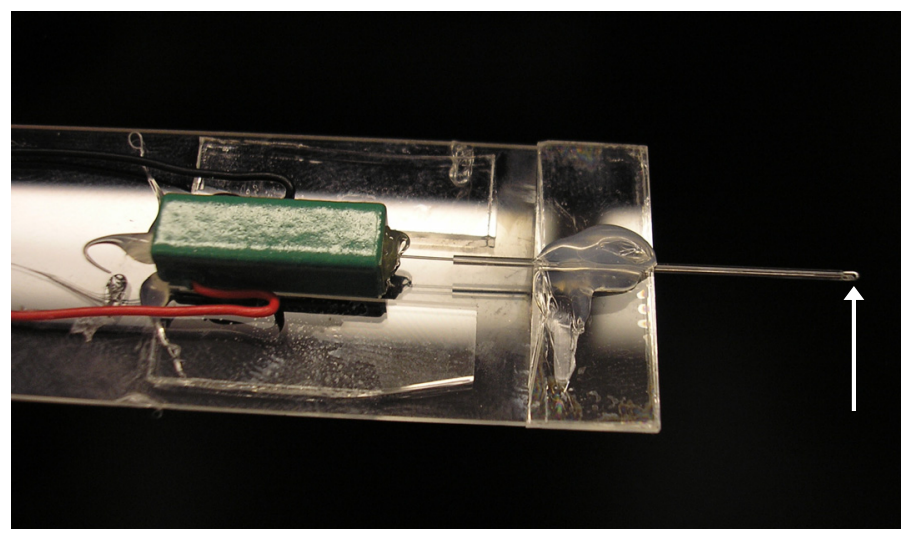

Figure 5.7. Piezoelectric actuator/microgripper assembled system. The arrow points to the microgripper indicating the different components. Figure 5.7 shows the assembled system for mechanical operation-characterization only (using a piezoelectric actuator), mounted onto a microscopy glass slide for ease of use in a high resolution optical microscope.

After the mechanical functionality has been verified experimentally, a more elaborated assembly process was carried out to include the optical fibers in the system, and therefore be able to test the main property of the MOEMS microgrippers. It should be mentioned that the length of the waveguide arms needs to be longer to allow simultaneous bonding to the optical fibers and to the hollow cylinder side walls [30]. Microgrippers with elongated legs presented in figure 5.2 are utilized.

To perform the actuator-microgripper-optical fibers assembly, the main frame is placed on an XYZ stage. The shaft (already attached to the actuator), with its tip previously coated with UV glue, is visually aligned to the backbone of a microgripper of the desired size under a stereoscopic microscope. Once the desired alignment is achieved, the backbone is brought in contact with the shaft and UV cured, producing a mechanically strong bond, as shown in figure 5.8-1. At this point, an optical fiber, secured on a second XYZ stage, is carefully aligned to the waveguide input (tip is coated 
with UV curable glue). The alignment process is carried out with the source laser on $(10 \mathrm{~mW}, 632.8 \mathrm{~nm}$ laser used only for assembly), in a way that, when maximum light coupling is determined, the glue is cured to maintain the aligned configuration (see figure 5.8-2). The second fiber is aligned and attached to the microgripper using the same technique. Some extra glue is added between the fibers and the supporting hollow cylinder to provide more robustness to the system. At this point the microgripper is ready to be detached from the main frame. A slight pull on the mainframe is enough to break the supporting stubs, completing the assembly process. The finished device is presented in Figure 5.8-4.

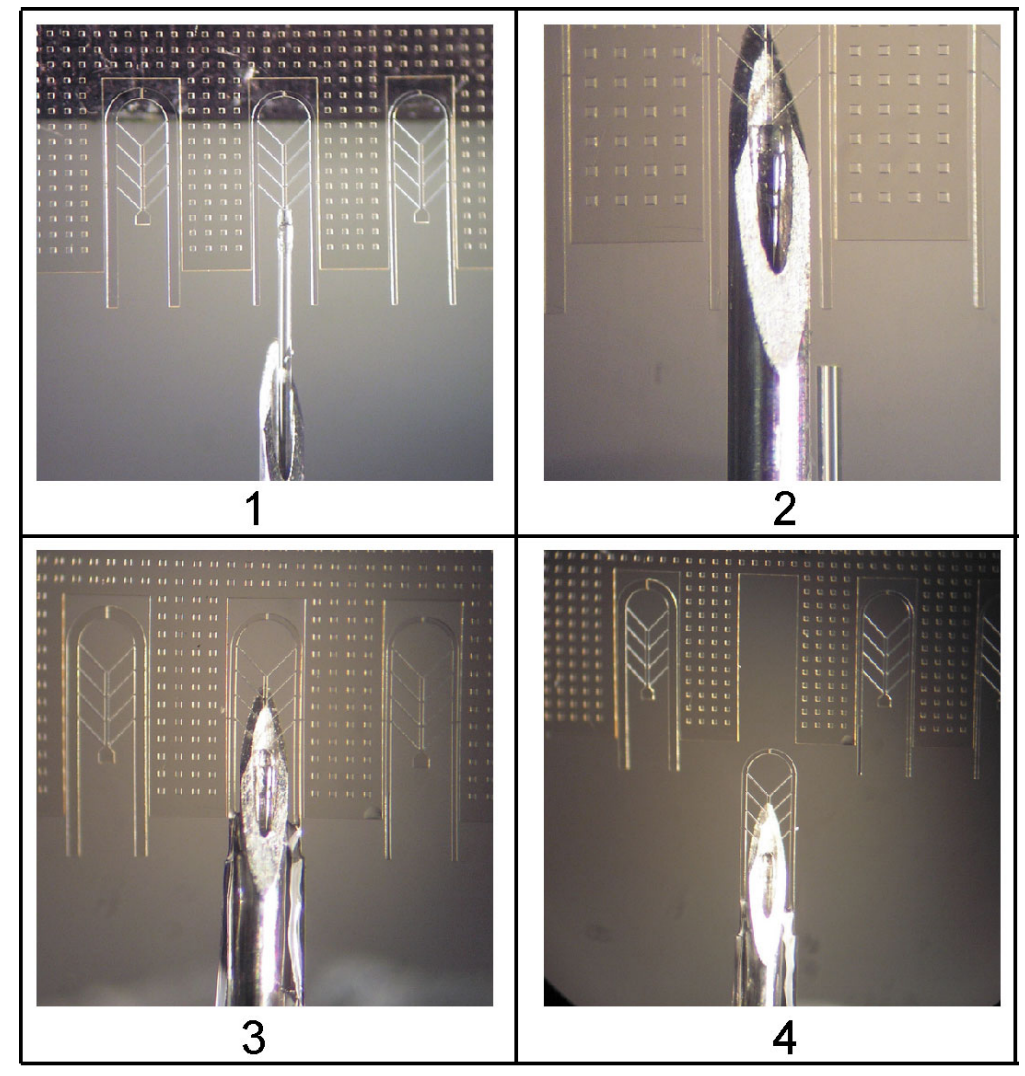

Figure 5.8. Assembly process sequence. 1) Shaft bonding to backbone, 2) first fiber alignment-bonding process, 3) second fiber is bonded with the same procedure, 4) Microgripper is detached from the main frame. 
The process of attaching the shaft to the backbone had a degree of difficulty which depended on the actuator of choice. As it can be observed from figure 5.8-1, initially the shaft is extended far out from the hollow cylinder to prevent the glue from bonding the shaft to the cylinder. In the case of using a micrometer screw as an actuator, the process only required bonding the actuator to the shaft, and using the actuator itself it was possible to extend the shaft far enough from the hollow cylinder allowing the backbone gluing process. The assembly using the piezoelectric actuator requires the positioning of the actuator in a precise and final location. In order to make the shaft-backbone bonding process possible, a micrometer screw is attached to the piezoelectric actuator (already bonded to the shaft), which is used to push the shaft far enough to allow gluing, and then used to pull back the microgripper (still in the frame). The piezoelectric actuator is then fixed in place, and the micrometer screw is then detached.

The assembly process, even though highly manual, was reliably performed for the $100 \mu \mathrm{m}$ and $50 \mu \mathrm{m}$ grippers, proving the production of these devices feasible. Assembly of microgrippers with narrower gripping arms proved to be more challenging, with a low success rate due to the difficulty of applying glue to such small structures. The results presented in the next chapter correspond to the $100 \mu \mathrm{m}$ and $50 \mu \mathrm{m}$ microgrippers. It should be mentioned that a more accurate, automated assembly process could be conceived with an accuracy better than $5 \mu \mathrm{m}$, which would allow the implementation of smaller microgrippers. 


\section{MECHANICAL CHARACTERIZATION AND RESULTS}

\subsection{Mechanical characterization}

As discussed in previous chapters, the microgrippers were actuated in two ways: first, in a purely mechanical manner by means of a micrometer screw; secondly, in a electromechanical manner using a piezoelectric actuator. The first technique of actuation has the advantage of allowing a large range of actuation, as well as the possibility to push and pull the backbone. The piezoelectric actuation provided the possibility for more elaborated characterization since it allowed cycling the microgrippers at a given frequency. Results from both techniques of actuation are presented in the following subsections.

\subsubsection{Micrometer screw actuation testing}

After a $50 \mu \mathrm{m}$ arm microgripper assembly process was completed, it was tested by turning the micrometer actuator knob to achieve different target opening gaps. The system was monitored using an Olympus MX40F optical microscope with a 100x objective and a COHU 2222-1320 CCD camera. The target gaps were a) $0 \mu \mathrm{m}$, b) $5 \mu \mathrm{m}$, c) $10 \mu \mathrm{m}$, d) $20 \mu \mathrm{m}$, e) $30 \mu \mathrm{m}$, and f) $40 \mu \mathrm{m}$. Figure 6.1 shows optical micrographs of the microgripper opened to desired gap dimensions. The target gap values were obtained within the resolution of the measuring system, except for the closed value of $0 \mu \mathrm{m}$, which was not clearly observed; the smallest gap observed was $2.4 \mu \mathrm{m}$. It is possible that the

gripping facets actually made contact at their bottom edge. This is believed to be due to the non-perfect verticality of the facets of the gripper, which implies that the facets had a positive profile of approximately 2 degrees, corresponding to $1.2 \mu \mathrm{m}$ over $30 \mu \mathrm{m}$ profile. 


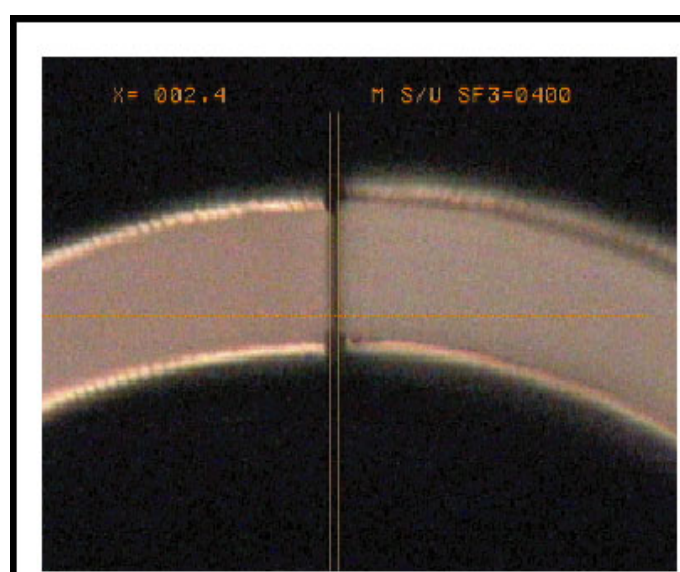

(a)

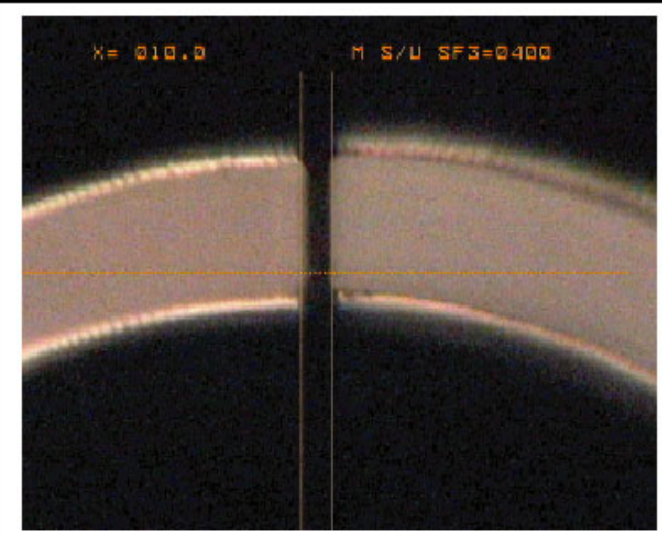

(c)

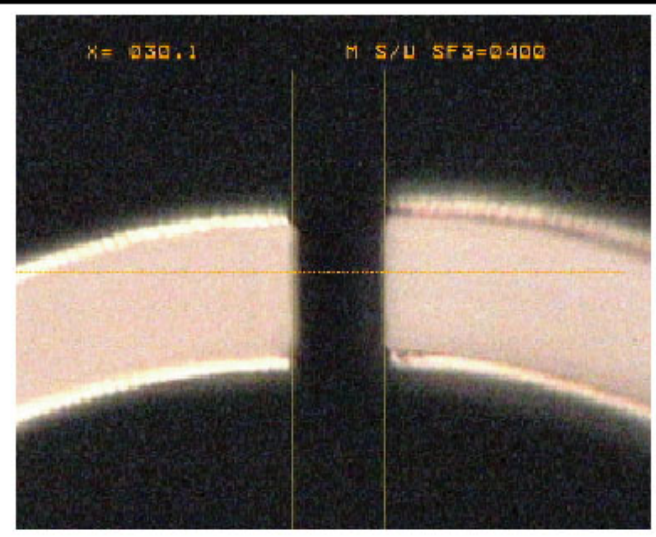

(e)

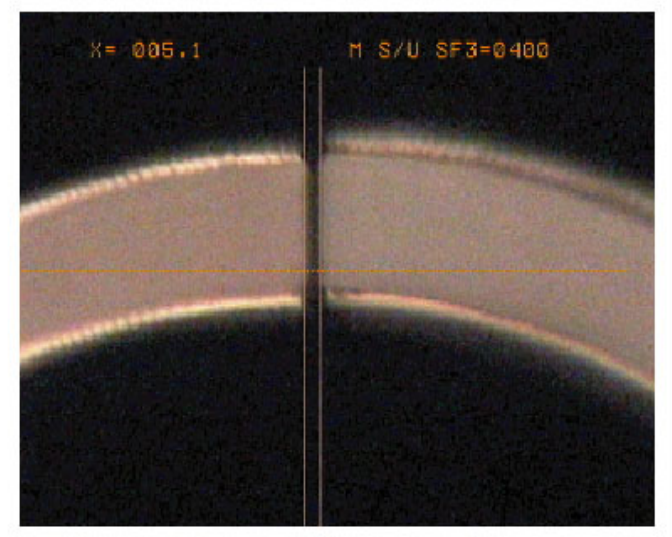

(b)

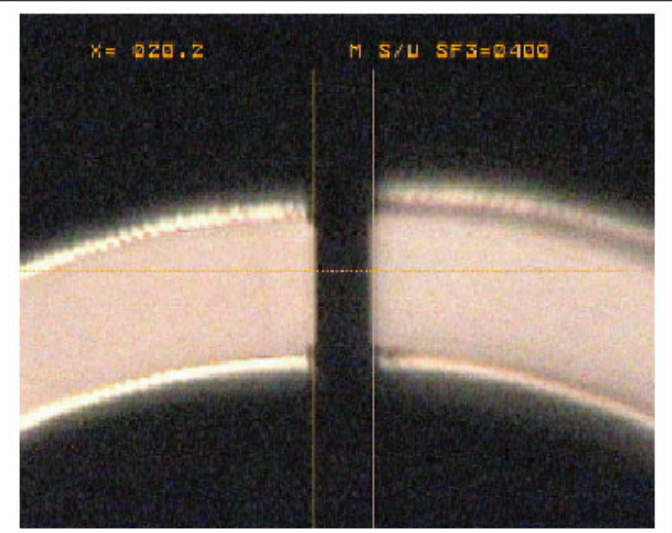

(d)

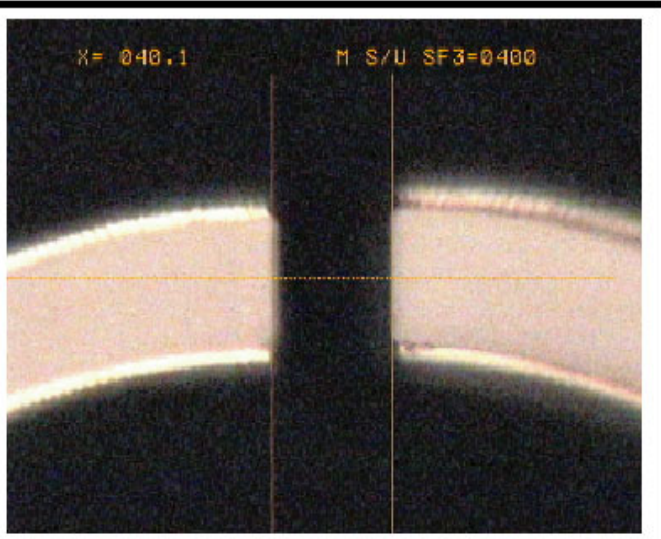

(f)

Figure 6.1. Microgripper (50 $\mu \mathrm{m}$ arm) actuated mechanically with a micrometer screw showing different levels of actuation. (a) $2.4 \mu \mathrm{m}$, (b) $5 \mu \mathrm{m}$, (c) $10 \mu \mathrm{m}$, (d) $20 \mu \mathrm{m}$, (e) $30 \mu \mathrm{m}$, and (f) $40 \mu \mathrm{m}$. 
In order to determine the maximum opening gap, the microgripper was further actuated, reaching successfully a $130 \mu \mathrm{m}$ gap, however when actuated up to $140 \mu \mathrm{m}$ fracture occurred at the "V" joints formed by the spines and both the microgripper arms as well as the backbone (see figure 6.2). It is expected that if the operation of the device is limited to openings below $100 \mu \mathrm{m}$ (approximately 25\% below its maximum achievable), the lifespan of the device will be longer than if the device would be operated in the $100-130 \mu \mathrm{m}$ range, however further experimentation is needed.

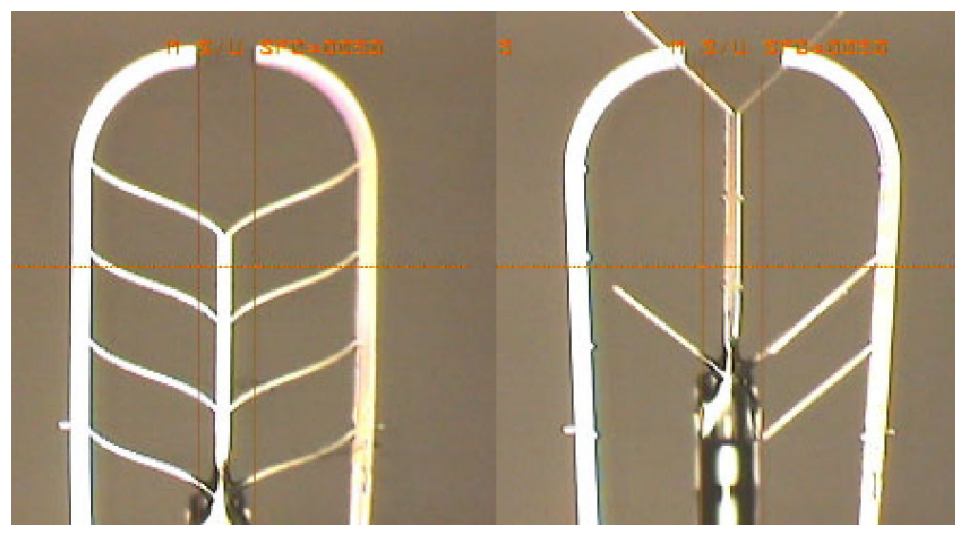

(a)

(b)

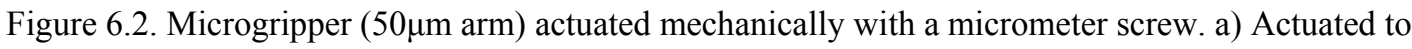
$\sim 130 \mu \mathrm{m}, \mathrm{b})$ Device failure when attempted to open to $140 \mu \mathrm{m}$, fractures occurred.

As discussed in Chapter IV, the "V" junctions are points where, upon actuation, stress is concentrated, so it is no surprise that fracture occurred at these points. However, a factor that could cause early fractures could be microcracks sometimes present at this junctions, as these can propagate. In a randomly chosen sample, it was observed that these microcracks were not present before the complete release of the structure from the substrate; however they were present after the release process. It is suspected that these microcracks could originate when the floating structures are removed from the BOE, immersed in DI water, and then removed from it. The physical interaction of two liquids, 
and the SU-8 structures, because of their surface tension, could cause forces that could originate these microcracks; however, for this reason to be confirmed, further investigation is required. The suspicions of the microcracks causing an early fracture could be investigated by finite element simulations, however, modeling a microcrack within a relatively large device would require a extremely fine mesh, which would require high computational power. For this reason, this phenomenon was not simulated. A microcrack at a "V" junction example is presented in figure 6.3 indicated by the white arrow.

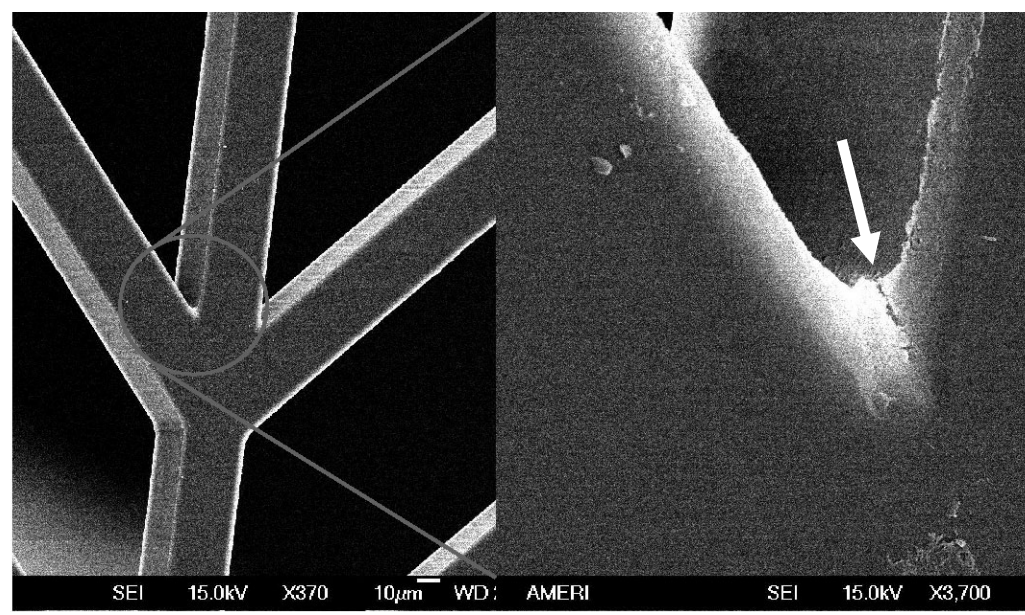

Figure 6.3. Microcrack present at the "V" junctions pointed by white arrow. Even though the device is expected to fracture at these points under extensive actuation, device failure (fracture) could happen prematurely at these points due to microcrack propagation.

\subsubsection{Piezoelectric actuation testing}

For electromechanical actuation, a piezoelectric stack actuator (Thorlabs part number AE0505D18) with $15 \mu \mathrm{m}$ range was used. This piezoactuator consists of several piezoelectric ceramic layers assembled mechanically in series and electrically in parallel. To drive the piezoelectric actuator, a General Photonics PZD001 piezo-controller was used. The system was tested utilizing the previously mentioned optical microscope and 
CCD camera. Gap openings were measured as the driving voltage was varied from 0 to 120 volts in steps of 10 volts. This was followed by measuring the gap as the voltage was decreased to 0 volts also in steps of 10 volts of approximately 10 seconds duration. Figure 6.4 shows the resulting gap versus actuation voltage plot for the ramp-up and ramp-down of the voltage across the piezoactuator for a $100 \mu \mathrm{m}$ wide arm microgripper.

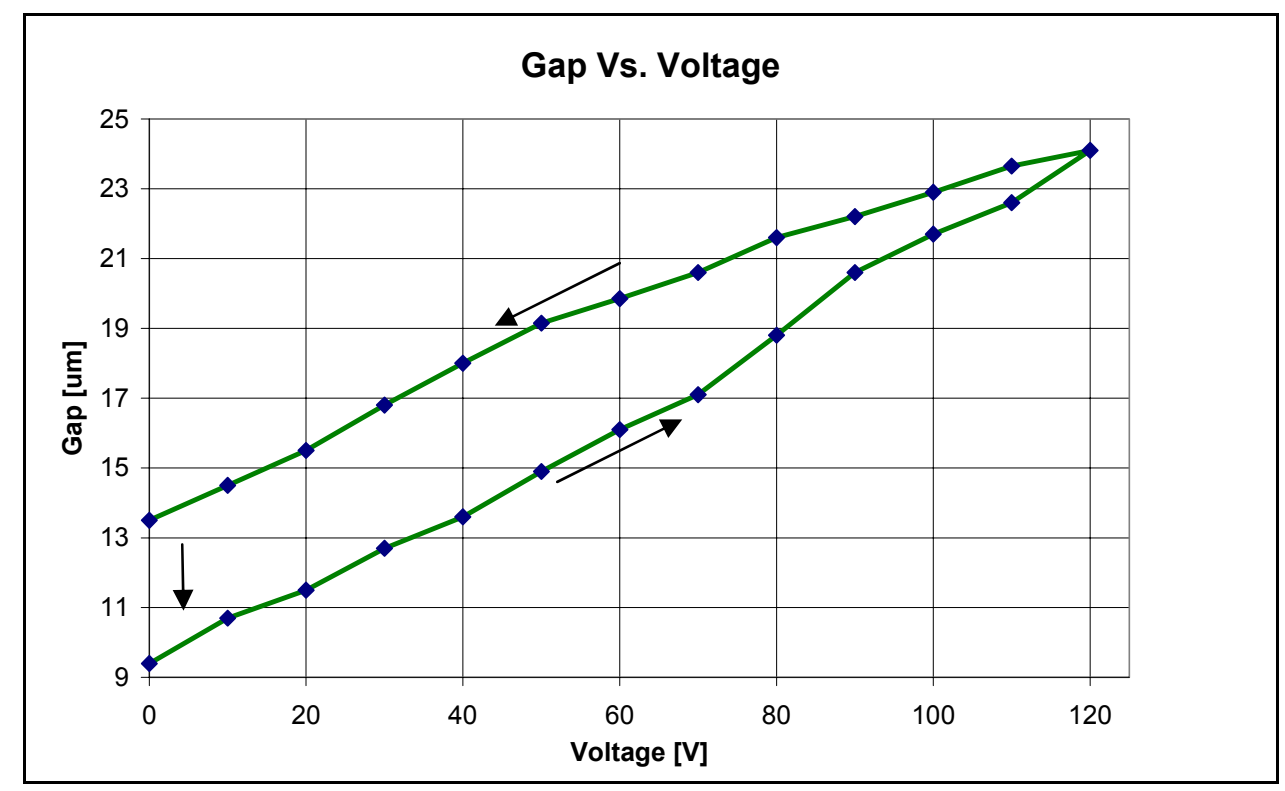

Figure 6.4. Measured microgripper gap $(100 \mu \mathrm{m}$ arm $)$ for different voltages. Voltage was increased in steps of 10 volts from 0 to 120 volts and then lowered back to 0 volts as indicated by the arrows showing hysteresis. After some time, the gap returns to the starting value.

Ideally, the gap vs. voltage curve should be retraced as the voltage is reduced back to zero, and therefore returning the gap to its original size. However, as the voltage is lowered to 0 volts, the curve takes a different direction, showing hysteresis. Hysteresis is commonly seen in piezoelectric actuators and is due to internal stresses stored inside the piezoelectric actuator which prevent the system to respond immediately. Hysteresis in the piezoactuator was accentuated because of the slow rate at which the measurements were taken, maintaining a DC voltage applied for a few seconds. It was also observed that when lowering the voltage to zero, after some time the gap value would return to the 
corresponding initial value. As mentioned in Chapter I one, it is believed that the hysteresis observed can be overcome by feedback from strain sensors, in a way that any nonlinearity due to hysteresis could be compensated in real time. Another possibility to overcome this drawback is to employ a nonlinear hysteresis model to estimate hysteresis effects and compensate this effects using feed-forward control schemes [23]. Finally, it should be noted that SU-8 polymers may also present hysteresis after substantial mechanical deformations, not approached in this experiment.

\subsection{Single cell manipulation}

The microgripper's micromechanical capabilities for single cell manipulation were demonstrated with SKOV-3 human ovarian carcinoma cells suspended in McCoy's 5A cell media with $10 \%$ (volume percent) fetal bovine serum (FBS) and 1\% (volume percent) streptomyocin. The objective of this experiment was to be able to isolate a single cell from a randomly distributed population of cells. The cells size ranged from 10 to $30 \mu \mathrm{m}$ approximately, so microgrippers with $50 \mu \mathrm{m}$ wide arms were used. The microgripper was mounted on a micromanipulator which was used to position it over a glass slide containing the cells.

The process was monitored by means of an Olympus IX81 motorized inverted microscope with a Qimaging Retiga EXi camera. The gripper had an angle of incidence with respect to the glass slide of approximately 45 degrees, which restricted focus to the complete microgripper at the same time. Figure 6.5. shows a sequence of steps where the device is used to successfully manipulate a single cell, as follows: 1) the microgripper is closed, 2) microgripper is opened by means of the piezoelectric actuator, 3) cell is 
approached by moving the stage supporting the glass slide and grasped, 4) the grasped cell is moved away and isolated. Similar results where obtained also with the $100 \mu \mathrm{m}$ wide arm microgripper.

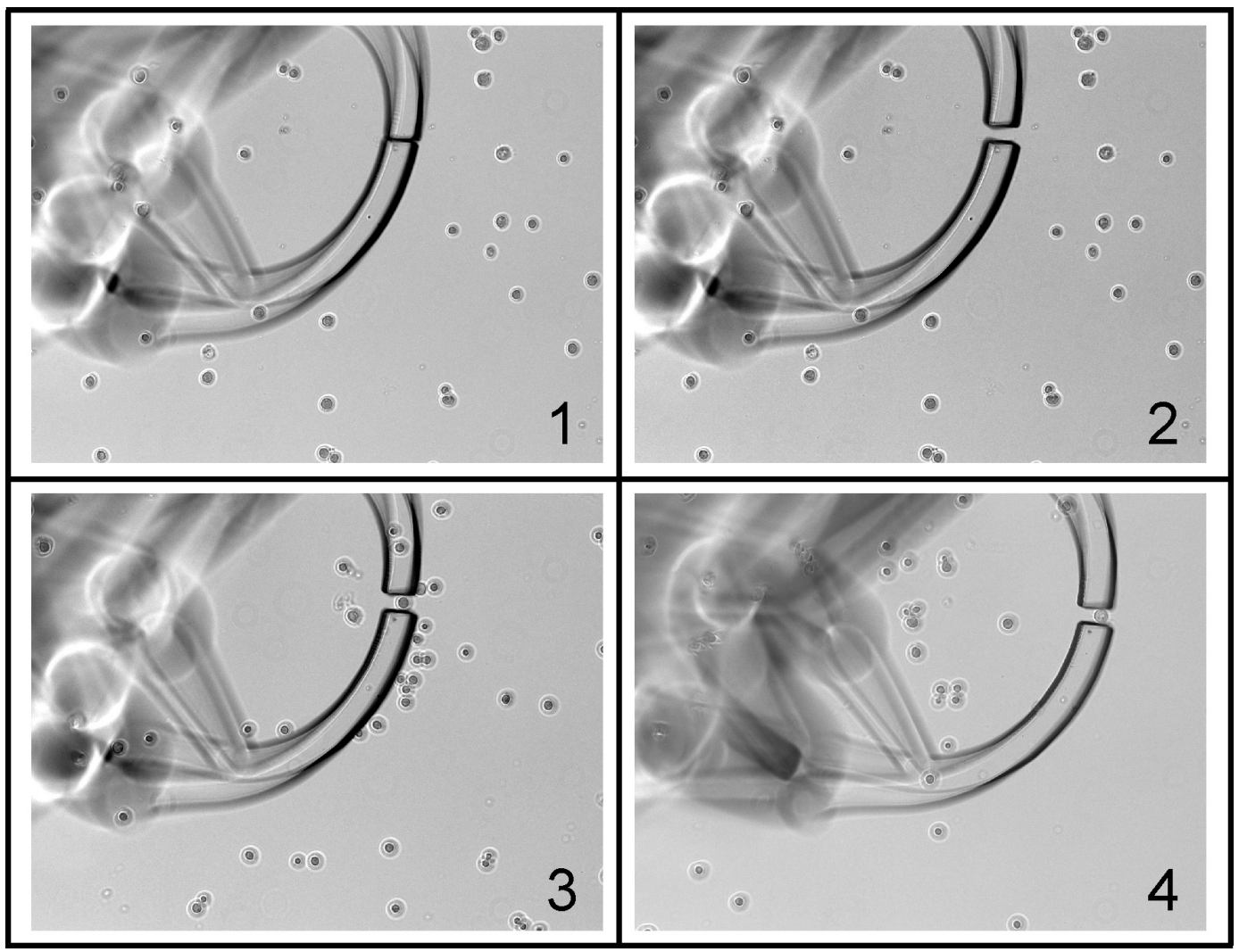

Figure 6.5. Manipulation of a single SKOV-3 cell utilizing a $50 \mu \mathrm{m}$ wide arm microgripper. 1) microgripper closed, 2) gripper opens, 3) cell is approached and grasped, 4) cell is moved and isolated. 


\section{OPTICAL CHARACTERIZATION AND RESULTS}

\subsection{Optical performance characterization}

Optical testing was performed for the $100 \mu \mathrm{m}$ and $50 \mu \mathrm{m}$ wide arm microgrippers due to ease of assembly. Light was coupled from a $128 \mathrm{~mW}, 532 \mathrm{~nm} \mathrm{Nd:YAG} \mathrm{laser} \mathrm{to} \mathrm{the}$ input fiber, while monitoring the output power by connecting the output fiber to a photo detector. Later, for fluorescence measurements, the output was connected to an optical spectrum analyzer (OSA), controlled by a computer for data collection. A system diagram is presented in figure 7.1.

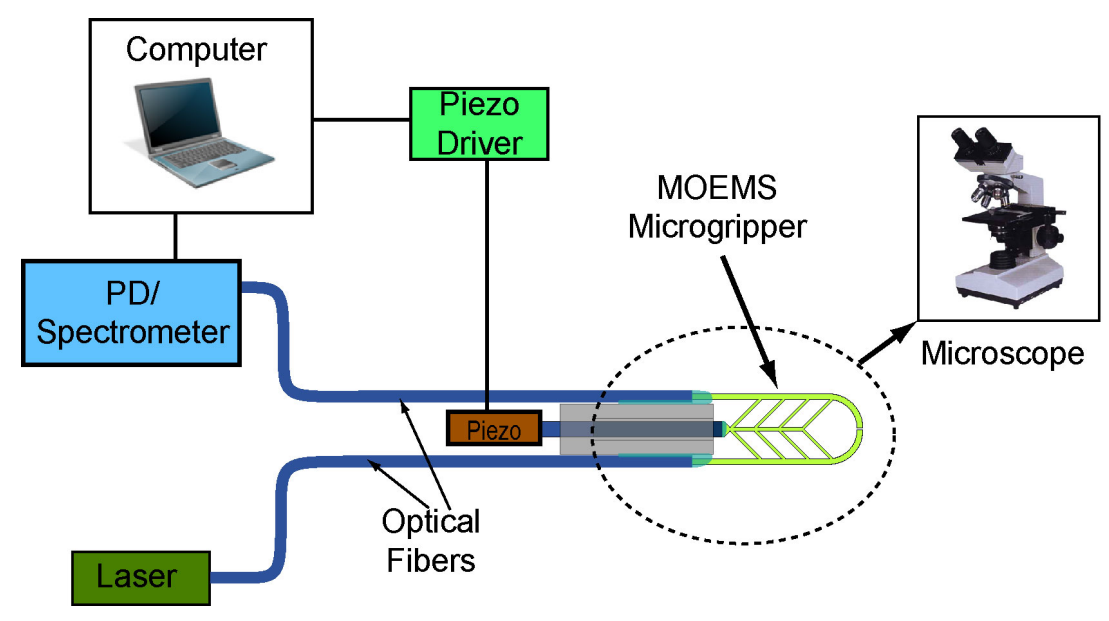

Figure 7.1. System diagram showing setup for MOEMS microgripper testing. Laser light is coupled to the microgripper by means of an optical fiber. The light coupled to through the facets is monitored by means of a photo detector or a spectrometer.

\subsubsection{Light-guiding demonstration}

Initially an SMF28 fiber with approximately $10 \mu \mathrm{m}$ core was used to couple light into the $100 \mu \mathrm{m}$ waveguide arm of the microgripper (figure 7.2-1a). This fiber is single mode for infrared, however it is multimode at $532 \mathrm{~nm}$, consequently, a large number of modes is excited in the $100 \mu \mathrm{m}$ waveguide. Ray-like propagation was observed in the waveguide, which can be observed in the micrograph presented in figure $7.2-1 \mathrm{~b}$, which 
shows the orange fluorescence of the SU-8 material (wide band fluorescence centered at $560 \mathrm{~nm}$ [31]) excited by the propagating rays; this was observed through a filter that eliminates $532 \mathrm{~nm}$. This ray-like propagation was predicted by optical simulations (Chapter IV). Figure 7.3 presents a close-up of figure 7.2-1b near the facets, which resembles its corresponding optical simulation for which the first 10 modes of the waveguide were launched. A significant improvement in coupling efficiency was obtained when a $50 \mu \mathrm{m}$ multimode fiber was used to couple light into a $50 \mu \mathrm{m}$ width waveguide, allowing a better matching of modes and uniform illumination (figure 7.2-2a). Figure 7.2-2b shows the corresponding SU-8 fluorescence.

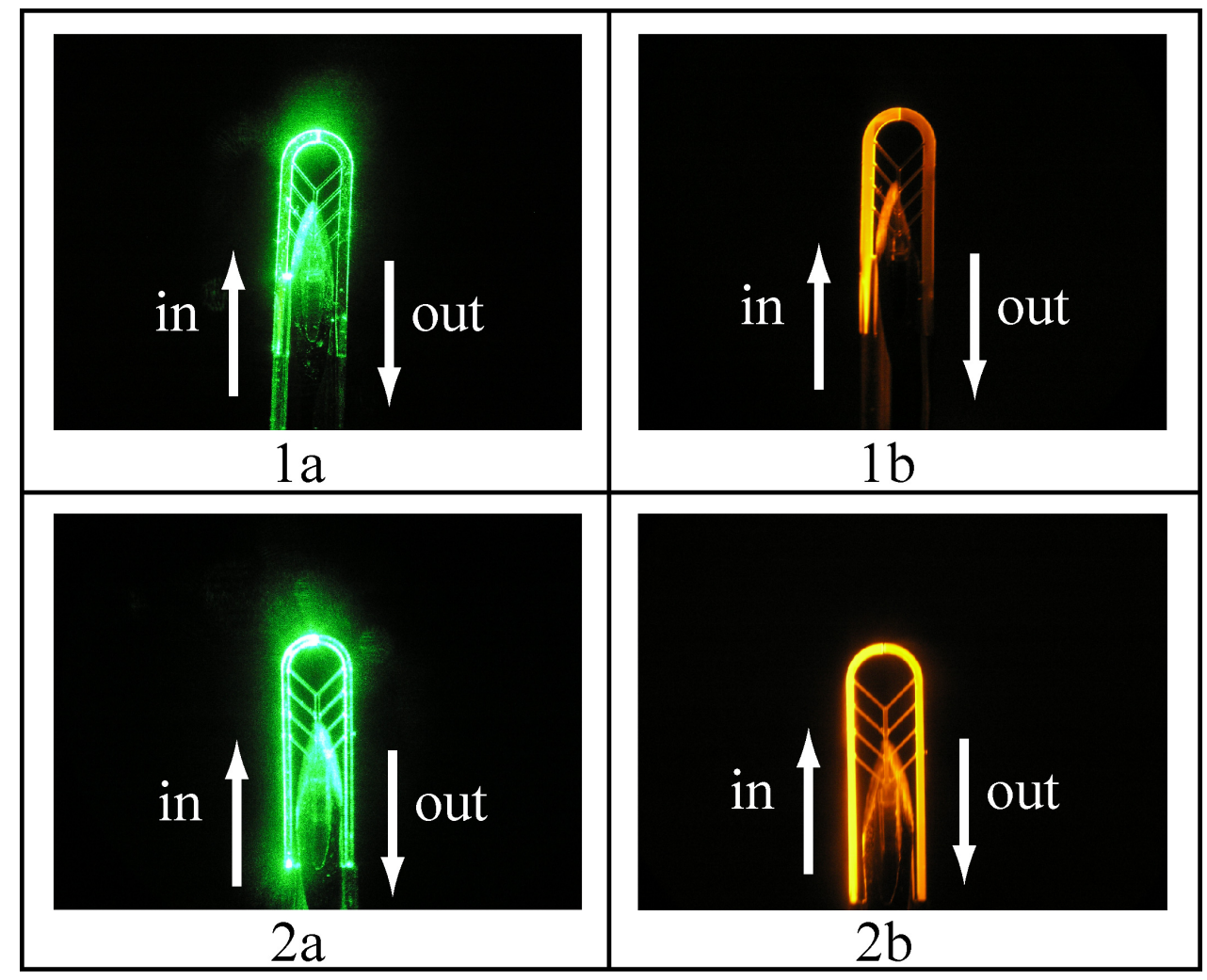

Figure 7.2. Coupling light from a $128 \mathrm{~mW}, 532 \mathrm{~nm}$ laser to the input fiber. Output power was monitored by connecting the output fiber to a photo-detector. 1a) SMF28 fiber with approximately $10 \mu \mathrm{m}$ core was used to couple light into the $100 \mu \mathrm{m}$ waveguide arm of the microgripper. $2 \mathrm{a}$ ) $50 \mu \mathrm{m}$ multimode fiber was used to couple light into a $50 \mu \mathrm{m}$ waveguide arm, allowing a better matching of modes and uniform illumination.

Figures $1 \mathrm{~b}$ and $2 \mathrm{~b}$ are $1 \mathrm{a}$ and $1 \mathrm{~b}$ under a filter that eliminates $532 \mathrm{~nm}$, showing the fluorescence of the SU-8 material. 


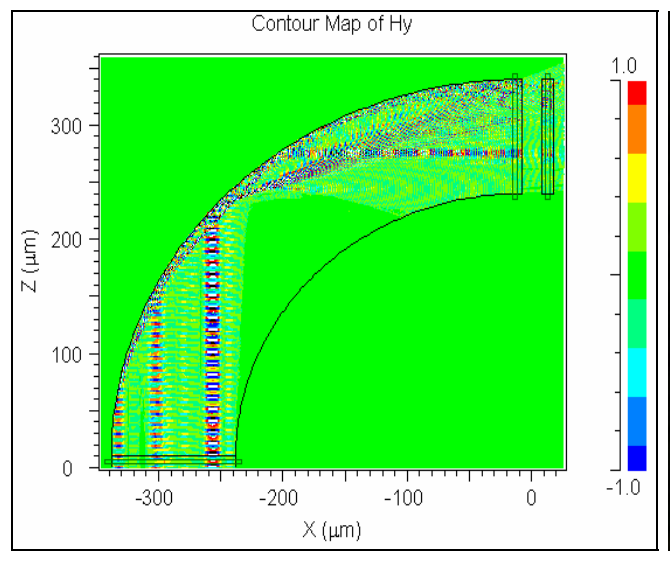

(a)

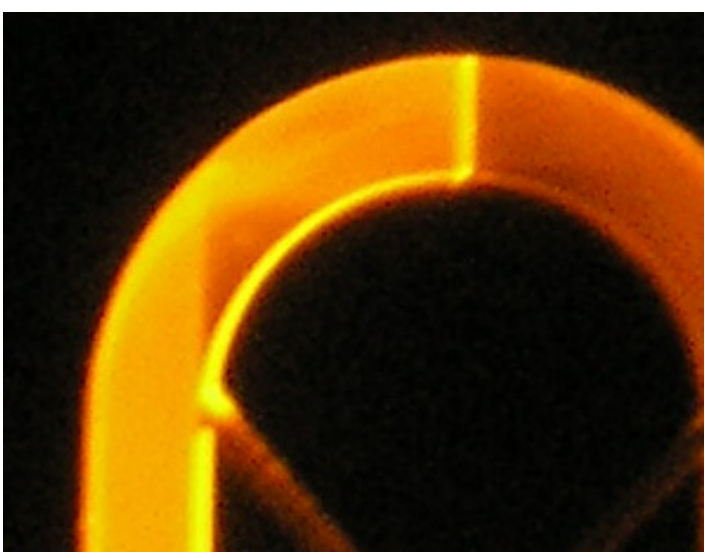

(b)

Figure 7.3. Comparison between simulated and observed light propagation at the $100 \mu \mathrm{m}$ waveguide bends:

a) Simulation from launching the first 10 modes, b) Experimental observation of modes reflecting at the polymer-air interface, notice ray-like propagation which resembles the simulation.

From figure 7.2 it is noticeable that there is a significant amount of light leaking out of the waveguides, specially scattered through the sidewalls due to their roughness.

Figure 7.4 shows a SEM micrograph of a section of SU-8 at the corner of these optical waveguides in which the sidewall roughness is noticeable. While the top of the waveguide is smooth due to the fact that SU-8 is spin-coated, the sidewall roughness is due to the photolithographic fabrication process, because the sidewalls are chemically

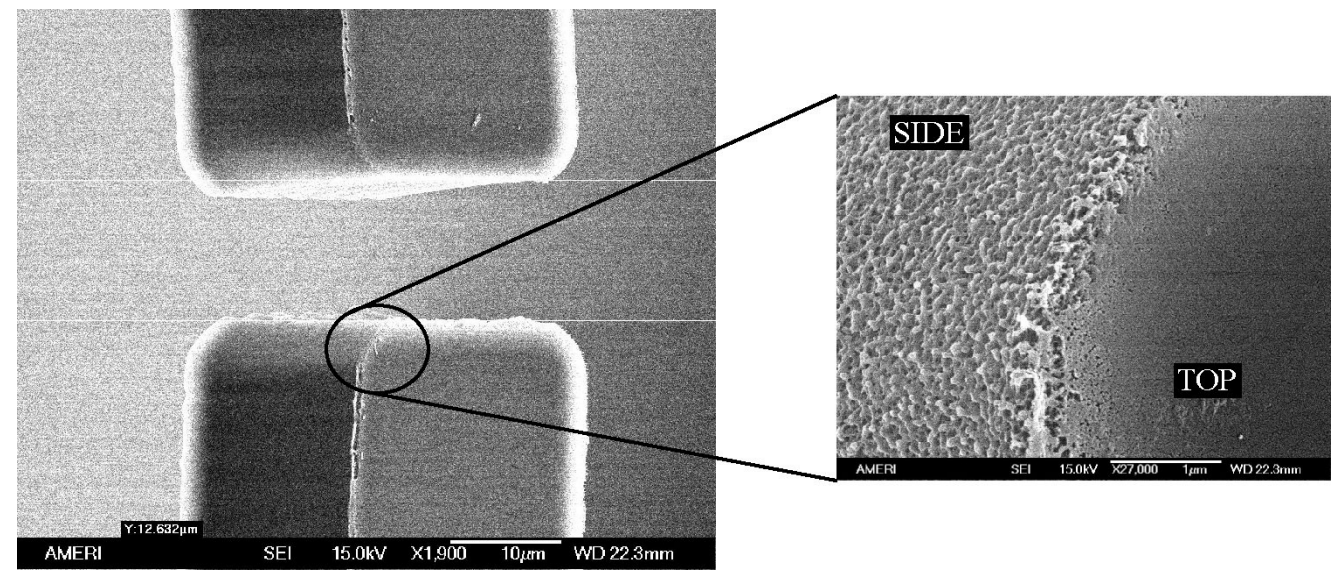

Figure 7.4. SEM Micrograph showing a section of SU-8 optical waveguide. The top smoothness comes from spin-coating the polymer, while the side roughness is due to the photolithographic fabrication process which chemically defines the sidewalls. 
defined by the developer (PGMEA). Sidewall roughness can be mitigated by performing a further optimization of the photolithographic process, furthermore, the use of a more sophisticated fabrication technique, such as proton beam writing [13] would significantly minimize this roughness; however, this technique is recommended for prototyping rather than mass production.

\subsubsection{Microstructure detection-binary feedback}

One of the simplest capabilities of the microgrippers is to be able to detect the presence of a microobject between the microgripper facets by monitoring the intensity of light coupled between the facets. A simple binary feedback can be obtained given that the intensity of coupled light drops when there is an object blocking the light. Figure 7.5 shows on the left the microgripper with nothing between the facets, as light couples from the left to the right waveguide; and on the right the microgrippers grasping a $20 \mu \mathrm{m}$ thick aluminum slab; notice that light does not couple to the right waveguide. It was observed that the slab between the facets causes a reduction of $83 \%$ in the power detected at the microgripper output. It should be mentioned that, even though light does not couple through the facets, there is still some power detected at the output of the microgripper. This power corresponds to a fraction of the scattered light from the left waveguide which couples to the waveguide on the right. Also, from figure 7.4, it can be noticed that since the object between the facets is reflective, not only does it block the light, but also it reflects it back in to the waveguide (notice the increase in illumination on the input waveguide), being this an indication that the binary feedback can be implemented not only in transmission, but also in reflection mode with the use of an optical circulator. 


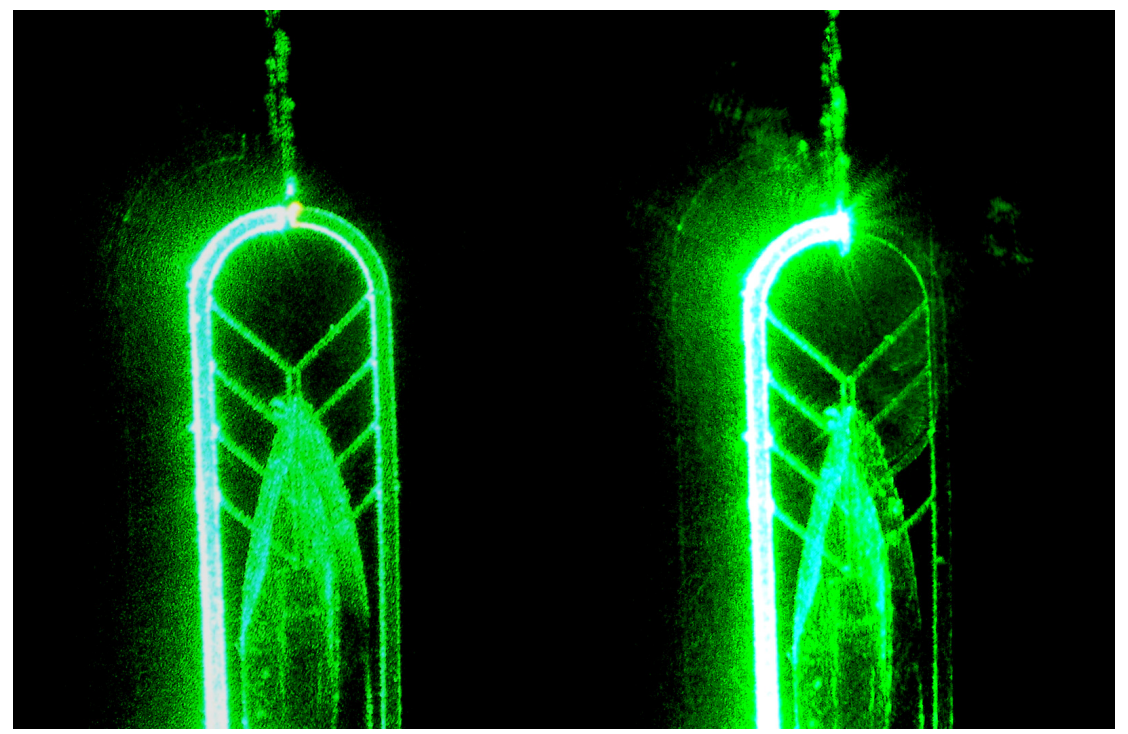

(a)

(b)

Figure 7.5. Microobject detection with binary feedback. a) the microgripper with nothing between the facets, where light is coupled from the left to the right arm, and b) the microgrippers grasping a $20 \mu \mathrm{m}$ thick aluminum slab. Notice the significant reduction of light in the left arm, with corresponding increase in illumination at the input arm do to the high reflectivity of the slab.

\subsubsection{Optical power coupled between facets}

To determine the effect of the distance between the microgripper facets on the total power coupled between them, the following experiment was executed. While coupling laser light at $532 \mathrm{~nm}$ to the input fiber of a $50 \mu \mathrm{m}$ arm microgripper, the optical power transmitted through the microgripper gap was monitored by measuring the power present at the end of the output fiber. For this, a Newport $1835 \mathrm{c}$ multifunction optical meter was utilized, while the piezo-electric actuator was driven with a triangular signal varying from $0 \mathrm{~V}$ up to $45 \mathrm{~V}$ which corresponds to varying the gap from $16 \mu \mathrm{m}$ (relaxed microgripper gap) to approximately $21 \mu \mathrm{m}(\sim 5 \mu \mathrm{m}$ increase). Figure 7.6 shows the normalized measured intensity of the transmitted optical power as the microgripper opens. From figure 7.6, it can be observed that as the microgripper facets separate, the power coupled between the facets is modulated, presenting a series of peaks. 


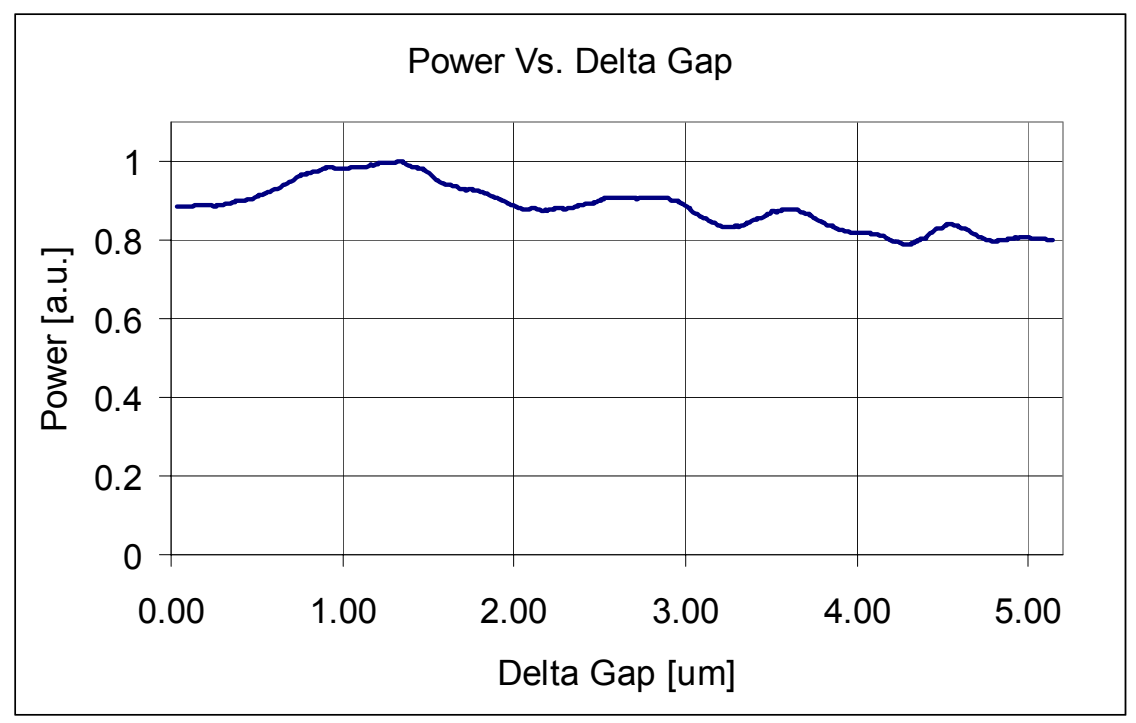

Figure 7.6. Optical power monitored at the output of the microgripper's opening is increased from its initial state by $\sim 5 \mu \mathrm{m}$.

Considering that the facets form a SU-8 - air - SU-8 cavity, it is worth investigating the possibility of Fabry-Perot (FP) resonances being related to the modulation observed. The transmitted light through a FP cavity is given by [24]:

$$
I_{\text {transmited }}=I_{\text {incident }} \frac{(1-R)^{2}}{(1-R)^{2}+4 \cdot R \cdot \sin ^{2}\left(\frac{2 \pi}{\lambda} L\right)}
$$

Where $R$ is the reflectance at the walls of the cavity, and $\mathrm{L}$ is the length of the cavity. From equation 7.1 , the transmitted power will be maximum for cavity length multiples of $\lambda / 2$. The peaks observed in the transmitted as the microgripper gap is varied, (figure 7.6) appear approximately every $2 \lambda$, which clearly shows that the modulation observed does not come from pure FP resonances. Nevertheless, a modulation intensity in transmission on the order of 20 percent was observed, similar to that of a FP cavity with a 5\% reflectance (from equation 7.1, where $R$ is 0.05 calculated from equation 2.10 for SU-8 and air) due to index of refraction contrast; figure 7.7 presents the expected transmission 


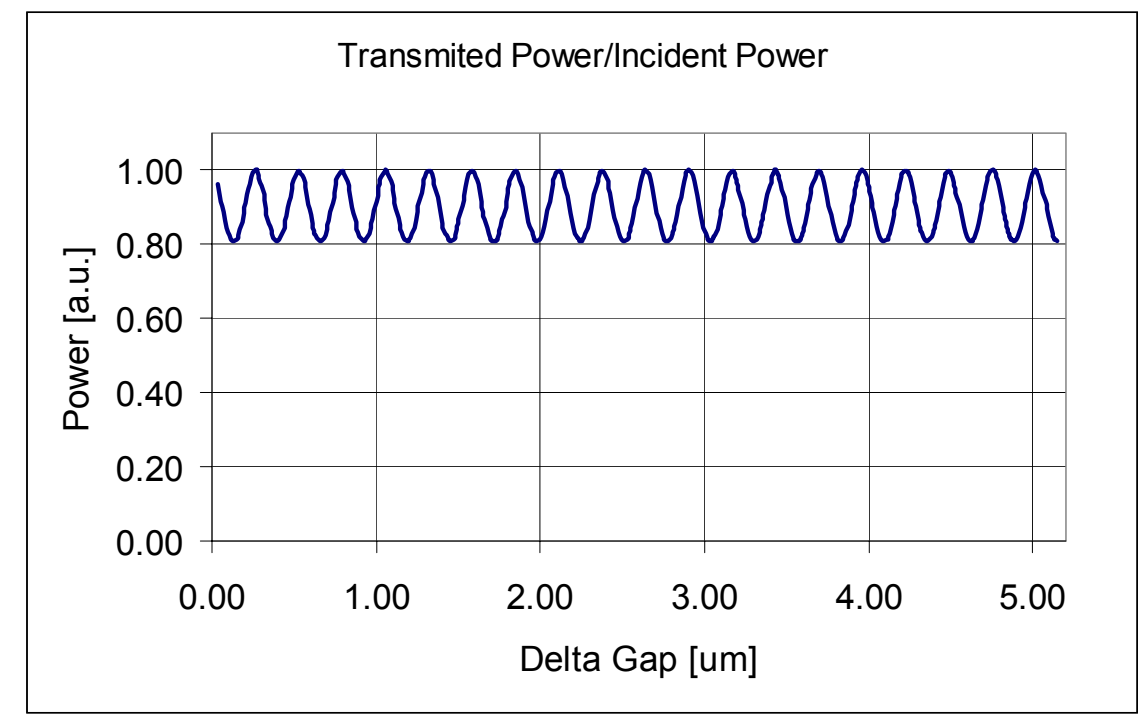

Figure 7.7. Calculated transmitted power at a wavelength of $532 \mathrm{~nm}$ for a SU-8 - air - SU-8 cavity as the cavity length is increased by $5 \mu \mathrm{m}$

of power at a wavelength of $532 \mathrm{~nm}$ for a SU-8 - air - SU-8. The factors leading to the observed transmission modulation are not certainly known, however, factors that could contribute to this phenomenon could be the non-perfectly vertical facets, coupled with the multimodality of the structure, which could produce constructive and destructive interference as the microgripper opens.

\subsubsection{Frequency response}

Depending on the application given to the MOEMS microgrippers, high speed operation may be required, for example, automated assembly of 3D-MEMS in a mass production environment. For this, it is important to determine their frequency response. Measuring the microgrippers frequency response was greatly facilitated by the ability of the microgrippers to carry light, and the fact that optical power is modulated as it couples from facet to facet (figure 7.6). To measure the frequency response, a 7265 DSP Lock-in amplifier from SIGNAL RECOVERY was utilized. The Lock-in amplifier's reference 
signal was utilized to control the piezo driver in a way that the actuation frequency would be swept along a given range. The light collected from the output gripping arm was monitored with a Newport $1835 \mathrm{c}$ power meter, which at the time produced an equivalent analog signal, which was fed back to the lock-in amplifier. The reference signal was swept from $10 \mathrm{~Hz}$ up to $1 \mathrm{KHz}$. The response measured from a $50 \mu \mathrm{m}$ arm microgripper is presented in figure 7.8. It was observed that the microgrippers $-3 \mathrm{~dB}$ frequency is approximately $500 \mathrm{~Hz}$. It is worth mentioning that this frequency response corresponds to the complete system, which besides the SU-8 structure, includes the shaft and the piezoactuator. The $-3 \mathrm{~dB}$ frequency of the SU-8 structure alone is estimated to be $\sim 8 \mathrm{kHz}$.

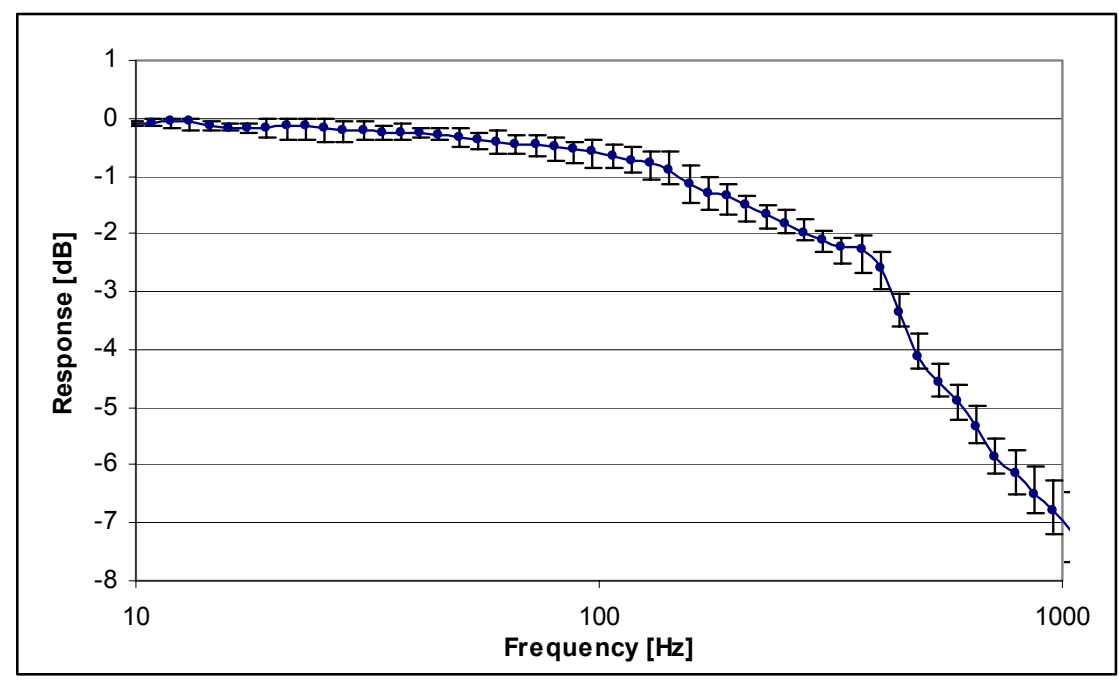

Figure 7.8. Frequency response from $50 \mu \mathrm{m}$ arm microgripper measured by monitoring the coupled light modulation induced by varying the gap between the facets (opening and closing the microgripper).

The $-3 \mathrm{~dB}$ frequency is approximately $500 \mathrm{~Hz}$.

\subsubsection{Step response}

A second important parameter for an application requiring high speed operation is to know how long it takes for the microgripper to respond to a step signal, or in other words, the step response. For the MOEMS microgrippers, this parameter can be extracted 
from the variation of the coupled power across the facets in response to a step voltage applied to the piezo. Figure 7.9 presents four normalized curves corresponding to the power coupled across the facets in response to steps different magnitudes. The time it takes for the optical power coupled to drop from $10 \%$ to $90 \%$ was measured to be $1.6 \mathrm{~ms}$ for the 30 Volts step (incrementing the gap by $2 \mu \mathrm{m}$ ) up to $2.5 \mathrm{~ms}$ for the 120 volts step (incrementing the gap by $14 \mu \mathrm{m}$ ).

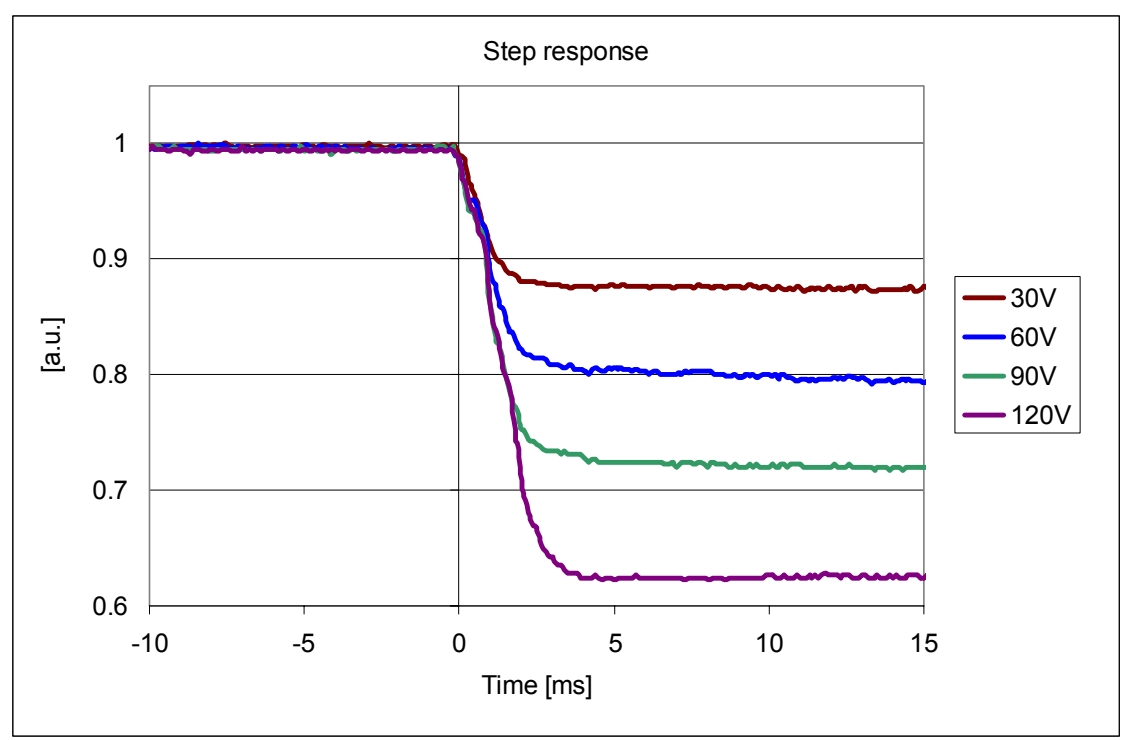

Figure 7.9. Normalized curves corresponding to the power coupled across the facets in response to steps of different magnitudes. The time it takes for the optical power coupled to drop from $10 \%$ to $90 \%$ was measured to be $1.6 \mathrm{~ms}$ for the 30 Volts step (incrementing the gap by $2 \mu \mathrm{m}$ ) and $2.5 \mathrm{~ms}$ for the 120 volts step (incrementing the gap by $14 \mu \mathrm{m}$ ).

\subsection{Quantum dot tagged micro parts identification}

The ability of carrying light to and from the microobject being held by the microgrippers makes it possible to identify microparts tagged with a fluorescent agent. In this experiment, three $30 \mu \mathrm{m}$ thick SU-8 slabs were coated with $2 \mu \mathrm{l}$ of quantum dots (QDs) in solution over an area of $1.5 \mathrm{~mm}$ by $10 \mathrm{~mm}$, and let dry. QDs with three different emission wavelengths: $555 \mathrm{~nm}, 599 \mathrm{~nm}, 612 \mathrm{~nm}$ were utilized, one kind per slab. The 
QDs were suspended in toluene, with a concentration in $\mathrm{nmol} / \mathrm{ml}$ of $57.17,21.29$, and 17.85 respectively. Green laser light $(532 \mathrm{~nm})$ was coupled to the fiber connected to the microgripper's input to excite the QDs. The SU-8 slabs were grasped by opening the microgripper, placing the slabs between the gripping facets, and closing the microgripper.

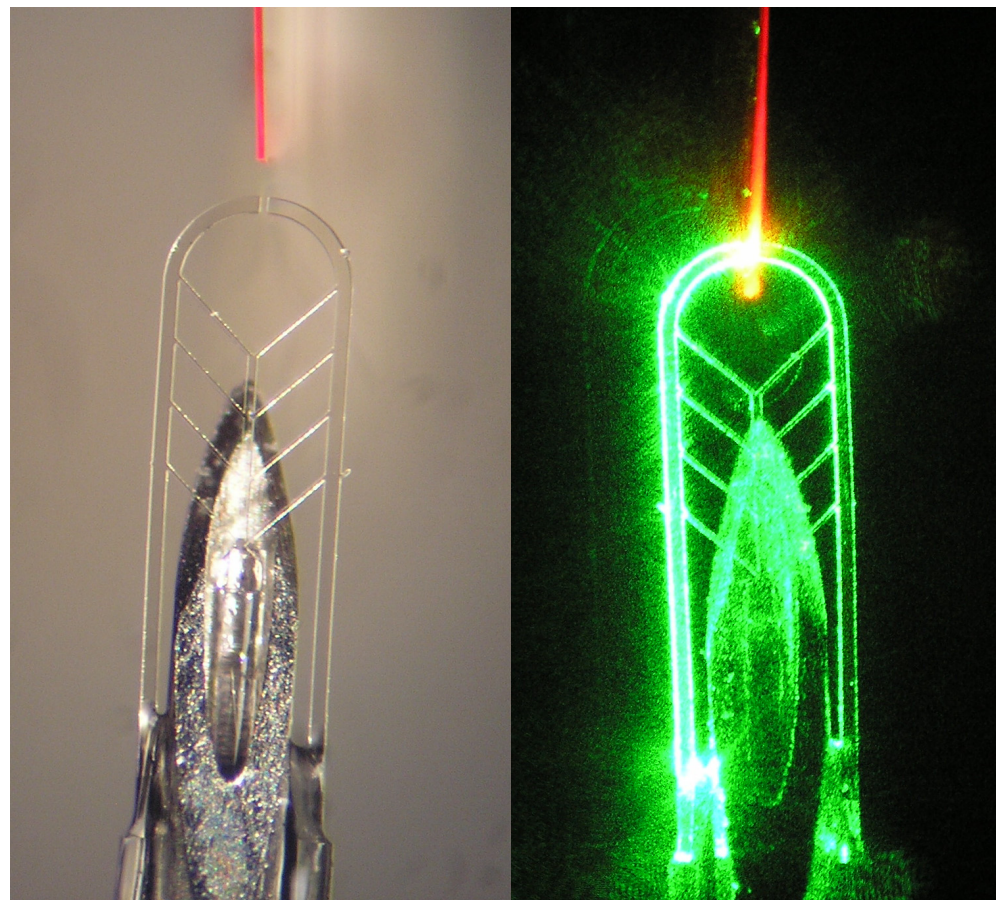

(a)

(b)

Figure 7.10. Micropart identification through fluorescence detection. a) Microgripper approaching a QD tagged SU-8 slab with (laser off), b) Microgripper grasping the SU-8 slab, collecting the emitted fluorescence.

The QDs tagged slabs were therefore illuminated through the waveguides with the green laser, and consequently fluoresced in their corresponding emission wavelength. This florescent light is collected by the output facet/arm which is connected through a fiber to a USB2000 Newport optical spectrum analyzer (OSA). Figure 7.10 presents, on the left, the microgripper approaching a QD tagged SU-8 slab with the laser off, and on the right, the microgripper grasping the SU-8 slab where the fluorescence of the QDs is clearly observed, which is then collected by the second facet. 
It should be mentioned that the second facet not only collects the fluorescence from the QDs, but also it collects the green light from the laser that is transmitted through the SU-8 slab. The laser light was filtered out using a $550 \mathrm{~nm}$ long pass filter, which allowed only the fluorescent light to reach the OSA. Figure 7.11 presents the emission collected from the three different QD tagged SU-8 slabs, with a 500 ms integration time. The asymmetry of the peak corresponding to $555 \mathrm{~nm}$ is due to its closeness to the long pass cutoff wavelength of $550 \mathrm{~nm}$. Notice that the three peaks can be clearly identified.

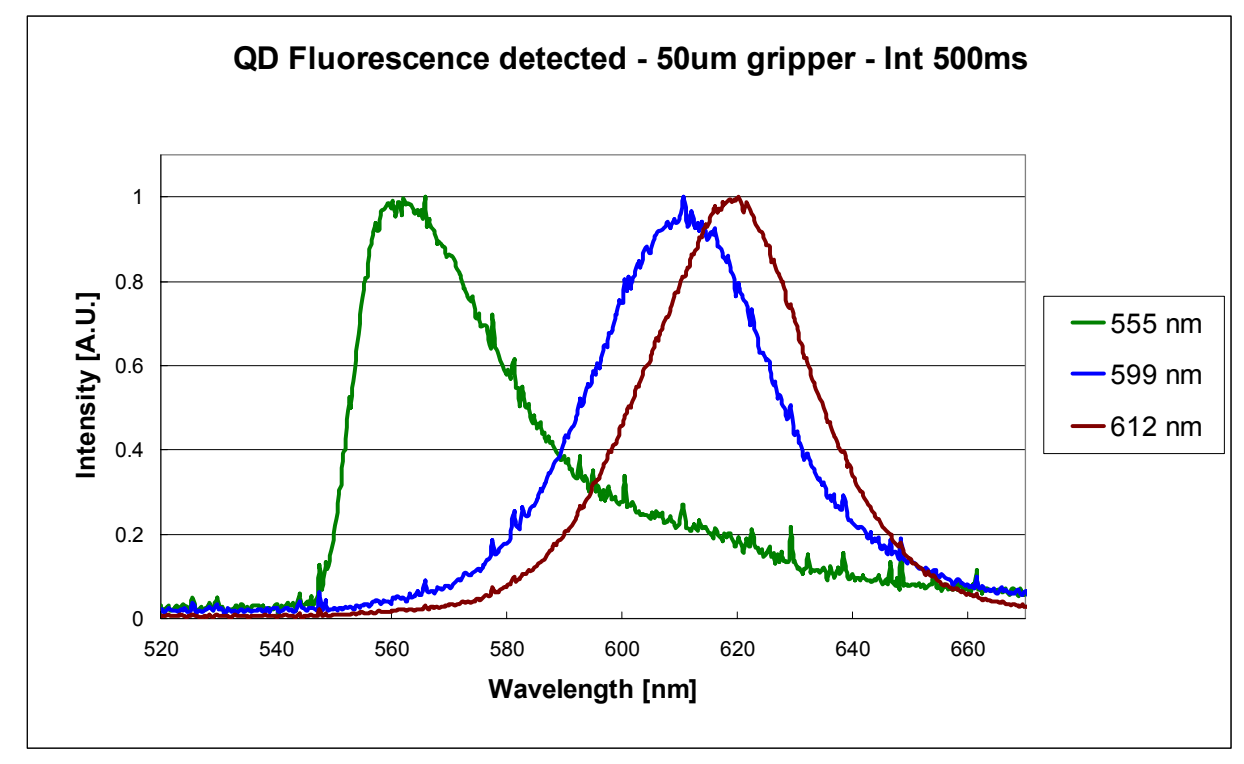

Figure 7.11. Fluorescence detected from SU-8 microparts tagged with QDs with emission at 555, 599, and $612 \mathrm{~nm}$ with an integration time of $500 \mathrm{~ms}$.

Fluorescence measurements were taken with 50,100, 500, $1000 \mathrm{~ms}$ integration time, and it was observed that a significant signal to noise ratio was detected even with the shortest integration times. Figure 7.12 presents the measurements taken for the slab tagged with $599 \mathrm{~nm}$ QDs with the different integration times. The strength of the fluorescence signal is directly proportional to the concentration of QDs present in the micropart. In the case of having a low QD concentration tag, the weakness of the signal 
could be compensated by adjusting the photodetector integration time. Also, as previously discussed, the acceptance angle fore a SU-8 waveguide suspended in air is $180^{\circ}$, which is highly beneficial for efficiently collecting the fluorescence emitted by the QDs.

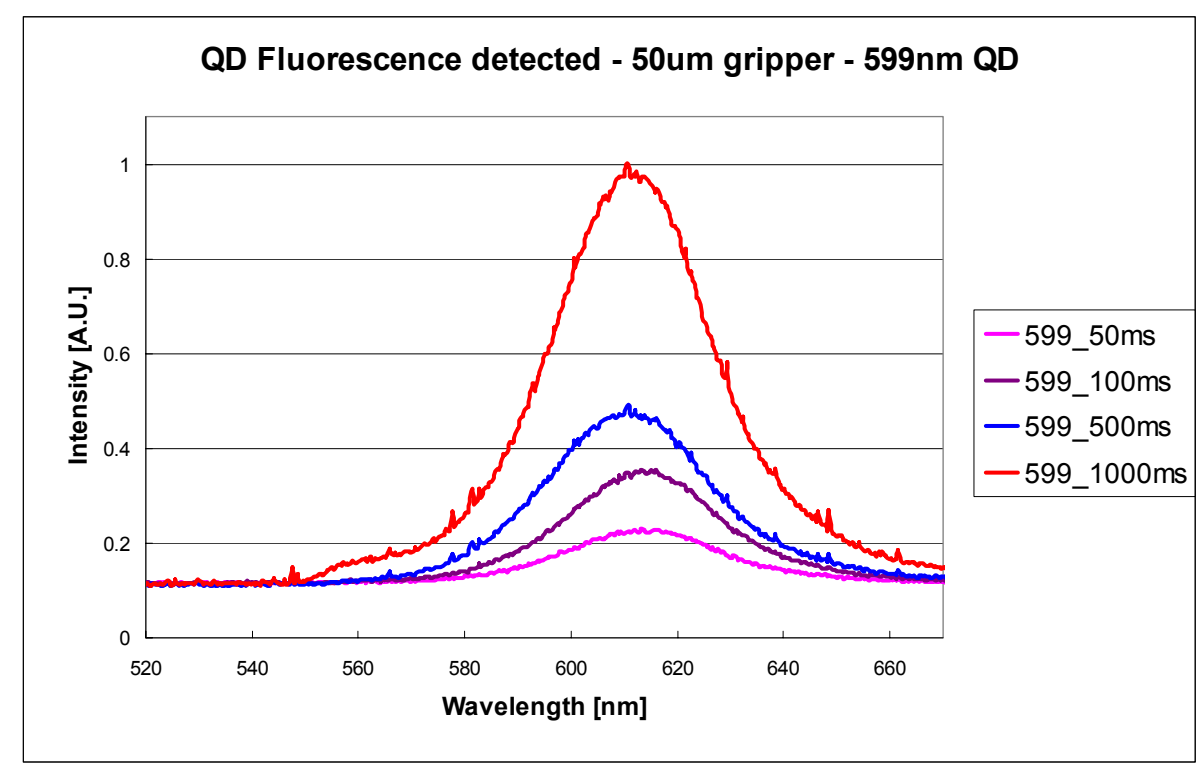

Figure 7.12. Fluorescence detected from a single SU-8 micropart tagged with QDs with emission at $599 \mathrm{~nm}$ with integration times ranging from $50 \mathrm{~ms}$ up to $1000 \mathrm{~ms}$. Notice that even at the shortest integration times, there is still a high signal to noise ratio. 


\section{SUMMARY, CONCLUSIONS, AND FUTURE WORK}

\subsection{Summary and conclusions}

In this research work, novel Micro-Opto-Electro-Mechanical SU-8 microgrippers with integrated optical waveguides have been successfully demonstrated. The development process started with a thorough review on existing microgrippers. From literature it was determined that several of the microgrippers developed so far by other researchers have no integrated feedback, and a few have a limited number feedback mechanisms. This was followed by providing the necessary background of MEMS and optical waveguides, to provide the foundation of the presented work.

The microgrippers developed in this work are composed of flexible optical waveguides, and a fishbone actuation mechanism, which requires an external actuator for it to open and close the microgrippers. The fishbone maintains the facets parallel within 1 degree, facilitating optical characterization as well as mechanical handling. The optical waveguides also perform as gripping arms, providing the physical means for grasping a microobject, as well as enabling light to be delivered and also collected in transmission, reflection or fluorescent mode, thus allowing the optical characterization of the structure under study, facilitated by the high $N A$ of the gripping facets. The design process was described, and the proposed devices functionality was verified through optical and mechanical simulations. The microgrippers were fabricated completely in house by means of a simple fabrication process flow, and characterized mechanically and optically, using two kinds of external actuators: a micrometer screw, and a piezoelectric actuator. It 
was determined that the microgrippers have the potential to manipulate structures from submicron dimensions up to $100 \mu \mathrm{m}$.

The system implementation was accomplished through a manual assembly process as a proof of concept for microgrippers of this kind. This assembly technique allowed to assemble the microgrippers with gripping arms widths of $50 \mu \mathrm{m}$ and $100 \mu \mathrm{m}$ in a consistent manner, while the microgrippers with narrower gripping arms assembly proved to be more challenging, with a low success rate given that they are less mechanically robust than the wider waveguide microgrippers. It is estimated that a completely automated assembly process could be easily conceived requiring an accuracy better than $5 \mu \mathrm{m}$, which would allow the implementation of more delicate microgrippers reliably. An interesting observation that was made during the device characterization was that varying the gap dimension causes a noticeable modulation in the power detected at the output of the microgripper. Even though the exact reasons for this are not known, it is suspected that the multimodality of the structure plays a significant role, along with nonperfectly vertical facets. This optical modulation facilitated the mechanical response characterization of the microgrippers.

Frequency response was measured for the $50 \mu \mathrm{m}$ arm microgrippers for which a $-3 \mathrm{~dB}$ frequency of $500 \mathrm{~Hz}$ was determined; however, this response corresponds to the complete system (SU-8 structure, shaft, piezoactuator) operating together, and not the SU-8 structure alone, which is expected to have a frequency response of $\sim 8 \mathrm{KHz}$. The step response was measured to be $1.6 \mathrm{~ms}$ for a $30 \mathrm{~V}$ step which increases the gap by $2 \mu \mathrm{m}$, and $2.5 \mathrm{~ms}$ for a 120 volts step corresponding to incrementing the gap between the facets of $14 \mu \mathrm{m}$. The mechanical functionality of the microgrippers was demonstrated 
with a practical application which consisted in manipulating single SKOV-3 human ovarian carcinoma cells which ranged in size between $10 \mu \mathrm{m}$ to $30 \mu \mathrm{m}$. Single cell manipulation was accomplished using $50 \mu \mathrm{m}$ and $100 \mu \mathrm{m}$ wide arm microgrippers.

Optical binary feedback capabilities to detect the presence of a microobject between the gripping facets were demonstrated in transmission mode by observing a reduction of $83 \%$ in coupled light from the input to the output waveguide when there is a microobject between the facets, and in reflection mode by detecting the illumination increase in the input waveguide due to the light being reflected from the microobject.

The detection and identification of microparts tagged with a fluorescent "barcode" was demonstrated. The barcode was implemented with quantum dots, with emission wavelengths of $555 \mathrm{~nm}, 599 \mathrm{~nm}, 612 \mathrm{~nm}$. Fluorescence from the QDs was exited though laser light at $532 \mathrm{~nm}$. The microparts were clearly differentiated from each other by detecting the fluorescence of their corresponding QDs, or fluorescent barcode. Fluorescence was detected with integration times from $1000 \mathrm{~ms}$ down to $50 \mathrm{~ms}$. A significant signal to noise ratio in the fluorescence peaks was observed even with the shortest integration time.

The novel MOEMS microgrippers presented open up new possibilities in the field of micromanipulation for the microscopic study of biological entities such as cells tagged with fluorescent agents, as well as an improvement in 3D MEMS assembly, with a more sophisticated optical feedback mechanism which allows fast detection and identification of microparts. Another application were the MOEMS microgrippers could contribute is flow cytometry where microscopic particles (such as biological cells) tagged with a fluorescent agent are counted and sorted while suspended in a stream of fluid. 
The microgrippers presented are scalable to the nano domain, which expands their possible applications. Nano-grippers could be implemented in SU-8 as well as in silicon. It should be mentioned that silicon geometries such as optical ring resonators with radii of $2 \mu \mathrm{m}$, and $450 \mathrm{~nm}$ waveguides have already been successfully implemented in the Nanophotonics Research Lab at FIU, providing this the necessary expertise to implement in the future microgrippers in Silicon in the submicron range.

To the best of the author's knowledge, the presented devices are the first functional Micro-Opto-Electro-Mechanical (MOEMS) microgrippers with an integrated feedback mechanism implemented in SU-8.

\subsection{Future work}

The presented work represents a significant milestone for the development of the micromanipulation field, given that MOEMS microgrippers are the first of their kind. Having demonstrated the fundamental properties and capabilities of the microgrippers, it is important to pursue further research, in a way that the full potential of MOEMS microgrippers can be achieved. The following subsections present different future tasks that could be pursued to further understand the devices, as well as to broaden their fields of application.

\subsubsection{Tapered facets}

Assembling microgrippers with $50 \mu \mathrm{m}$ and $100 \mu \mathrm{m}$ arms was successfully accomplished, however the assembly of microgrippers with narrower arms proved to be more challenging. It would be desirable to assemble microgrippers with narrower arms to be able to manipulate smaller microobjets. The micro-gripping end-effector can be 
designed with a tapered waveguides leading to a reduced facet width down to single digit micron dimensions by simple contact photolithography. Further reductions are possible with more sophisticated projection lithography. This way, the microgrippers will be mechanically robust, however capable of grasping smaller microobjects with more precision. It is clear that the optical properties of the microgripper with tapered waveguides should be first optimized by means of simulations. An XP800 FEI focused ion beam system is available at FIU, which will allow to taper the waveguides even to submicron dimensions in SU-8 and also silicon. Figure 8.1 presents a conceptual diagram of a $50 \mu \mathrm{m}$ arm microgripper with tapered gripping arms down to $10 \mu \mathrm{m}$.

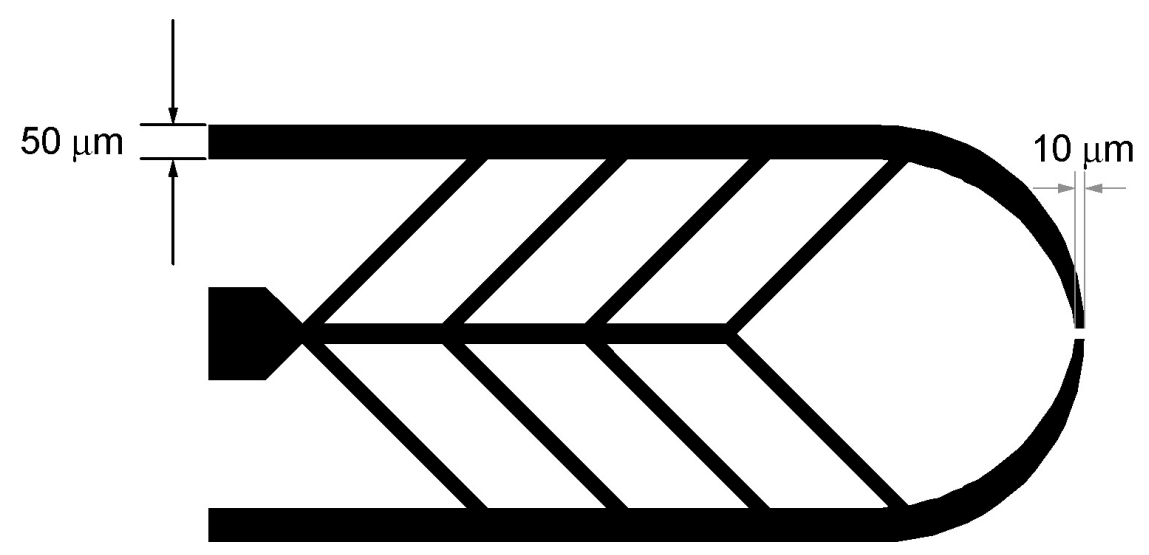

Figure 8.1. Conceptual diagram of a $50 \mu \mathrm{m}$ arm microgripper with tapered gripping arms to produce $10 \mu \mathrm{m}$ wide facets.

\subsubsection{Fluorescence detection optimization}

A remarkable result obtained with the microgrippers presented in this work is the capability of detecting fluorescence from a microobject between the facets. The microparts used for the demonstration of fluorescence detection contained a relatively high concentration of QDs, producing a strong signal. However, different microobjects, such as biological cells could be tagged with different fluorophores at low concentrations, 
which would emit a weaker signal. To optimize the fluorescence collected, the facet from the input arm could be modified to include a curvature, where a metallic aperture would be implemented. This aperture would reflect fluorescent light from the microobject, couple it into the receiving facet, and consequently optimize the signal strength. The trade-off of blocking some of the light to reach the microobject could be mitigated by placing the aperture in a region within the facet where the light intensity is maximum. The curvature in one of the facets would also facilitate the manipulation of round microobjects. A conceptual diagram of this implementation is presented in figure 8.2.

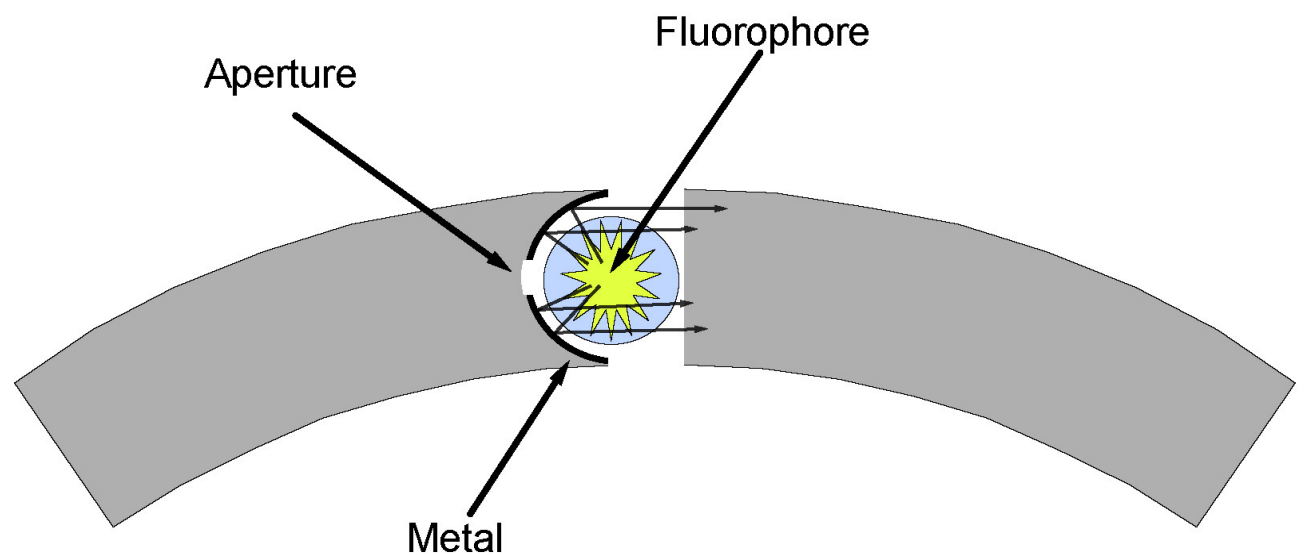

Figure 8.2. Conceptual diagram showing a modified facet to include a curved metallic aperture which would optimize the fluorescence coupled to the receiving facet.

\subsubsection{Force sensing}

So far, microgrippers with force sensing capabilities have been developed by means of interdigitated capacitor force sensors, semiconductor strain-gauges, among others. The fact that the microgrippers presented in this work utilize optical waveguides as gripping arms, make it possible to accomplish a simple force sensing structure by means of a Bragg grating patterned near one of the gripping arms bends. When the microgripper would grasp a microobject, the period of the grating would change, 
producing a shift in the reflected spectrum. This implies that that a circulator would be required to couple the laser required for the microgripper operation, and a tunable source for force sensing. A conceptual diagram of this implementation is presented in figure 8.3.

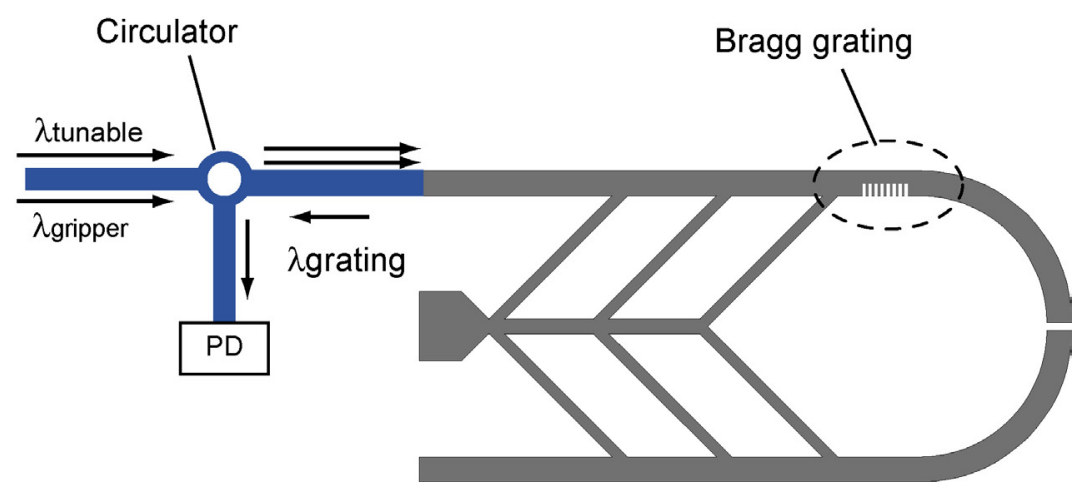

Figure 8.3. Conceptual diagram implementation of force sensing. A Bragg grating is patterned on one of the microgripper's arms. A circulator is utilized to couple the laser required for the microgripper operation, and a tunable source for force sensing. 


\section{REFERENCES}

[1] H.-S. Choi, D.-C. Lee, S.-S. Kim, and C.-S. Han, "The development of a microgripper with a perturbation-based configuration design method," Journal Of Micromechanics and Microengineering, vol. 15, p. 7, 16 May 2005.

[2] I. Roch, P. Bidaud, D. Collard, and L. Buchaillot, "Fabrication and Characterization of an SU-8 gripper actuated by a shape memory allow thin film," Journal of Micromechanics and Microengineering, p. 7, 2003.

[3] M. C. Carrozza, A. Eisinberg, A. Menciassi, D. Campolo, S. Micera, and P. Dario, "Towards a force-controlled microgripper for assembling biomedical microdevices," Journal of Micromechanics and Microengineering, vol. 10, p. 6, 2000 .

[4] M. Honnatti, G. Hughes, C. K., and J. B. Lee, "Directed cellular manipulation using polymer microgrippers," Zyvex application note 9720, 2006.

[5] N. Chronis and L. P. Lee, "Electrothermally Activated SU-8 Microgripper for Single Cell Manipulation in Solution," IEEE Journal of Microelectromechanical Systems, vol. 14, p. 7, August 2005.

[6] F. Beyeler, A. Neild, S. Oberti, D. J. Bell, Yu Sun, J. Dual, and B. J. Nelson, "Monolithically Fabricated Microgripper With Integrated Force Sensor for Manipulating Microobjects and Biological Cells Aligned in an Ultrasonic Field," IEEE Journal of Microelectromechanical Systems, vol. 16, p. 8, February 2007.

[7] N.-T. Nguyen, S.-S. Ho, and C. L.-N. Low, "A polymeric microgripper with integrated thermal actuators," Journal of Micromechanics and Microengineering, vol. 14, p. 5, May 202004.

[8] N. Dechev, W. L. Cleghorn, and J. K. Mills, "Microassembly of 3-D MEMS Structures Utilizing a MEMS Microgripper with a Robotic Manipulator," in IEEE International Conference on Robotics \& Automation, Taipei, Tsiusn, 2003.

[9] J. Park and W. Moon, "A hybrid-type micro-gripper with an integrated force sensor," Microsystem Technologies, vol. 9, p. 7, October 2003.

[10] M. S.-C. Lu, Z.-H. Wu, C.-E. Huang, S.-J. Hung, M.-H. Chen, and Y.-C. King, "CMOS micromachined grippers with on-chip optical detection," Journal of Micromechanics and Microengineering, vol. 17, p. 7, February 6, 2007.

[11] C. T. Lima, E. H. Zhoua, and S. T. Quekb, "Mechanical models for living cells - a review," Journal of Biomechanics, vol. 39 p. 21, 2006. 
[12] E. Conradie and D. Moore, "SU-8 thick photoresist processing as a functional material for MEMS applications," Journal of Micromechanics and Microengineering, p. 7, 13 June 2002.

[13] T. C. Sum, A. A. Bettiol, J. A. v. Kan, F. Watt, E. Y. B. Pun, and K. K. Tung, "Proton beam writing of low-loss polymer optical waveguides," Applied Physics Letters, vol. 83, p. 3, September 12003.

[14] MicroChem, "NanoTM SU-8 datasheet," Available on line at: http://www.microchem.com/products/pdf/SU8 2-25.pdf.

[15] R. P. Feynman, "There's plenty of room at the bottom," reprinted in $J$. Microelectromechanical Systems, vol. 2, p. 6, 1992.

[16] H. J. D. L. Santos, Introduction to microelectromechanical (MEM) microwave systems: Artech House, 1999.

[17] R. P. Feynman, "Infinitesimal Machinery," reprinted in $J$. Microelectromechanical Systems, vol. 1, 1993, p. 10, February 231983.

[18] C. Liu, Fundations of MEMS. New Jersey: Pearson Education, Inc., 2006.

[19] S. A. Campbell, The science and engineering of microelectronic fabrication. New York: Oxford University Press, 2001.

[20] G. S. May and S. M. Sze, Fundamentals of semiconductor fabrication: John Wiley \& Sons, Inc., 2004.

[21] M. J. Madou, Fundamentals of Microfabrication: The science of miniaturization, second ed.: CRC Press, 2002.

[22] D. Croft, G. Shedd, and S. Devasia, "Creep, hysteresis, and vibration compensation for piezoactuators: atomic force microscopy application," in American Control Conference, 2000, pp. 2123-2128.

[23] S. B. Choi, S. S. Han, and Y. S. Lee2, "Fine motion control of a moving stage using a piezoactuator associated with a displacement amplifier," Journal of Smart Materials and Structures, vol. 14, p. 9, 2005.

[24] S. O. Kasap, Optoelectronic devices and photonics: principles and practices. New Jersey: Prentice-Hall, Inc., 2001.

[25] J. C. Palais, Fiber optic comunications, Fifth ed. New Jersey: Pearson Education, Inc., 2005.

[26] G. Keiser, Optical fiber comuinications, Second ed.: McGraw-Hill, 1991. 
[27] I. Papakonstantinou, K. Wang, D. R. Selviah, and F. A. Fernandez, "Transition, radiation and propagation loss in polymer multimode waveguide bends," Optics Express, vol. 15, p. 10, January 22, 2007.

[28] J. P. Mills, L. Qie, M. Dao, C. T. Lim, and S. Suresh, "Nonlinear Elastic and Viscoelastic Deformation of the Human Red Blood Cell with Optical Tweezers," Molecular \& Cellular Biomechanics vol. 1, p. 11, 2004.

[29] J. A. Martinez and R. R. Panepucci, "Design, Fabrication, and Characterization of a Microgripper Device," in Florida Conference on Recent Advances in Robotics, FCRAR 2007 Tampa FL, 2007.

[30] J. A. Martinez, T. Liu, and R. R. Panepucci, "Micro-opto-electro-mechanical system (MOEMS) for microstructure manipulation and optical characterization," in SPIE 2007, San diego, California, 2007, p. 9.

[31] J. Hsieh, C.-J. Weng, H.-L. Yin, H.-H. Lin, and H.-Y. Chou, "Realization and characterization of SU-8 micro cylindrical lenses for in-plane micro optical systems," Microsystem Technologies, vol. 11, p. 8, 2005. 
VITA

\section{JOSE ANTONIO MARTINEZ}

January 18,1978

1999

2002

2004

$2002-2005$

$2005-2006$

2006 to present
Born, La Paz - Bolivia

A.A., Electrical Engineering

Miami Dade Community College

Miami, Florida

B.S., Electrical Engineering

Florida International University

Miami, Florida

M.S., Electrical Engineering

Florida International University

Miami, Florida

Outstanding Master's student award

Research Assistant

Future Aerospace Science \& Technology Center for Space Cryoelectronics

Florida International University

Miami, Florida

Process Engineer Co-op

Texas Instruments Inc. - DLP

Dallas, Texas

Research Assistant

Nanophotonics Research Group

Florida International University

Miami, Florida

\section{PUBLICATIONS AND PRESENTATIONS}

Main Author of "Micro-opto-electro-mechanical system (MOEMS) for microstructure manipulation and optical characterization" Proc. SPIE Int. Soc. Opt. Eng. 6645, 664525, 2007.

Main Author of "Design, Fabrication, and Characterization of a Microgripper Device," Proc. Florida Conference on Recent Advances in Robotics FCRAR 2007, Tampa, Florida, 2007. 
Main Author of "Design, simulation, and fabrication of a MEMs switched superconducting microstrip hairpin filter," Physica C: Superconductivity, Volume 466, Issues 1-2, 1 November 2007, Pages 101-105.

Co-author of "Wavelength Reconfigurable Photonic Switching Using Thermally Tuned Microring Resonators Fabricated on silicon substrate" Proc. SPIE Int. Soc. Opt. Eng. 6645, 66450I, 2007.

Co-author of "1x4 Wavelength Reconfigurable Photonic Switch Using Thermally Tuned Microring Resonators Fabricated on Silicon Substrate", IEEE Photonics Technol. Lett. 19, 704-706 MAY 1, 2007.

Coauthor of " $8 \times 8$ Wavelength Reconfigurable Photonic Switch Using Thermally Tuned Micro-Ring Resonators Fabricated on Silicon Substrate," Frontiers in Optics 2006/Laser Science XXII conference, Rochester, New York. October 8-12, 2006.

Co-author of "A Switched High $T_{c}$ Superconductor Microstrip Resonator Using a MEM Switch," Supercond. Sci. Technol., 2003, vol. 16, pp. 1438-1441.

Co-author of "The Design, Fabrication and Measurement of Tapped Microstrip "T"Resonator Using MEMs Switch High-Tc Superconductor," Advances in Cryogenic Eng., vol. 50, pp. 724-731, 2004.

Co-author of "Laser Ablated and $R F$ Sputtered $\mathrm{BaTiO}_{3}$ Thin Films for Use in a Superconducting RF MEM Switch," Advances in Cryogenic Engineering: Transactions of the International Cryogenic Materials Conf. - ICMC, Vol. 50B, 2004, pp. 747-754.

Co-author of "Elastic modulus study of gold thin film for use as an actuated membrane in a Superconducting RF MEM switch", IEEE Transactions on Applied Superconductivity, vol.15, no.2, pp. 980-983, June 2005.

Presented "MEM Switched High $T_{c}$ Superconductor Tapped Microstrip Resonator" and "Design and Fabrication of a High $\mathrm{T}_{\mathrm{c}}$ Superconducting Coplanar " $\mathrm{T}$ " Resonator with a MEM Shunt Switch for Tuning," CEC/ICMC-2003, September 22-26, 2003, Anchorage, Alaska.

Presented "Design, Simulation, and Fabrication of a MEMS Switched Superconducting Microstrip Hairpin Filter," ASC 2004, October 3-8, Jacksonville, Florida.

Presentation co-author of "Nanophotonics for Communications and Biosensing", Optical Waveguide Sensing \& Imaging in Medicine, Environment, Security and Defence Workshop, NATO-Advanced Study Institute, Gatineau, Québec, Canada, October 12 21, 2006. 